\title{
Pyrrolidine-thiourea as a Bifunctional Organocatalyst: Highly Enantioselective Michael Addition of Cyclohexanone to Nitroolefins
}

\author{
Chun-Li Cao, Meng-Chun Ye, Xiu-Li Sun and Yong Tang * \\ State Key Laboratory of Organometallic Chemistry, Shanghai Institute of Organic Chemistry, 354 Fenglin Road, Shanghai \\ 200032, China. \\ tangy@mail.sioc.ac.cn
}

\section{Supporting Information}

Contents

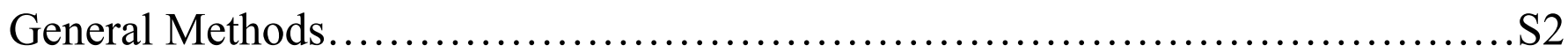

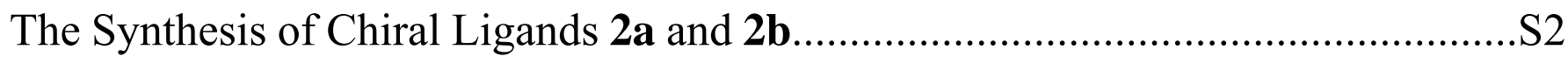

General Procedures for Catalytic Asymmetric Michael Addition........................S3

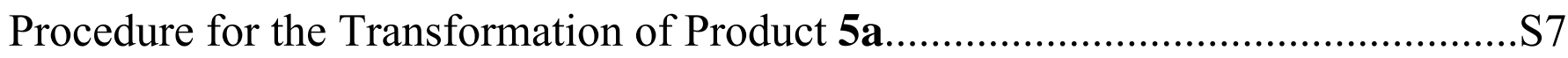

Copies of ${ }^{1} \mathrm{H}$ NMR and ${ }^{13} \mathrm{C}$ NMR of Compounds............................ 8

Copies of HPLC Spectra of Michael Addition Products.......................S32 
General Methods. Commercial Reagents were used as received, unless otherwise noted. All reactions unless otherwise noted were carried out directly under air. Benzene and THF was distilled over calcium hydride. $i$-PrOH, $\mathrm{MeOH}$ and cyclohexanone were distilled before use. Nitroolefins were prepared according to literature procedures. ${ }^{[1,2]}$

Procedures for the preparation of pyrrolidine-urea (thiourea) organocatalysts $2 \mathbf{a}$ and $\mathbf{2 b}$.
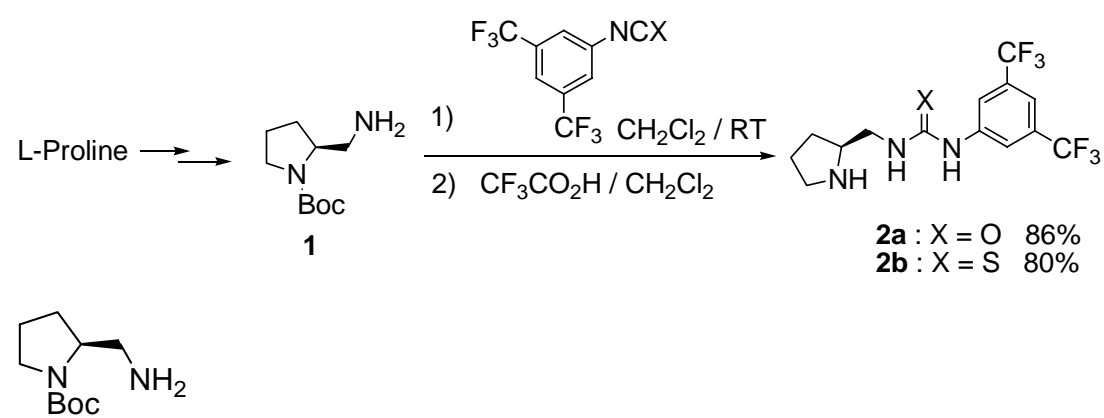

(S)-tert-butyl 2-(aminomethyl)pyrrolidine-1-carboxylate 1. Compound $\mathbf{1}$ is prepared according to the known procedures in 4 steps from L-proline. ${ }^{[3]}$<smiles>O=C(CC1CCCN1)Nc1cc(C(F)(F)F)cc(C(F)(F)F)c1</smiles>

(S)-1-(3,5-bis(trifluoromethyl)phenyl)-3-(pyrrolidin-2-ylmethyl)urea (2a) :

To a stirred solution of $\mathbf{1}(888.6 \mathrm{mg}, 4.35 \mathrm{mmol})$ in dry $\mathrm{CH}_{2} \mathrm{Cl}_{2}(30 \mathrm{~mL})$ was added 3,5-bis(trifluoromethyl)phenyl isocyanate $(0.8 \mathrm{~mL}, 4.35 \mathrm{mmol})$ at room temperature. The reaction mixture was allowed to stir for $12 \mathrm{~h}$. The solvent was removed under reduced pressure to give the crude product $(1.737 \mathrm{~g}, 86 \%)$, which was used directly in the following step without further purification.

The N-Boc-derivative $(145 \mathrm{mg}, 0.32 \mathrm{mmol}$ ) was dissolved in a mixture of trifluoroacetic acid and dichloromethane $(8 \mathrm{~mL}, \mathrm{~V} / \mathrm{V}=1: 1)$ and the solution was stirred for $2 \mathrm{~h}$ at room temperature, at which time the solvent was removed under reduced pressure. The $\mathrm{pH}$ was adjusted to 8 with aqueous $\mathrm{NaHCO}_{3}$. The mixture was extracted with dichloromethane (3 $\times 20 \mathrm{~mL}$ ). The combined organic layers were dried with $\mathrm{Na}_{2} \mathrm{SO}_{4}$. Concentrated and purified by flash column chromatography $(\mathrm{MeOH} / \mathrm{EtOAc}=7 / 1)$ to give thiourea $2 \mathbf{a}$ as a yellow solid $(114 \mathrm{mg}, 99 \%) . \mathrm{Mp} .205^{\circ} \mathrm{C} ;[\alpha]_{\mathrm{D}}{ }^{20}=+4.4(\mathrm{c}$ $\left.=0.885, \mathrm{CHCl}_{3}\right) ;{ }^{1} \mathrm{H}$ NMR $\left(400 \mathrm{MHz}, \mathrm{CD}_{3} \mathrm{OD}\right): \delta 8.07(\mathrm{~s}, 2 \mathrm{H}), 7.47(\mathrm{~s}, 1 \mathrm{H}), 3.80-3.68(\mathrm{~m}, 1 \mathrm{H}), 3.54-3.50(\mathrm{~m}, 2 \mathrm{H}), 3.35-$ $3.27(\mathrm{~m}, 2 \mathrm{H}), 2.16-2.00(\mathrm{~m}, 3 \mathrm{H}), 1.82-1.77(\mathrm{~m}, 1 \mathrm{H}) .{ }^{13} \mathrm{C} \mathrm{NMR}\left(100 \mathrm{MHz}, \mathrm{CD}_{3} \mathrm{OD}\right): 158.8,143.7,133.6(\mathrm{q}, J=32.7 \mathrm{~Hz})$, $125.3(\mathrm{q}, J=270.2 \mathrm{~Hz}), 119.7(\mathrm{~d}, J=3.2 \mathrm{~Hz}), 116.2$ (t, $J=3.8 \mathrm{~Hz}), 63.1,47.1,42.7,28.9,25.0$; IR (neat): 3306, 2970, 1680, 1573, 1388, 1279, 1127, 882, $682 \mathrm{~cm}^{-1}$; MS (ESI, m/z): $356\left(\mathrm{M}+\mathrm{H}^{+}\right)$; HRMS (ESI): Calcd for $\mathrm{C}_{14} \mathrm{H}_{16} \mathrm{~N}_{3} \mathrm{OF}_{6}^{+}$: 356.1190. Found: 356.1192.

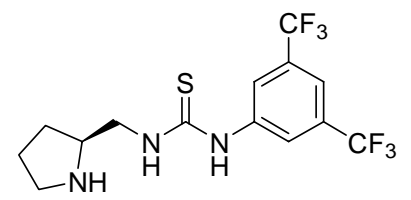

\section{(S)-1-(3,5-bis(trifluoromethyl)phenyl)-3-(pyrrolidin-2-ylmethyl)thiourea 2b :}

yield : 80\%. Mp. $102{ }^{\circ} \mathrm{C} ;[\alpha]_{\mathrm{D}}{ }^{20}=-26.7\left(\mathrm{c}=0.695, \mathrm{CHCl}_{3}\right) ;{ }^{1} \mathrm{H}$ NMR $\left(400 \mathrm{MHz}, \mathrm{CD}_{3} \mathrm{OD}\right): \delta 8.10(\mathrm{~s}, 2 \mathrm{H}), 7.51(\mathrm{~s}, 1 \mathrm{H})$, 3.86-3.78 (m, 3H), 3.25-3.20 (m, 1H), 3.17-3.13 (m, 1H), 2.10-2.00 (m, 1H), 1.97-1.87 (m, 2H), 1.72-1.55 (m, $1 \mathrm{H}) .{ }^{13} \mathrm{C}$ NMR (100 MHz, CD 3 OD): 184.6, 143.2, 133.0 (q, $J=33.2 \mathrm{~Hz}), 125.0$ (q, $J=270.3 \mathrm{~Hz}), 124.5$ (d, $J=3.1 \mathrm{~Hz}), 118.6$ (t, 
$J=3.5 \mathrm{~Hz}$ ), 62.0, 46.9, 46.0, 28.9, 24.7; IR (neat): 3244, 2956, 1605, 1553, 1538, 1473, 1385, 1278, 1131, $682 \mathrm{~cm}^{-1}$; MS (ESI, $m / z): 372\left(\mathrm{M}+\mathrm{H}^{+}\right)$; HRMS (ESI): Calcd for $\mathrm{C}_{14} \mathrm{H}_{16} \mathrm{~N}_{3} \mathrm{SF}_{6}{ }^{+}: 372.0963$. Found: 372.0964.

\section{Typical Procedure for Michael Addition Reactions:}

Procedure A (in solvent): To a solution of catalyst $2 \mathbf{a}$ or $\mathbf{2 b}(0.05 \mathrm{mmol})$ and $n$-butyric acid $(0.0025 \mathrm{mmol})$ in solvent $(1 \mathrm{~mL})$ was added cyclohexanone $(0.25 \mathrm{~mL}, 10 \mathrm{eq})$ at required temperature and the resulting mixture was stirred for 15 min, nitroolefin $(0.25 \mathrm{mmol}, 1 \mathrm{eq})$ was added. After the reaction is complete monitored by TLC, the mixture was concentrated under reduced pressure, the resulting residue was then purified by flash chromatography $(\mathrm{PE}: \mathrm{EtOAc}=1 / 5)$ to give the product.

Procedure B (in neat): The catalyst $\mathbf{2 a}$ or $\mathbf{2 b}(0.05 \mathrm{mmol})$ and n-butyric acid $(0.0025 \mathrm{mmol})$ in cyclohexanone $(0.5 \mathrm{~mL}$, $20 \mathrm{eq})$ at required temperature and the resulting mixture was stirred for $15 \mathrm{~min}$, nitroolefin $(0.25 \mathrm{mmol}, 1 \mathrm{eq})$ was added. After the reaction is complete monitored by TLC, the mixture was concentrated under reduced pressure, the resulting residue was then purified by flash chromatography $(\mathrm{PE}: \mathrm{EtOAc}=1 / 5)$ to give the product.

Relative and absolute configurations of the products were determined by comparison with the known ${ }^{1} \mathrm{H}$ NMR, chiral HPLC analysis, and optical rotation values. Compounds reported in Table 2, entries $\mathbf{1}^{[4]}, \mathbf{2}^{[5]}, \mathbf{3}^{[6]}, \mathbf{6}^{[7]}, \mathbf{8}^{[8]}, \mathbf{9}^{[9]}, \mathbf{1 0}^{[10]}$ are known.<smiles>O=C1CCCCC1C[N+](=O)[O-]</smiles>

(S)-2-((R)-2-nitro-1-phenylethyl)cyclohexanone $\mathbf{5 a}^{[6]}$ : (Procedure B) reaction time: $38 \mathrm{~h}$ (99\% yield); ee was determined by HPLC analysis (Chiralcel AS-H, $i-\mathrm{PrOH} / \mathrm{h}$ exane $=10 / 90,0.7 \mathrm{~mL} / \mathrm{min}, 238 \mathrm{~nm} ; \mathrm{t}_{\mathrm{r}}(\operatorname{minor})=23.88 \mathrm{~min}, \mathrm{t}_{\mathrm{r}}$ $($ major $)=36.38 \mathrm{~min}), 90 \% \mathrm{ee},[\alpha]_{\mathrm{D}}{ }^{20}=-26.4\left(\mathrm{c}=0.945, \mathrm{CHCl}_{3}\right)$; syn/anti $=96 / 4 ;{ }^{1} \mathrm{H} \mathrm{NMR}\left(300 \mathrm{MHz}, \mathrm{CDCl}_{3}\right): \delta 7.34-$ $7.23(\mathrm{~m}, 3 \mathrm{H}), 7.16(\mathrm{~d}, J=6.9 \mathrm{~Hz}, 2 \mathrm{H}), 4.95(\mathrm{dd}, J=4.5,12.6 \mathrm{~Hz}, 1 \mathrm{H}), 4.63(\mathrm{dd}, J=9.9,12.3 \mathrm{~Hz}, 1 \mathrm{H}), 3.76(\mathrm{dt}, J=4.5$, $9.9 \mathrm{~Hz}, 1 \mathrm{H}), 2.68$ (ddd, $J=8.1,8.4,11.7 \mathrm{~Hz}, 1 \mathrm{H}), 2.49-2.33(\mathrm{~m}, 2 \mathrm{H}), 2.10-2.04$ (m, 1H), 1.79-1.52 (m, 4H), 1.52-1.19 $(\mathrm{m}, 1 \mathrm{H})$.<smiles>O=C1CCCCC1CC[N+](=O)[O-]</smiles>

(S)-2-((R)-1-(naphthalen-1-yl)-2-nitroethyl)cyclohexanone $5 \mathbf{b}^{[5]}$ : (Procedure B) reaction time: $60 \mathrm{~h}$ (93\% yield); ee was determined by HPLC analysis (Chiralcel AS, $i-\operatorname{PrOH} /$ hexane $=30 / 70,0.7 \mathrm{~mL} / \mathrm{min}, 238 \mathrm{~nm} ; \mathrm{t}_{\mathrm{r}}$ (minor) $=11.03 \mathrm{~min}$, $\mathrm{t}_{\mathrm{r}}($ major $\left.)=14.45 \mathrm{~min}\right), 95 \%$ ee, $[\alpha]_{\mathrm{D}}{ }^{20}=-101.2\left(\mathrm{c}=1.174, \mathrm{CHCl}_{3}\right) ; \operatorname{syn} / \mathrm{anti}=99 / 1 ;{ }^{1} \mathrm{H} \mathrm{NMR}\left(300 \mathrm{MHz}, \mathrm{CDCl}_{3}\right): \delta 8.15$ (s, 1H), $7.85(\mathrm{~d}, J=8.1 \mathrm{~Hz}, 1 \mathrm{H}), 7.80(\mathrm{~d}, J=8.1 \mathrm{~Hz}, 1 \mathrm{H}), 7.58-7.36(\mathrm{~m}, 4 \mathrm{H}), 5.07$ (dd, $J=4.2,12.6 \mathrm{~Hz}, 1 \mathrm{H}), 4.91(\mathrm{~m}$, 1H), 4.76 (br s, 1H), 2.85 (br s, 1H), 2.52-2.39 (m, 2H), 2.10-2.04 (m, 1H), 1.69-1.47 (m, 4H), 1.30-1.19 (m, 1H).<smiles>O=C1CCCCC1Cc1ccco1</smiles>

(S)-2-((S)-1-(furan-2-yl)-2-nitroethyl)cyclohexanone $5 \mathbf{c}^{[4]}$ : (Procedure B) reaction time: $36 \mathrm{~h}$ (93\% yield); ee was determined by HPLC analysis (Chiralcel AD, $i-\mathrm{PrOH} /$ hexane $=10 / 90,0.7 \mathrm{~mL} / \mathrm{min}, 220 \mathrm{~nm} ; \mathrm{t}_{\mathrm{r}}$ (major) $=15.77 \mathrm{~min}$, $\mathrm{t}_{\mathrm{r}}$ 
$($ minor $)=19.03 \mathrm{~min}), 89 \%$ ee, $[\alpha]_{\mathrm{D}}{ }^{20}=-10.7\left(\mathrm{c}=1.132, \mathrm{CHCl}_{3}\right) ; \operatorname{syn} / \mathrm{anti}=91 / 9 ;{ }^{1} \mathrm{H} \mathrm{NMR}\left(300 \mathrm{MHz}, \mathrm{CDCl}_{3}\right): \delta 7.35$ (m, 1H), $6.28(\mathrm{dd}, J=1.8,3.3 \mathrm{~Hz}, 1 \mathrm{H}), 6.18(\mathrm{~d}, J=3.3 \mathrm{~Hz}, 1 \mathrm{H}), 4.83-4.63(\mathrm{~m}, 2 \mathrm{H}), 3.97(\mathrm{dt}, J=4.8,9.3 \mathrm{~Hz}, 1 \mathrm{H}), 2.80-$ $2.71(\mathrm{~m}, 1 \mathrm{H}), 2.50-2.31(\mathrm{~m}, 2 \mathrm{H}), 2.14-2.04(\mathrm{~m}, 1 \mathrm{H}), 1.88-1.56(\mathrm{~m}, 4 \mathrm{H}), 1.35-1.21(\mathrm{~m}, 1 \mathrm{H})$.<smiles>O=C1CCCCC1C[N+](=O)[O-]</smiles>

(S)-2-((R)-2-nitro-1-(2-nitrophenyl)ethyl)cyclohexanone 5d : (Procedure B) reaction time: $38 \mathrm{~h}$ (95\% yield); ee was determined by HPLC analysis (Chiralcel AD, $i-\mathrm{PrOH} / \mathrm{hexane}=5 / 95,0.9 \mathrm{~mL} / \mathrm{min}, 238 \mathrm{~nm} ; \mathrm{t}_{\mathrm{r}}($ minor $)=51.63 \mathrm{~min}, \mathrm{t}_{\mathrm{r}}$ $($ major $)=63.35 \mathrm{~min}), 97 \% \mathrm{ee},[\alpha]_{\mathrm{D}}{ }^{20}=-153.7\left(\mathrm{c}=1.105, \mathrm{CHCl}_{3}\right) ; \mathrm{syn} / \mathrm{anti}=96 / 4 ;{ }^{1} \mathrm{H} \mathrm{NMR}\left(300 \mathrm{MHz}, \mathrm{CDCl}_{3}\right): \delta 7.83$ (dd, $J=1.2,8.4 \mathrm{~Hz}, 1 \mathrm{H}), 7.64-7.58(\mathrm{~m}, 1 \mathrm{H}), 7.49-7.41(\mathrm{~m}, 2 \mathrm{H}), 4.98-4.83(\mathrm{~m}, 2 \mathrm{H}), 4.38$ (dt, $J=4.2,9.0 \mathrm{~Hz}, 1 \mathrm{H}), 2.98-$ $2.87(\mathrm{~m}, 1 \mathrm{H}), 2.50-2.34(\mathrm{~m}, 2 \mathrm{H}), 2.17-2.07(\mathrm{~m}, 1 \mathrm{H}), 1.85-1.61(\mathrm{~m}, 5 \mathrm{H}) .{ }^{13} \mathrm{C}$ NMR $\left(75 \mathrm{MHz}, \mathrm{CDCl}_{3}\right)$ :211.1, 150.7, 133.1, 132.8, 129.1, 128.6, 124.9, 77.6, 52.1, 42.8, 38.6, 33.2, 28.3, 25.3; IR (film): 2944, 2864, 1707, 1552, 1527, 1358, 855, $781 \mathrm{~cm}^{-1}$; MS (ESI, m/z): $310\left(\mathrm{M}+\mathrm{NH}_{4}{ }^{+}\right.$); HRMS (ESI): Calcd for $\mathrm{C}_{14} \mathrm{H}_{16} \mathrm{~N}_{2} \mathrm{O}_{5} \mathrm{Na}^{+}: 315.0960$. Found: 315.0951 .

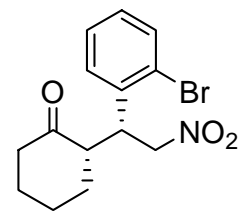

(S)-2-((R)-1-(2-bromophenyl)-2-nitroethyl)cyclohexanone 5e : (Procedure B) reaction time: 29 h (88\% yield); ee was determined by HPLC analysis (Chiralcel AS, $i-\mathrm{PrOH} /$ hexane $=10 / 90,0.8 \mathrm{~mL} / \mathrm{min}, 238 \mathrm{~nm} ; \mathrm{t}_{\mathrm{r}}$ (minor) $=16.64 \mathrm{~min}$, $\mathrm{t}_{\mathrm{r}}($ major $\left.)=20.81 \mathrm{~min}\right), 96 \% \mathrm{ee},[\alpha]_{\mathrm{D}}{ }^{20}=-52.9\left(\mathrm{c}=1.610, \mathrm{CHCl}_{3}\right) ; \mathrm{syn} / \mathrm{anti}=99 / 1 ;{ }^{1} \mathrm{H} \mathrm{NMR}\left(300 \mathrm{MHz}, \mathrm{CDCl}_{3}\right): \delta 7.47$ $(\mathrm{dd}, J=1.2,8.1 \mathrm{~Hz}, 1 \mathrm{H}), 7.23-7.13(\mathrm{~m}, 2 \mathrm{H}), 7.06-7.00(\mathrm{~m}, 1 \mathrm{H}), 4.87-4.73(\mathrm{~m}, 2 \mathrm{H}), 4.30-4.22(\mathrm{~m}, 1 \mathrm{H}), 2.78(\mathrm{~m}, 1 \mathrm{H})$, 2.38-2.28 (m, 2H), 2.03-1.97 (m, 1H), 1.72-1.45 (m, 4H), 1.30-1.26 (m, 1H). ${ }^{13} \mathrm{C}$ NMR (75 MHz, DMSO-d 6 ): 211.0, 137.5, 132.5, 128.7, 128.4, 127.9, 125.0, 77.7, 51.3, 41.8, 41.2, 31.7, 27.7, 24.2; IR (neat): 2941, 2862, 1707, 1551, 1379, 1024, $753 \mathrm{~cm}^{-1}$; MS (ESI, m/z): $327\left(\mathrm{M}+\mathrm{H}^{+}\right)$; Anal. Calcd for $\mathrm{C}_{14} \mathrm{H}_{16} \mathrm{BrNO}_{3}: \mathrm{C}, 51.55 ; \mathrm{H}, 4.94 ; \mathrm{N}, 4.29$. Found: $\mathrm{C}$, $51.72 ; \mathrm{H}, 5.01 ; \mathrm{N}, 4.11$.<smiles>O=C1CCCCC1C[N+](=O)[O-]</smiles>

(S)-2-((R)-1-(4-bromophenyl)-2-nitroethyl)cyclohexanone 5f ${ }^{[7]}$ : (Procedure B) reaction time: $38 \mathrm{~h}$ (90\% yield); ee was determined by HPLC analysis (Chiralcel AS, $i-\mathrm{PrOH} /$ hexane $=10 / 90,1.0 \mathrm{~mL} / \mathrm{min}, 238 \mathrm{~nm}$; $\mathrm{t}_{\mathrm{r}}$ (minor) $=15.52 \mathrm{~min}$, $\mathrm{t}_{\mathrm{r}}($ major $\left.)=22.95 \mathrm{~min}\right), 95 \% \mathrm{ee},[\alpha]_{\mathrm{D}}{ }^{20}=-22.6\left(\mathrm{c}=1.296, \mathrm{CHCl}_{3}\right) ; \mathrm{syn} / \mathrm{anti}=95 / 5 ;{ }^{1} \mathrm{H} \mathrm{NMR}\left(300 \mathrm{MHz}, \mathrm{CDCl}_{3}\right): \delta 7.46$ $(\mathrm{d}, J=8.7 \mathrm{~Hz}, 2 \mathrm{H}), 7.06$ (d, $J=8.7 \mathrm{~Hz}, 2 \mathrm{H}), 4.93(\mathrm{dd}, J=4.5,12.6 \mathrm{~Hz}, 1 \mathrm{H}), 4.60(\mathrm{dd}, J=10.2,12.6 \mathrm{~Hz}, 1 \mathrm{H}), 3.75(\mathrm{dt}, J$ $=4.5,9.9 \mathrm{~Hz}, 1 \mathrm{H}), 2.69-2.60(\mathrm{~m}, 1 \mathrm{H}), 2.51-2.32(\mathrm{~m}, 2 \mathrm{H}), 2.15-2.05(\mathrm{~m}, 1 \mathrm{H}), 1.85-1.58(\mathrm{~m}, 4 \mathrm{H}), 1.30-1.16(\mathrm{~m}, 1 \mathrm{H})$. 
<smiles>O=C1CCCCC1C[N+](=O)[O-]</smiles>

(S)-2-((R)-1-(2,4-dichlorophenyl)-2-nitroethyl)cyclohexanone 5g : (Procedure B) reaction time: 49 h (89\% yield); ee was determined by HPLC analysis (Chiralcel AS, $i-\mathrm{PrOH} /$ hexane $=10 / 90,0.8 \mathrm{~mL} / \mathrm{min}, 238 \mathrm{~nm}$; $\mathrm{t}_{\mathrm{r}}$ (minor) $=12.89 \mathrm{~min}$, $\mathrm{t}_{\mathrm{r}}($ major $\left.)=19.00 \mathrm{~min}\right), 97 \% \mathrm{ee},[\alpha]_{\mathrm{D}}{ }^{20}=-50.9(\mathrm{c}=0.990, \mathrm{CHCl}) ;$ syn/anti $=97 / 3 ;{ }^{1} \mathrm{H} \mathrm{NMR}\left(300 \mathrm{MHz}, \mathrm{CDCl}_{3}\right): \delta 7.40$ $(\mathrm{d}, J=1.8 \mathrm{~Hz}, 1 \mathrm{H}), 7.24$ (dd, $J=2.1,8.7 \mathrm{~Hz}, 2 \mathrm{H}), 4.95-4.80(\mathrm{~m}, 2 \mathrm{H}), 4.27(\mathrm{dt}, J=4.8,9.6 \mathrm{~Hz}, 1 \mathrm{H}), 2.90-2.81(\mathrm{~m}, 1 \mathrm{H})$, 2.48-2.32 (m, 2H), 2.14-2.05 (m, 1H), 1.84-1.52 (m, 4H), 1.40-1.26 (m, 1H). ${ }^{13} \mathrm{C}$ NMR (75 MHz, $\left.\mathrm{CDCl}_{3}\right): 211.2,135.1$, 134.1, 133.8, 130.0, 127.6, 76.9, 51.5, 42.6, 40.3, 32.8, 28.3, 25.1; IR (neat): 2942, 2863, 1708, 1552, 1475, 1379, 820, $733 \mathrm{~cm}^{-1}$; MS (ESI, m/z): $316\left(\mathrm{M}+\mathrm{H}^{+}\right)$; HRMS (ESI): Calcd for $\mathrm{C}_{14} \mathrm{H}_{15} \mathrm{NO}_{3} \mathrm{Cl}_{2} \mathrm{Na}^{+}: 338.0330$. Found: 338.0321 .<smiles>O=C1CCCCC1CC[N+](=O)[O-]</smiles>

(S)-2-((R)-1-(benzo[d][1,3]dioxol-5-yl)-2-nitroethyl)cyclohexanone $5 \mathbf{h}^{[8]}$ : (Procedure B) reaction time: $38 \mathrm{~h}(87 \%$ yield); ee was determined by HPLC analysis (Chiralcel AD-H, $i-\mathrm{PrOH} / \mathrm{hexane}=5 / 95,0.7 \mathrm{~mL} / \mathrm{min}, 214 \mathrm{~nm}$; $\mathrm{t}_{\mathrm{r}}$ (minor) = $48.00 \mathrm{~min}, \mathrm{t}_{\mathrm{r}}$ (major) $\left.=52.13 \mathrm{~min}\right), 98 \%$ ee, $[\alpha]_{\mathrm{D}}{ }^{20}=-25.6\left(\mathrm{c}=0.776, \mathrm{CHCl}_{3}\right) ;$ syn $/$ anti $=94 / 6 ;{ }^{1} \mathrm{H} \mathrm{NMR}(300 \mathrm{MHz}$, $\left.\mathrm{CDCl}_{3}\right): \delta 6.74(\mathrm{~d}, J=7.8 \mathrm{~Hz}, 1 \mathrm{H}), 6.64(\mathrm{~s}, 1 \mathrm{H}), 6.62(\mathrm{~d}, J=7.8 \mathrm{~Hz}, 1 \mathrm{H}), 5.96(\mathrm{~s}, 2 \mathrm{H}), 4.90(\mathrm{dd}, J=4.5,12.3 \mathrm{~Hz}, 1 \mathrm{H})$, $4.55(\mathrm{dd}, J=10.2,12.0 \mathrm{~Hz}, 1 \mathrm{H}), 3.68(\mathrm{dt}, J=4.2,9.9 \mathrm{~Hz}, 1 \mathrm{H}), 2.65-2.56(\mathrm{~m}, 1 \mathrm{H}), 2.52-2.33(\mathrm{~m}, 2 \mathrm{H}), 2.15-2.05(\mathrm{~m}, 1 \mathrm{H})$, $1.83-1.59(\mathrm{~m}, 4 \mathrm{H}), 1.31-1.18(\mathrm{~m}, 1 \mathrm{H})$.<smiles>COc1ccc(C(C[N+](=O)[O-])C2CCCCC2=O)cc1</smiles>

(S)-2-((R)-1-(4-methoxyphenyl)-2-nitroethyl)cyclohexanone 5i ${ }^{[9]}$ : (Procedure B) reaction time: $44 \mathrm{~h}$ (95\% yield); ee was determined by HPLC analysis (Chiralcel AD-H, $i-P r O H / h e x a n e=25 / 75,0.7 \mathrm{~mL} / \mathrm{min}, 238 \mathrm{~nm} ; \mathrm{t}_{\mathrm{r}}$ (minor) $=13.46$

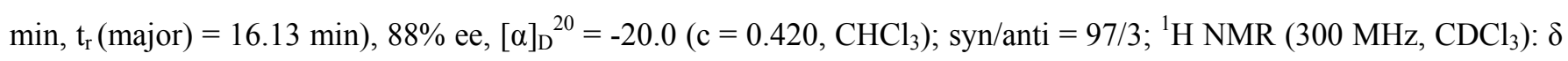
$7.08(\mathrm{~d}, J=8.7 \mathrm{~Hz}, 2 \mathrm{H}), 6.85(\mathrm{~d}, J=8.7 \mathrm{~Hz}, 2 \mathrm{H}), 4.91(\mathrm{dd}, J=4.5,12.3 \mathrm{~Hz}, 1 \mathrm{H}), 4.58(\mathrm{dd}, J=9.9,12.0 \mathrm{~Hz}, 1 \mathrm{H}), 3.78$ (s, $3 \mathrm{H}), 3.71(\mathrm{dt}, J=4.5,9.9 \mathrm{~Hz}, 1 \mathrm{H}), 2.69-2.60(\mathrm{~m}, 1 \mathrm{H}), 2.52-2.32(\mathrm{~m}, 2 \mathrm{H}), 2.13-2.03(\mathrm{~m}, 1 \mathrm{H}), 1.83-1.51(\mathrm{~m}, 4 \mathrm{H}), 1.30-$ $1.16(\mathrm{~m}, 1 \mathrm{H})$.<smiles>CC(C)C(C[N+](=O)[O-])C1CCCCC1=O</smiles>

(S)-2-((S)-3-methyl-1-nitrobutan-2-yl)cyclohexanone 5j ${ }^{[10]}$ : (Procedure B) reaction time: $6 \mathrm{~d}$ (63\% yield); ee was determined by HPLC analysis (Chiralcel AD-H, $i-\mathrm{PrOH} /$ hexane $=10 / 90,1.0 \mathrm{~mL} / \mathrm{min}, 214 \mathrm{~nm} ; \mathrm{t}_{\mathrm{r}}$ (major) $=6.31 \mathrm{~min}$, $\mathrm{t}_{\mathrm{r}}$ $($ minor $)=7.76 \mathrm{~min}), 94 \% \mathrm{ee},[\alpha]_{\mathrm{D}}{ }^{20}=-28.5\left(\mathrm{c}=1.305, \mathrm{CHCl}_{3}\right)$; syn/anti $=99 / 1 ;{ }^{1} \mathrm{H} \mathrm{NMR}\left(300 \mathrm{MHz}, \mathrm{CDCl}_{3}\right): \delta 4.64(\mathrm{dd}$, $J=5.7,13.8 \mathrm{~Hz}, 1 \mathrm{H}), 4.35(\mathrm{dd}, J=5.1,13.8 \mathrm{~Hz}, 1 \mathrm{H}), 2.67-2.60(\mathrm{~m}, 1 \mathrm{H}), 2.43-2.28(\mathrm{~m}, 3 \mathrm{H}), 2.10(\mathrm{~m}, 2 \mathrm{H}), 1.99-1.88(\mathrm{~m}$, 
2H), 1.74-1.54 (m, 3H), $0.94(\mathrm{~d}, J=6.6 \mathrm{~Hz}, 3 \mathrm{H}), 0.90(\mathrm{~d}, J=6.6 \mathrm{~Hz}, 3 \mathrm{H})$.<smiles>O=C1CCCC1C[N+](=O)[O-]</smiles>

(S)-2-((R)-2-nitro-1-phenylethyl)cyclopentanone 5l ${ }^{[11]}$ : (Procedure B) reaction time: $6 \mathrm{~d}$ (27\% yield); ee was determined by HPLC analysis (Chiralcel AD, $i-\mathrm{PrOH} / \mathrm{hexane}=5 / 95,1.0 \mathrm{~mL} / \mathrm{min}, 220 \mathrm{~nm} ; \mathrm{t}_{\mathrm{r}}(\operatorname{minor})=14.42 \mathrm{~min}$, $\mathrm{t}_{\mathrm{r}}$ $($ major $)=20.05 \mathrm{~min}), 71 \%$ ee, $[\alpha]_{\mathrm{D}}{ }^{20}=-15.5\left(\mathrm{c}=0.495, \mathrm{CHCl}_{3}\right) ;$ syn $/ \mathrm{anti}=75 / 15 ;{ }^{1} \mathrm{H} \mathrm{NMR}\left(300 \mathrm{MHz}, \mathrm{CDCl}_{3}\right): \delta$ 7.34-7.23 (m, 3H), 7.20-7.15 (m, 2H), 5.37-5.30 (m, 1H), $4.71(\mathrm{dd}, J=10.2,12.6 \mathrm{~Hz}, 1 \mathrm{H}), 3.76-3.65(\mathrm{~m}, 1 \mathrm{H}), 2.44-2.31$ $(\mathrm{m}, 2 \mathrm{H}), 2.19-2.06(\mathrm{~m}, 1 \mathrm{H}), 1.94-1.83(\mathrm{~m}, 2 \mathrm{H}), 1.76-1.66(\mathrm{~m}, 1 \mathrm{H}), 1.55-1.41(\mathrm{~m}, 1 \mathrm{H})$.<smiles>CC(=O)CC(C[N+](=O)[O-])c1ccccc1</smiles>

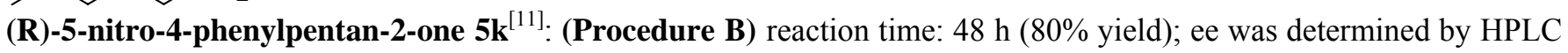
analysis $\left(\right.$ Chiralcel AS, $i-\mathrm{PrOH} /$ hexane $=15 / 85,1.0 \mathrm{~mL} / \mathrm{min}, 220 \mathrm{~nm} ; \mathrm{t}_{\mathrm{r}}$ (minor) $=18.85 \mathrm{~min}, \mathrm{t}_{\mathrm{r}}$ (major) $=22.13 \mathrm{~min}$, $48 \%$ ee; ${ }^{1} \mathrm{H}$ NMR $\left(300 \mathrm{MHz}, \mathrm{CDCl}_{3}\right): \delta$ 7.28-7.13 (m, 5H), 4.67-4.49 (m, 2H), 3.98-3.89 (m, 1H), $2.84(\mathrm{~d}, J=6.9 \mathrm{~Hz}$, $2 \mathrm{H}), 2.05(\mathrm{~s}, 3 \mathrm{H})$.<smiles>CC(=O)C(C)C[N+](=O)[O-]</smiles>

(3S,4R)-3-methyl-5-nitro-4-phenylpentan-2-one $5 \mathrm{ma}^{[11]}$ : (Procedure B) reaction time: $48 \mathrm{~h}(90 \%$ total yield for compound 5ma and 5mb); ee was determined by HPLC analysis (Chiralcel AD-H, i-PrOH/hexane = 1.2/100, 0.6 $\mathrm{mL} / \mathrm{min}, 238 \mathrm{~nm} ; \mathrm{t}_{\mathrm{r}}($ minor $)=43.45 \mathrm{~min}, \mathrm{t}_{\mathrm{r}}($ major $\left.)=54.10 \mathrm{~min}\right), 70 \%$ ee, $[\alpha]_{\mathrm{D}}{ }^{20}=-10.6\left(\mathrm{c}=1.250, \mathrm{CHCl}_{3}\right) ; \operatorname{syn} /$ anti $=$ 99/1; ${ }^{1} \mathrm{H}$ NMR (300 MHz, $\left.\mathrm{CDCl}_{3}\right): \delta$ 7.37-7.30 (m, 3H), 7.18-7.15 (m, 2H), 4.67-4.63 (m, 2H), 3.80 (dt, J = 5.7, 9.0 Hz, $1 \mathrm{H}), 3.03-2.93(\mathrm{~m}, 1 \mathrm{H}), 2.24(\mathrm{~s}, 3 \mathrm{H}), 0.98(\mathrm{~d}, J=7.2 \mathrm{~Hz}, 3 \mathrm{H})$.<smiles>CCC(=O)CC(C[N+](=O)[O-])c1ccccc1</smiles>

(R)-6-nitro-5-phenylhexan-3-one $\mathbf{5} \mathbf{m b}^{[11]}$ : (Procedure B) reaction time: $48 \mathrm{~h}(90 \%$ total yield for compound $\mathbf{5 m a}$ and $5 \mathrm{mb}$ ); ee was determined by HPLC analysis (Chiralcel AD-H, $i-\mathrm{PrOH} /$ hexane $=1.2 / 100,0.6 \mathrm{~mL} / \mathrm{min}, 238 \mathrm{~nm}$; $\mathrm{t}_{\mathrm{r}}$ (minor) $=60.36 \mathrm{~min}, \mathrm{t}_{\mathrm{r}}$ (major) $\left.=65.47 \mathrm{~min}\right), 70 \%$ ee; $[\alpha]_{\mathrm{D}}{ }^{20}=-6.6\left(\mathrm{c}=0.735, \mathrm{CHCl}_{3}\right) ;{ }^{1} \mathrm{H} \mathrm{NMR}\left(300 \mathrm{MHz}, \mathrm{CDCl}_{3}\right): \delta 7.33-7.26$ (m, 3H), 7.23-7.20 (m, 2H), 4.74-4.54 (m, 2H), 4.08-3.98 (m, 1H), 2.88 (d, J = 7.2 Hz, 2H), 2.43-2.33 (m, 2H), $1.00(\mathrm{t}, J$ $=7.2 \mathrm{~Hz}, 3 \mathrm{H})$.<smiles>CC(C)(C)C(CO)c1ccccc1</smiles>

(R)-2,2-dimethyl-4-nitro-3-phenylbutanal 5n ${ }^{[12]}$ : (Procedure A) reaction time: $48 \mathrm{~h}$ (62\% yield); ee was determined by HPLC analysis (Chiralcel AS-H, $i-P r O H / h e x a n e=10 / 90,0.7 \mathrm{~mL} / \mathrm{min}, 238 \mathrm{~nm}$; $\mathrm{t}_{\mathrm{r}}\left(\right.$ minor) $=20.38 \mathrm{~min}, \mathrm{t}_{\mathrm{r}}$ $($ major $)=21.96 \mathrm{~min}), 82 \% \mathrm{ee},[\alpha]_{\mathrm{D}}{ }^{20}=+0.3\left(\mathrm{c}=0.625, \mathrm{CHCl}_{3}\right) ;{ }^{1} \mathrm{H}$ NMR $\left(300 \mathrm{MHz}, \mathrm{CDCl}_{3}\right): \delta 9.53(\mathrm{~s}, 1 \mathrm{H}), 7.36-7.30$ (m, 3H), 7.22-7.18 (m, 2H), $4.86(\mathrm{dd}, J=11.4,12.9 \mathrm{~Hz}, 1 \mathrm{H}), 4.69$ (dd, $J=3.9,12.6 \mathrm{~Hz}, 1 \mathrm{H}), 3.79(\mathrm{dd}, J=4.2,11.1 \mathrm{~Hz}$, 
1H), $1.14(\mathrm{~s}, 3 \mathrm{H}), 1.01(\mathrm{~s}, 3 \mathrm{H})$.

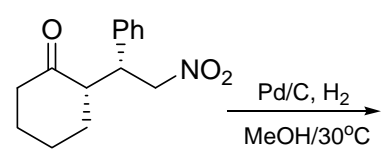

5a $90 \%$ ee

O<smiles>O=C1CCCC[C@@H]1c1ccccc1</smiles>

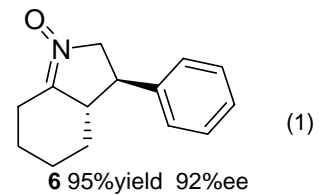

(1)

(3R,3aS)-3-phenyl-3,3a,4,5,6,7-hexahydro-2H-indole 1-oxide $7^{[13]}$ : A suspension of Pd/C (8 mg) and 5a (50 $\mathrm{mg})$ in $\mathrm{MeOH}(5 \mathrm{~mL})$ was stirred at room temperature under $3 \mathrm{~atm}$ hydrogen atmosphere. After being stirred for $12 \mathrm{~h}$, the mixture was filtrated through a pad of Celite and the filtration was concentrated in vacuo, the residue was purified by column chromatography on silica gel to afford desired product 7 (43 $\mathrm{mg}, 95 \%$ yield): ee was determined by HPLC analysis $\left(\right.$ Chiralcel AD-H, $i-\mathrm{PrOH} /$ hexane $=10 / 90,0.6 \mathrm{~mL} / \mathrm{min}, 238 \mathrm{~nm}$; $\mathrm{t}_{\mathrm{r}}$ (major) $=18.02 \mathrm{~min}, \mathrm{t}_{\mathrm{r}}($ minor $\left.)=22.83 \mathrm{~min}\right)$, $92 \%$ ee. Mp. $86^{\circ} \mathrm{C} ;[\alpha]_{\mathrm{D}}{ }^{20}=-34.1\left(\mathrm{c}=1.140, \mathrm{CHCl}_{3}\right) ; \operatorname{syn} /$ anti $>99 / 1 ;{ }^{1} \mathrm{H}$ NMR $\left(300 \mathrm{MHz}, \mathrm{CDCl}_{3}\right): \delta 7.30-7.25(\mathrm{~m}, 2 \mathrm{H})$, 7.21-7.15 (m, 3H), 4.23-4.04 (m, 2H), 3.19-3.01 (m, 2H), $2.73(\mathrm{~m}, 1 \mathrm{H}), 2.05-1.87(\mathrm{~m}, 3 \mathrm{H}), 1.77(\mathrm{~m}, 1 \mathrm{H}), 1.33-1.14(\mathrm{~m}$, 3H). ${ }^{13} \mathrm{C}$ NMR (75 MHz, $\left.\mathrm{CDCl}_{3}\right): 147.9,139.4,128.6,127.0,126.9,67.9,50.2,45.5,32.0,23.9,23.4,23.1$; IR (neat): 2934, 2857, 1621, 1447, 1253, 1231, 1179, 765, $702 \mathrm{~cm}^{-1}$; MS (ESI, $\left.\mathrm{m} / \mathrm{z}\right): 216\left(\mathrm{M}+\mathrm{H}^{+}\right)$; HRMS (ESI): Calcd for $\mathrm{C}_{14} \mathrm{H}_{17} \mathrm{NONa}^{+}: 238.1204$. Found: 238.1202.

[1] For the preparation of aryl nitroolefins: Jang, Y.-J.; Shih, Y.-K.; Liu, J.-Y.; Kuo, W.-Y.; Yao, C.-F. Chem. Europ. J. 2003, 9, 2123-2128.

[2] For the preparation of alkyl nitroolefines: Kumaran, G.; Kulkarni, G. H. Synthesis 1995, 1545-1548.

[3] Dahlin, N.; Boegevig, A.; Adolfsson, H. Adv. Synth. Catal. 2004, 346, 1101-1105.

[4] Alexakis, A.; Andrey, O. Org. Lett. 2002, 4, 3611-3614.

[5] Ishii, T.; Fujioka, S.; Sekiguchi, Y.; Kotsuki, H. J. Am. Chem. Soc. 2004, 126, 9558-9559.

[6] Cobb, A. J. A.; Longbottom, D. A.; Shaw, D. M.; Ley, S. V. Chem. Commun. 2004, 1808-1809.

[7] Risaliti, A. Tetrahedron Lett. 1966, 51, 6331-6335.

[8] Sanchez, I. H.; Larraza, M. I.; Rojas, I.; Brena, F. K.; Flores, H. J. Heterocycles 1985, 23, 3033-3039.

[9] Betancort, J. M.; Sakthivel, K.; Thayumanavan, R.; Tanaka, F.; Barbas, C. F. Synthesis 2004, 1509-1521.

[10] Seebach, D.; Golinski, J. Helv. Chim. Acta. 1981, 64, 1413-1423.

[11] Andrey, O. Alexakis, A. Tomassini, A. Bernardinelli, G. Adv. Synth. Catal. 2004, 346, 1147-1168.

[12] Wang, W. Wang, J. Li, H. Angew. Chem. Int. Ed. 2005, 44, 1369-1371.

[13] Okino, T.; Hoashi, Y.; Furukawa, T.; Xu, X.-N.; Takemoto, Y. J. Am. Chem. Soc. 2005, 127, 119-125. 
1

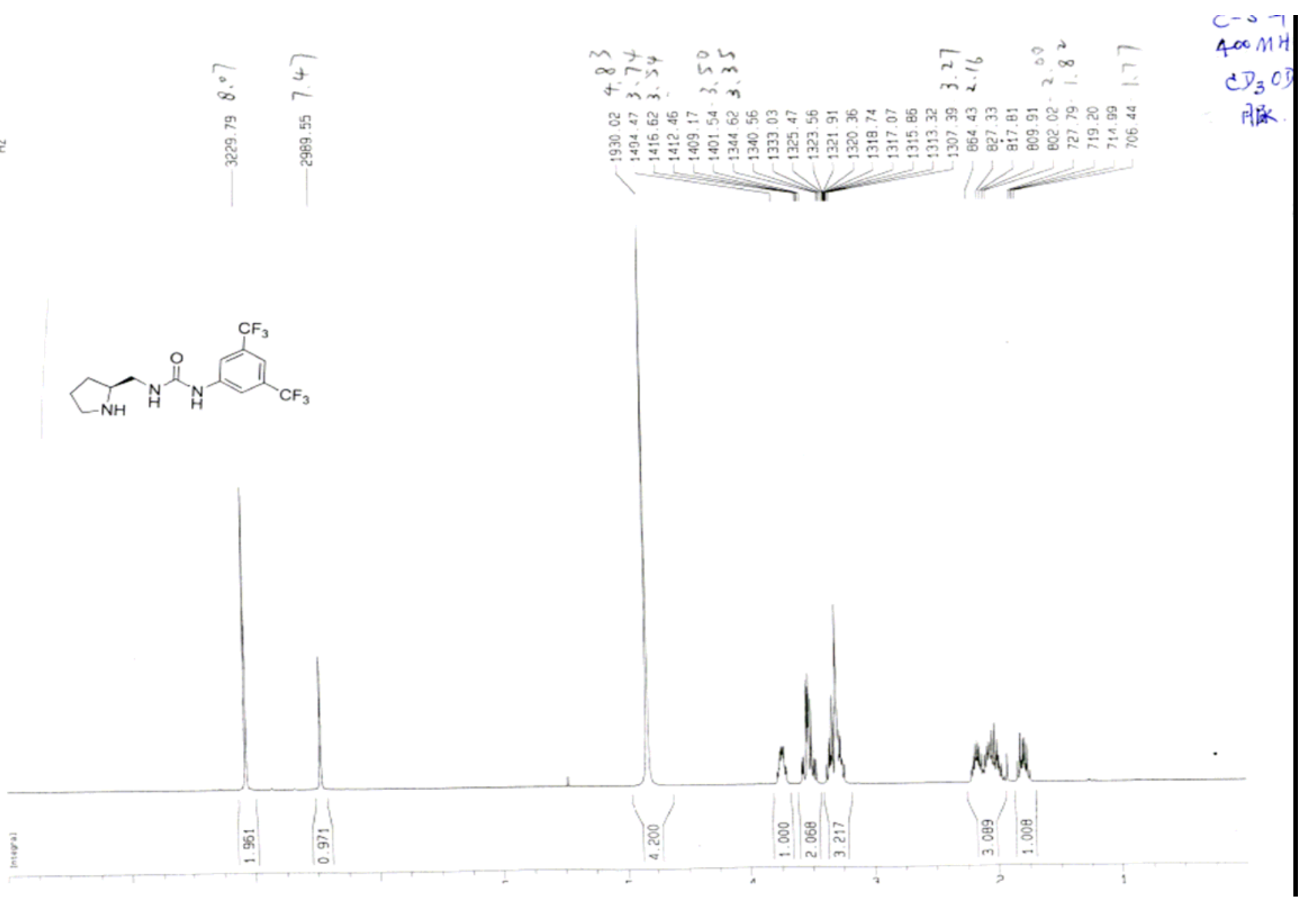




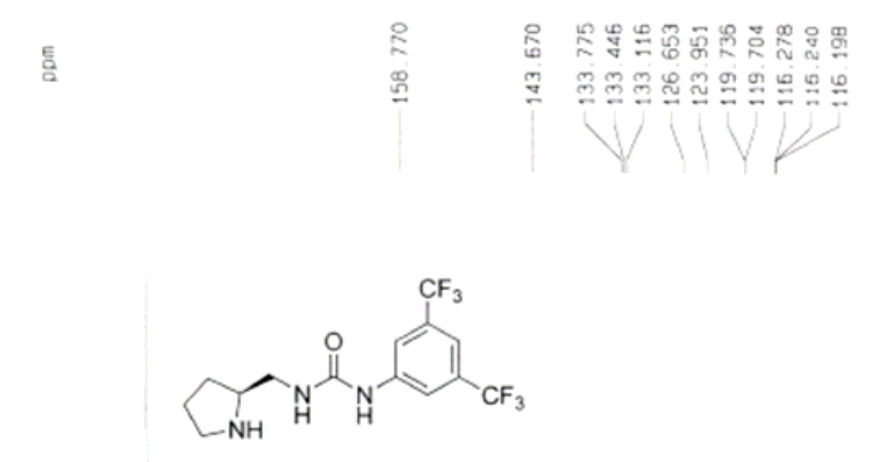

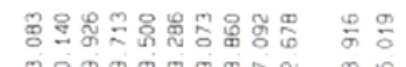

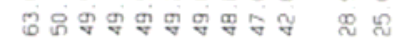

$400 M H=$
$C-5-1$
$C D_{3}$ O 1
Ange

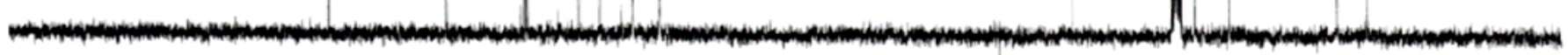

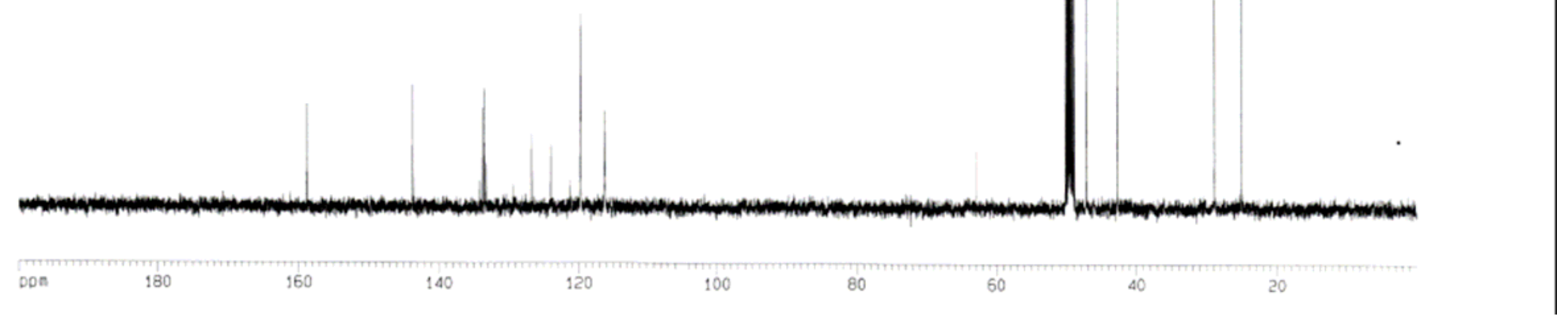




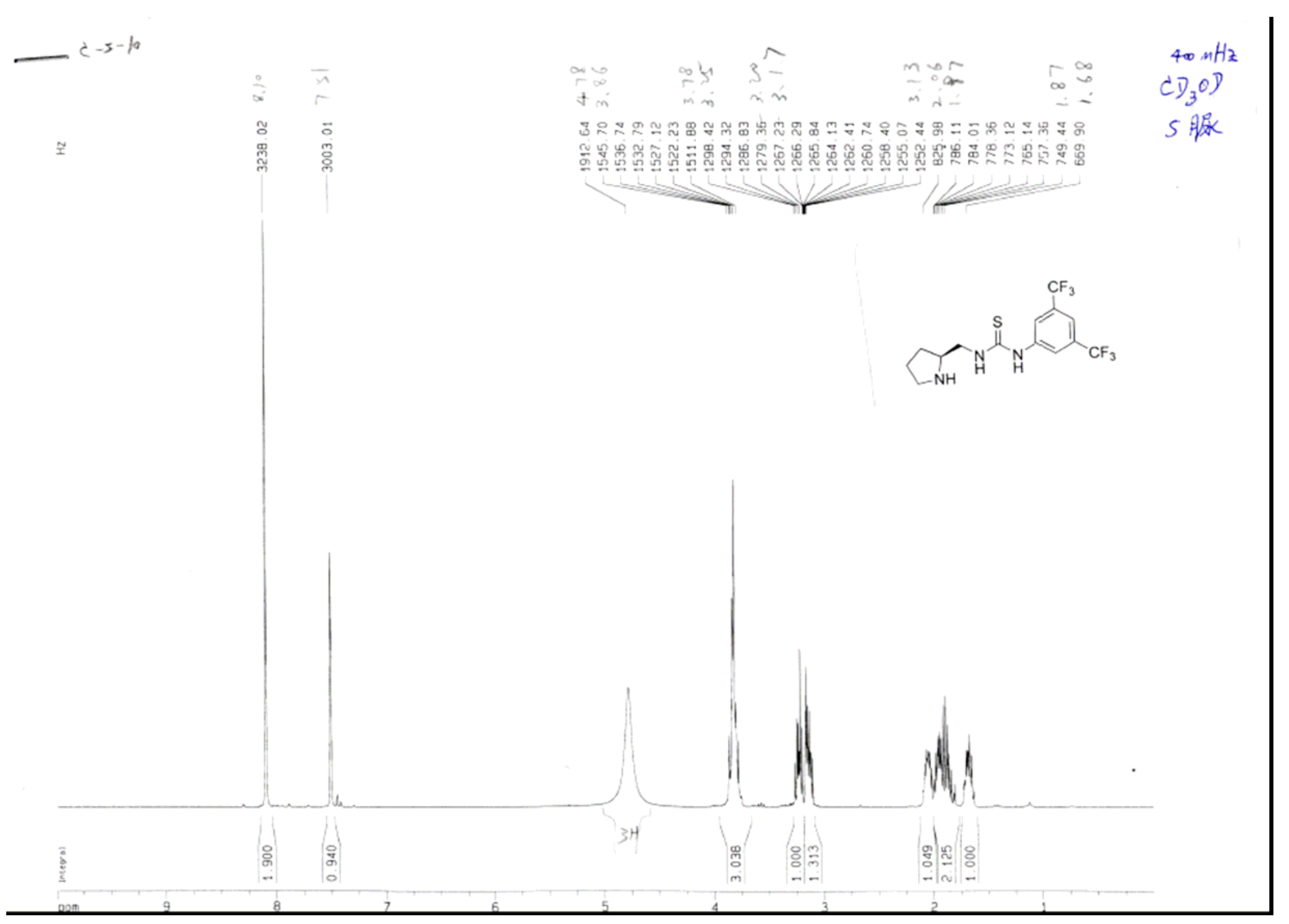




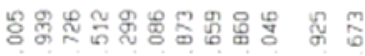

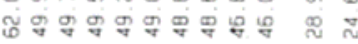

$\mathrm{NH}_{\mathrm{NH}}$

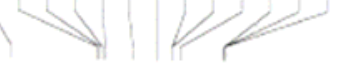




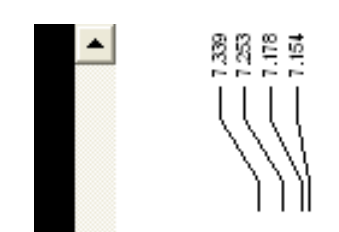

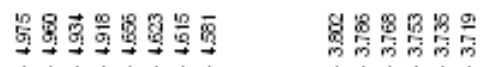

(iv)

vivij

mililifili
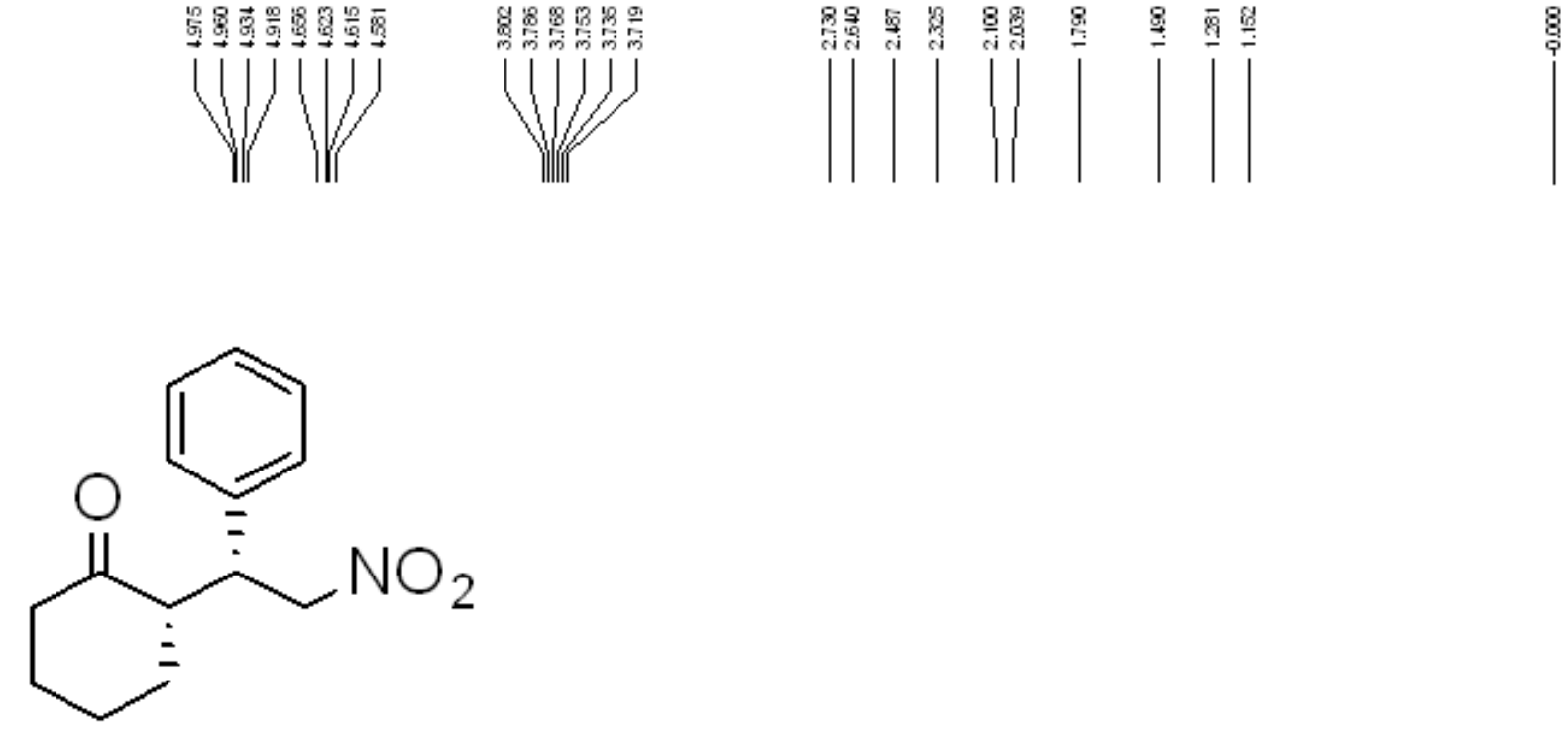

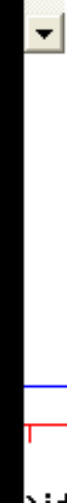
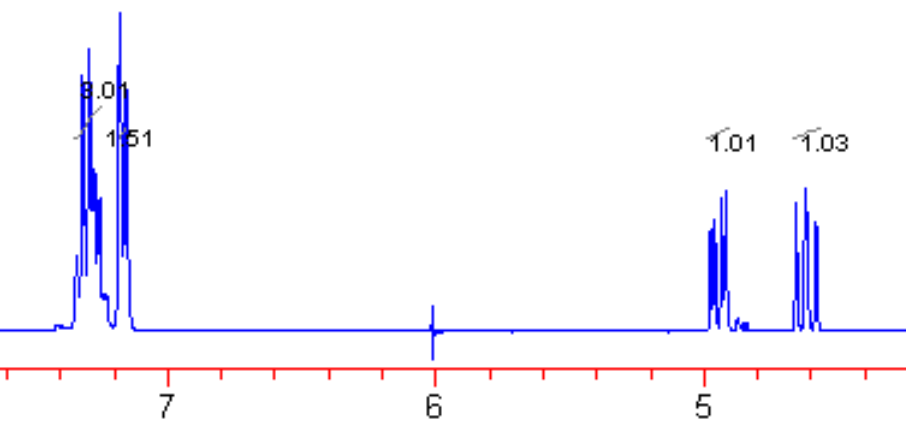

$-1.00$

$0.99^{-2.35}-1.16 \int^{4.42} 1.25$

III

what 

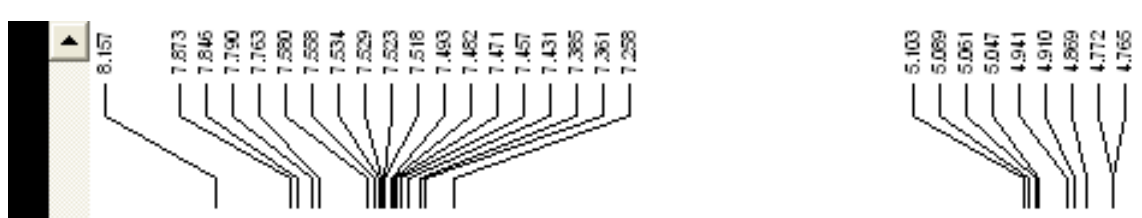

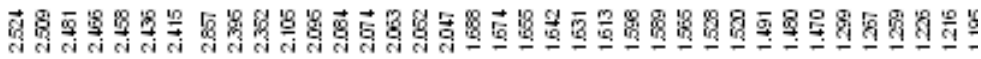

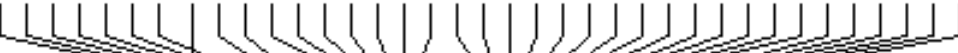

$+\infty$<smiles>O=C1CCCC[C@H]1[C@H](C[N+](=O)[O-])c1cccc2ccccc12</smiles>

$\checkmark$

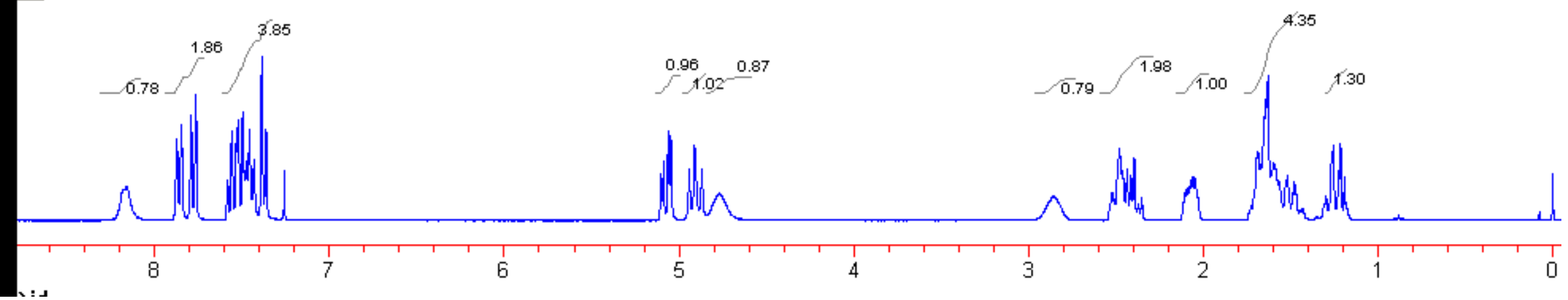




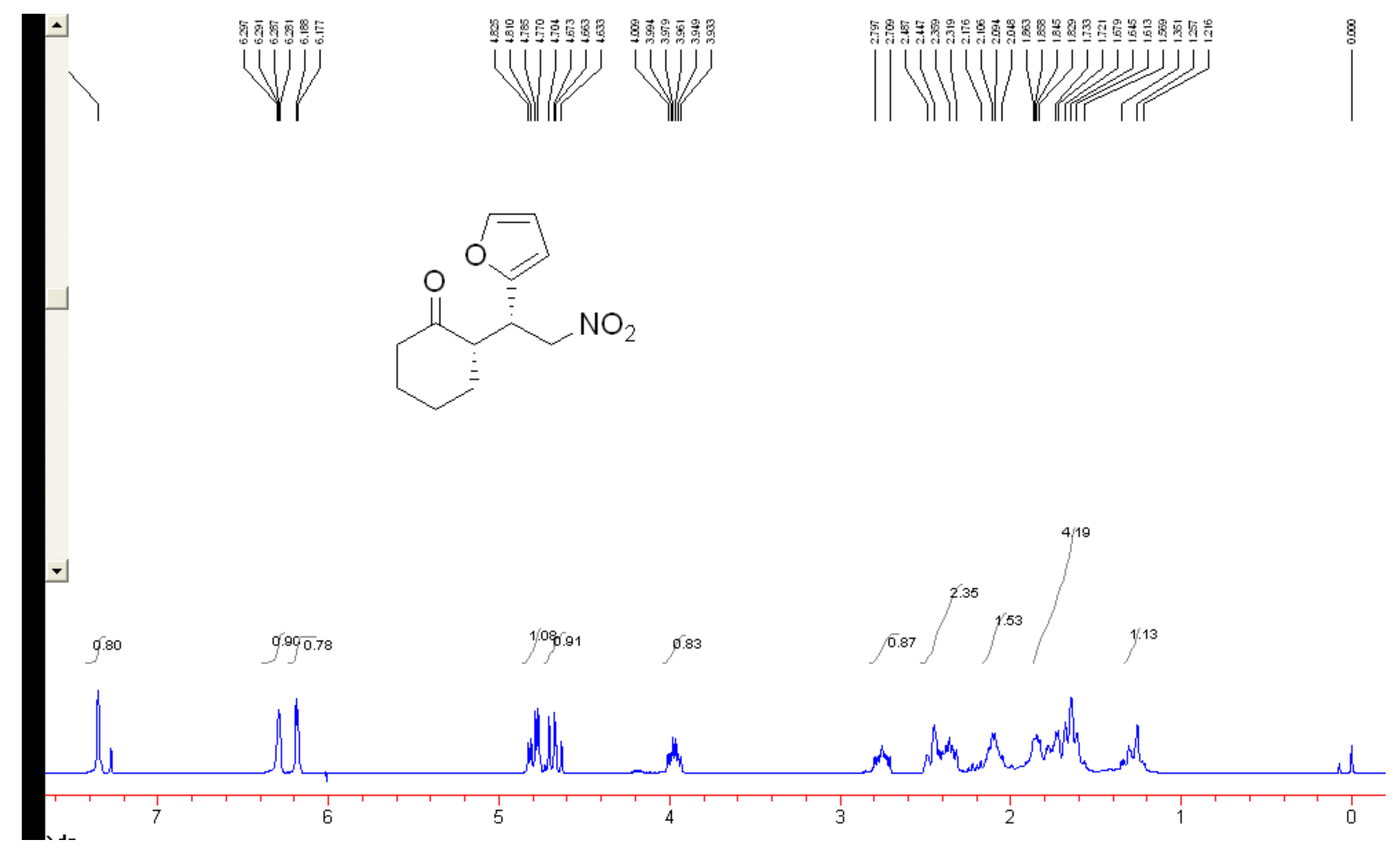



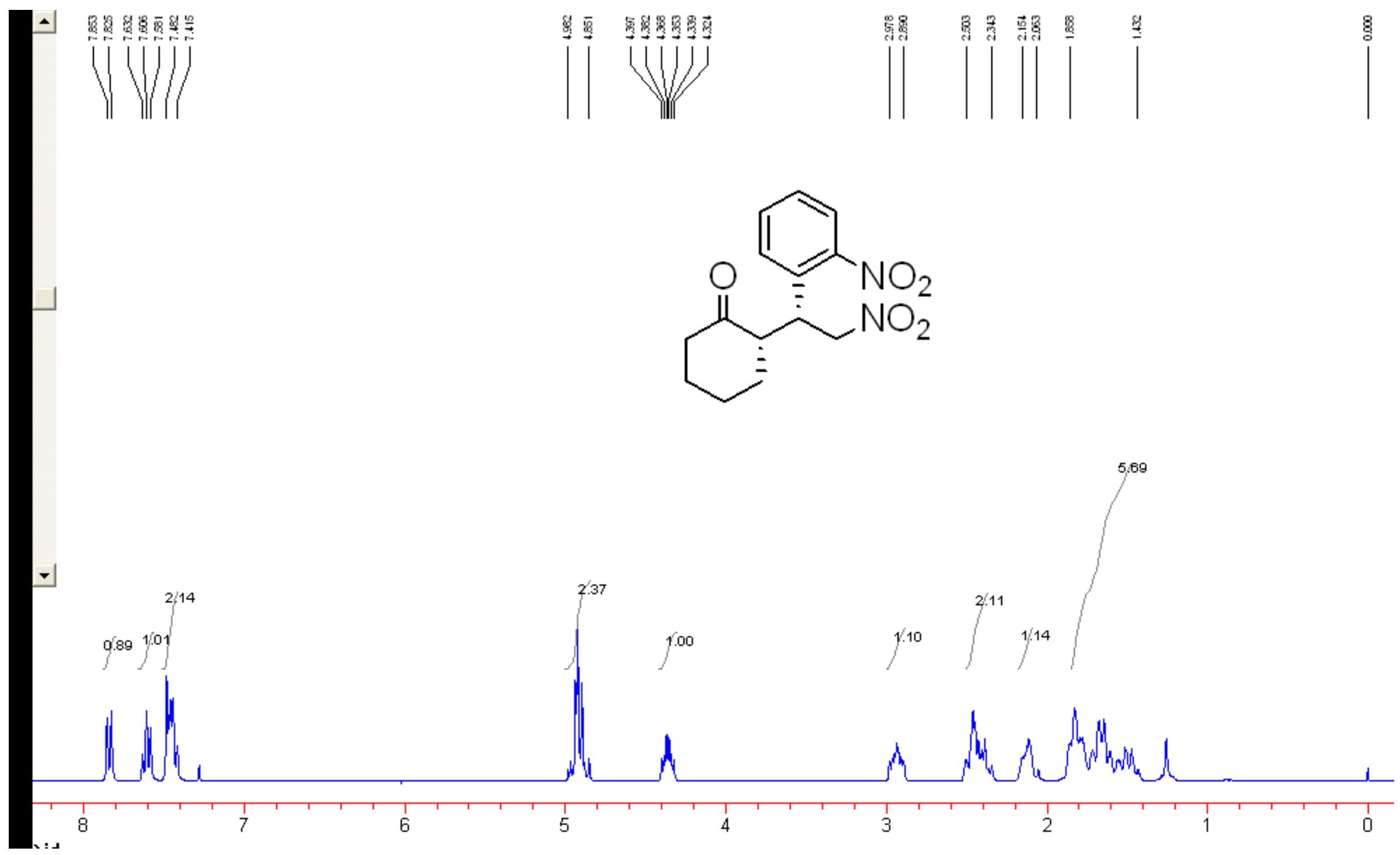

S15 
ce1-5-64b

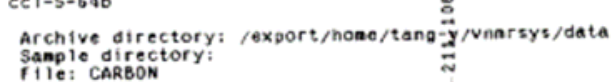

Pulse Sequence: s2pul

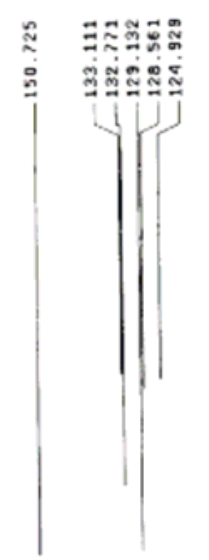

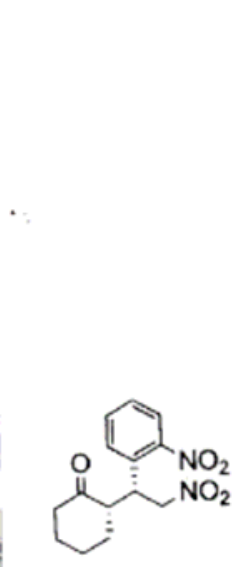

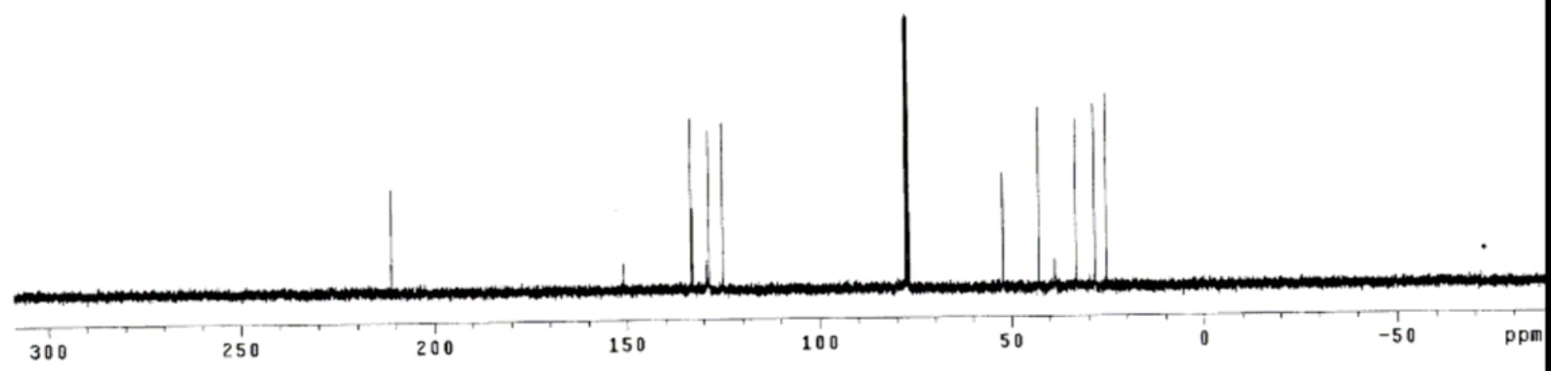



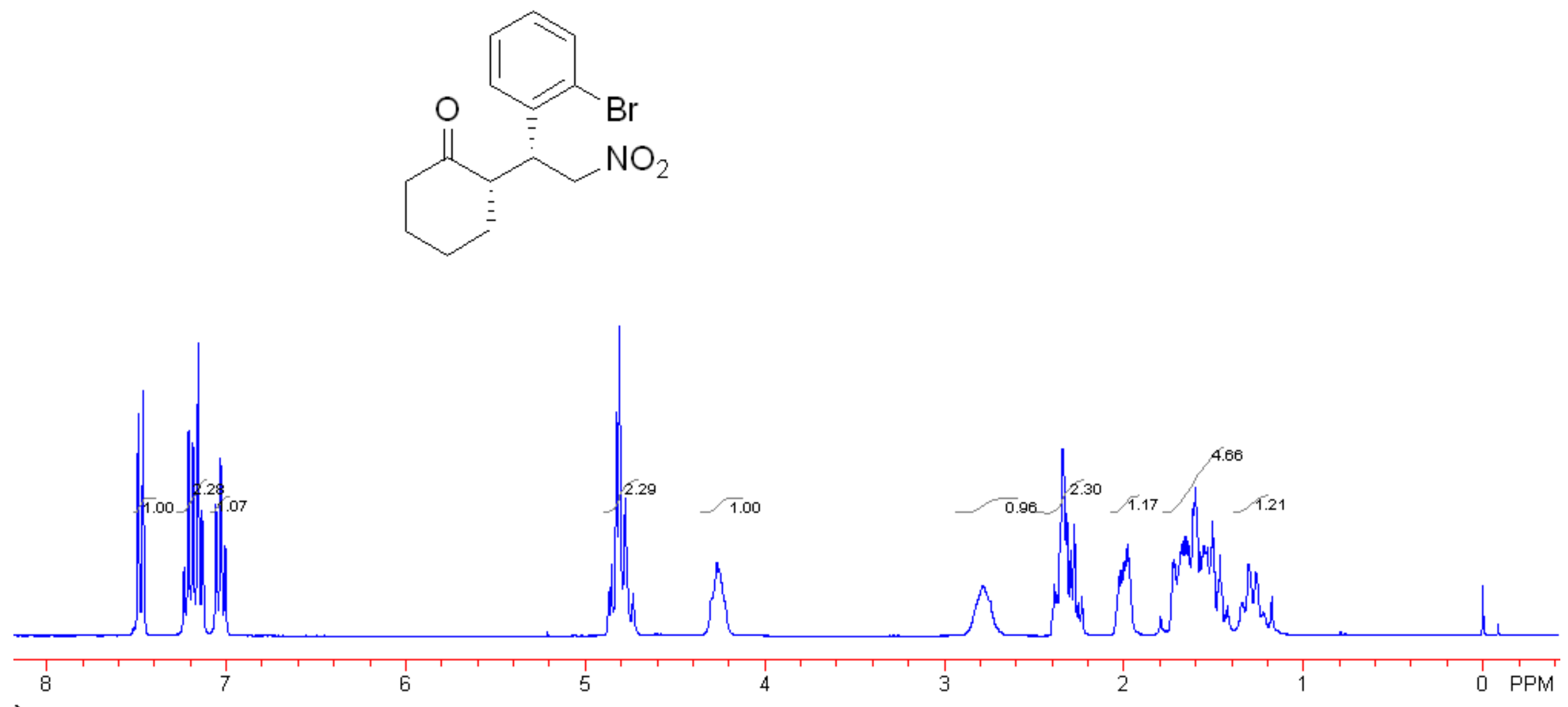


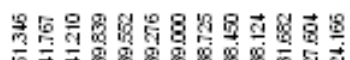

(
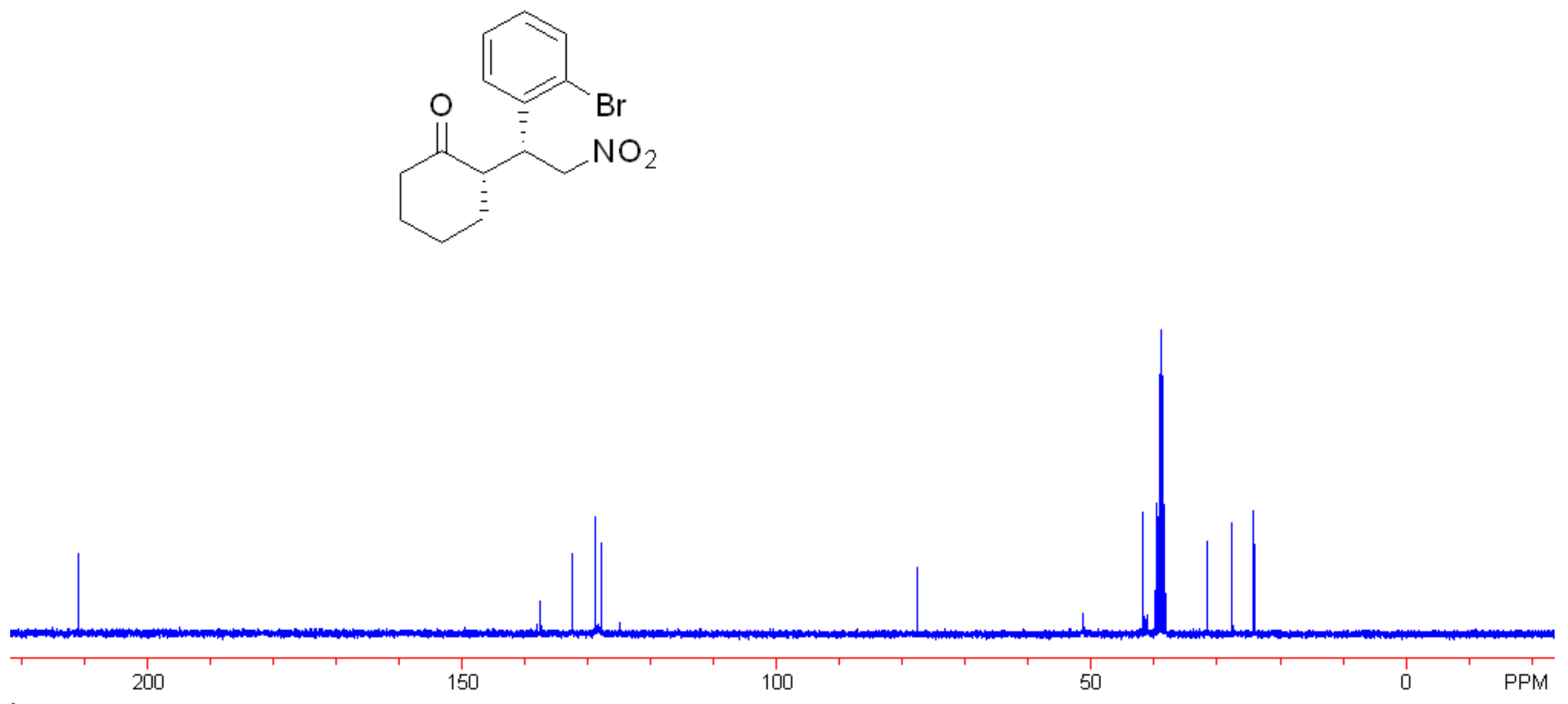

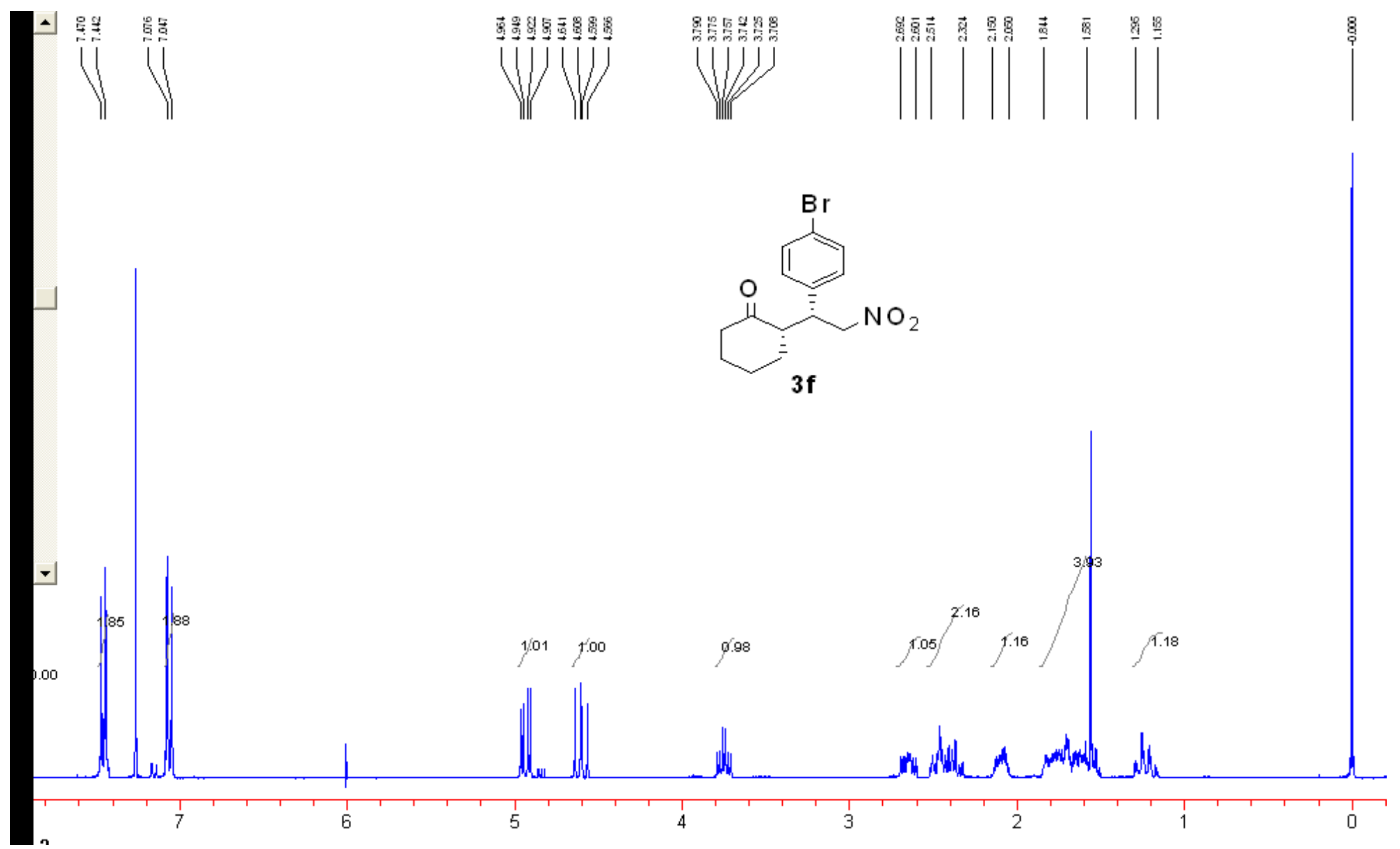

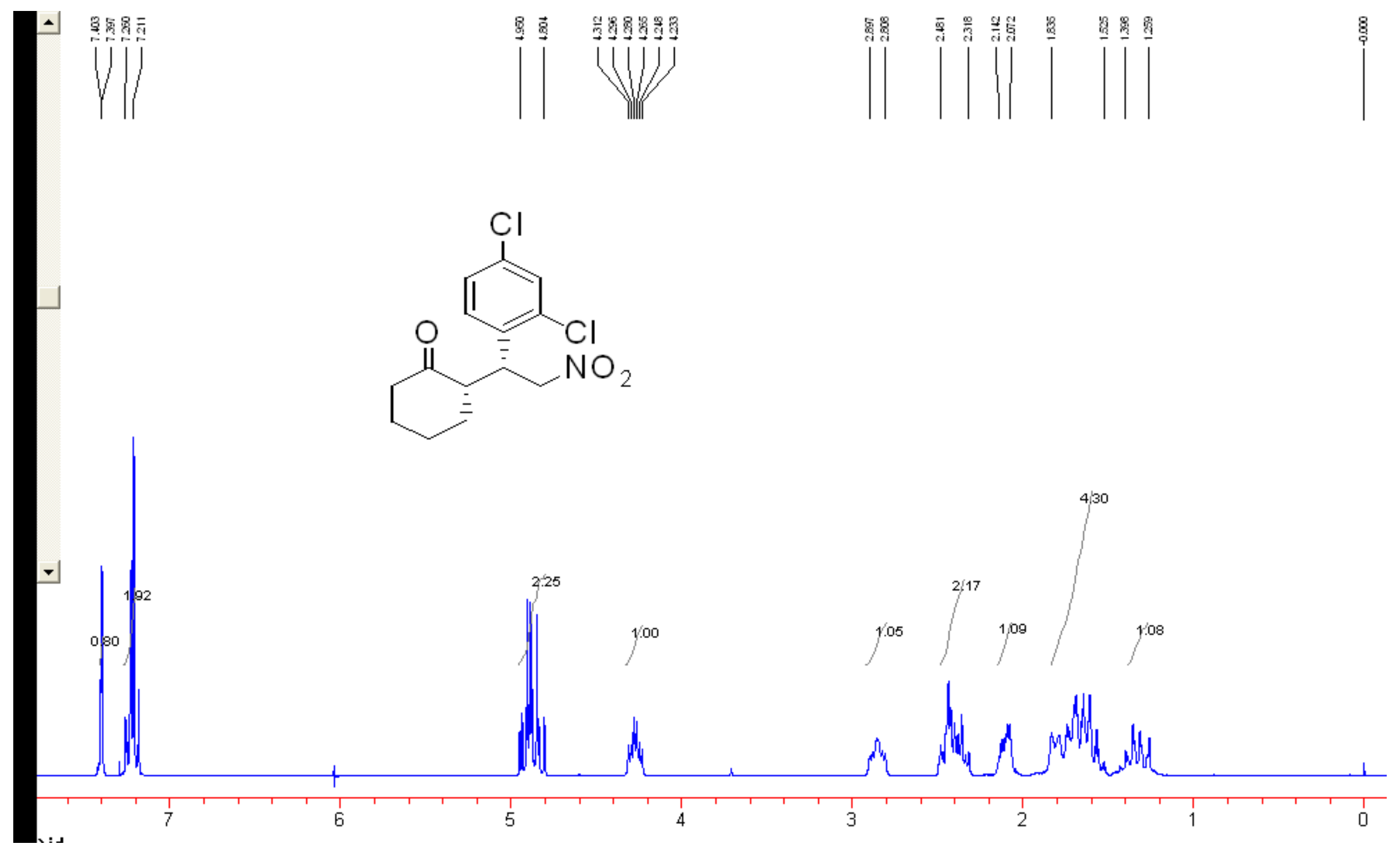

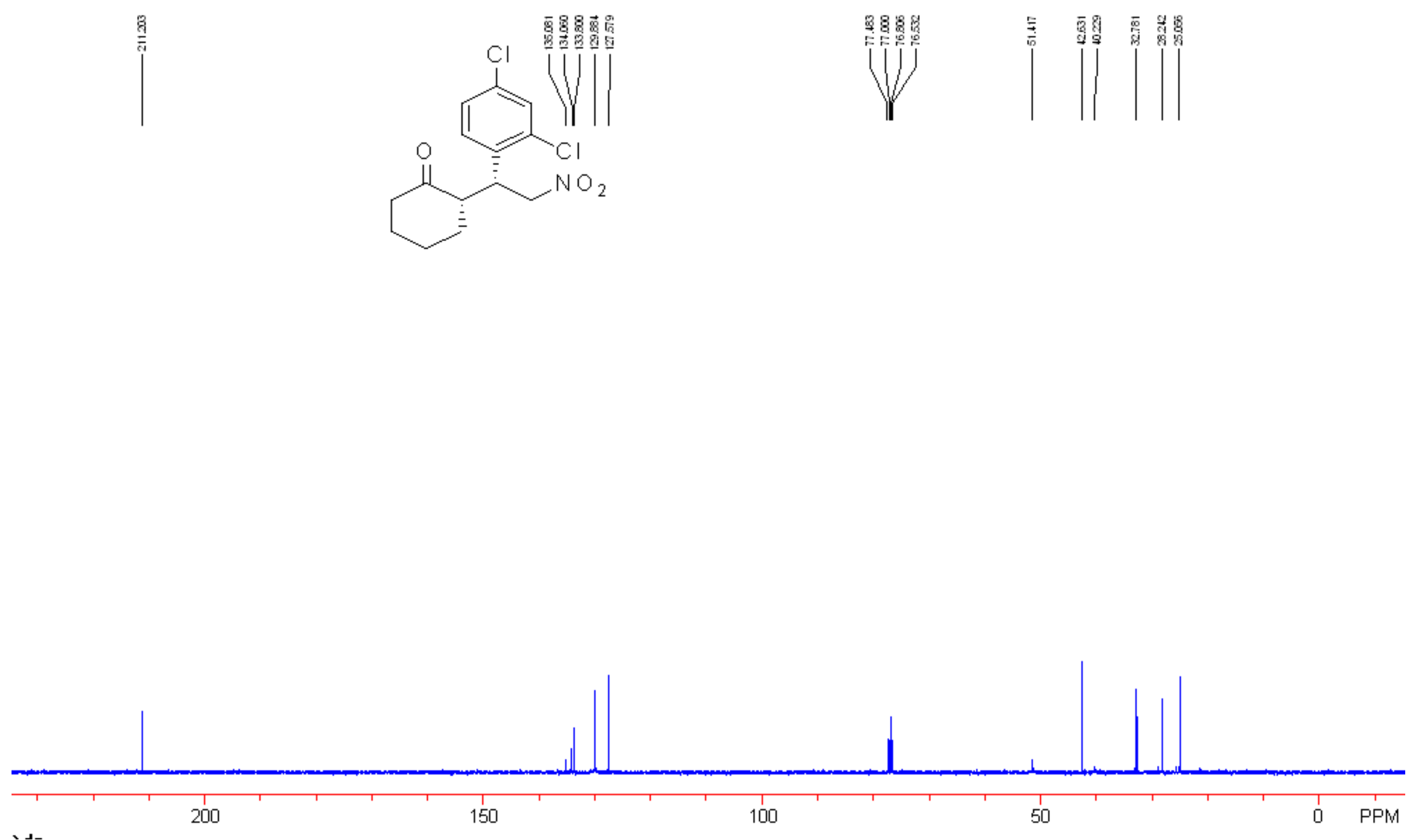


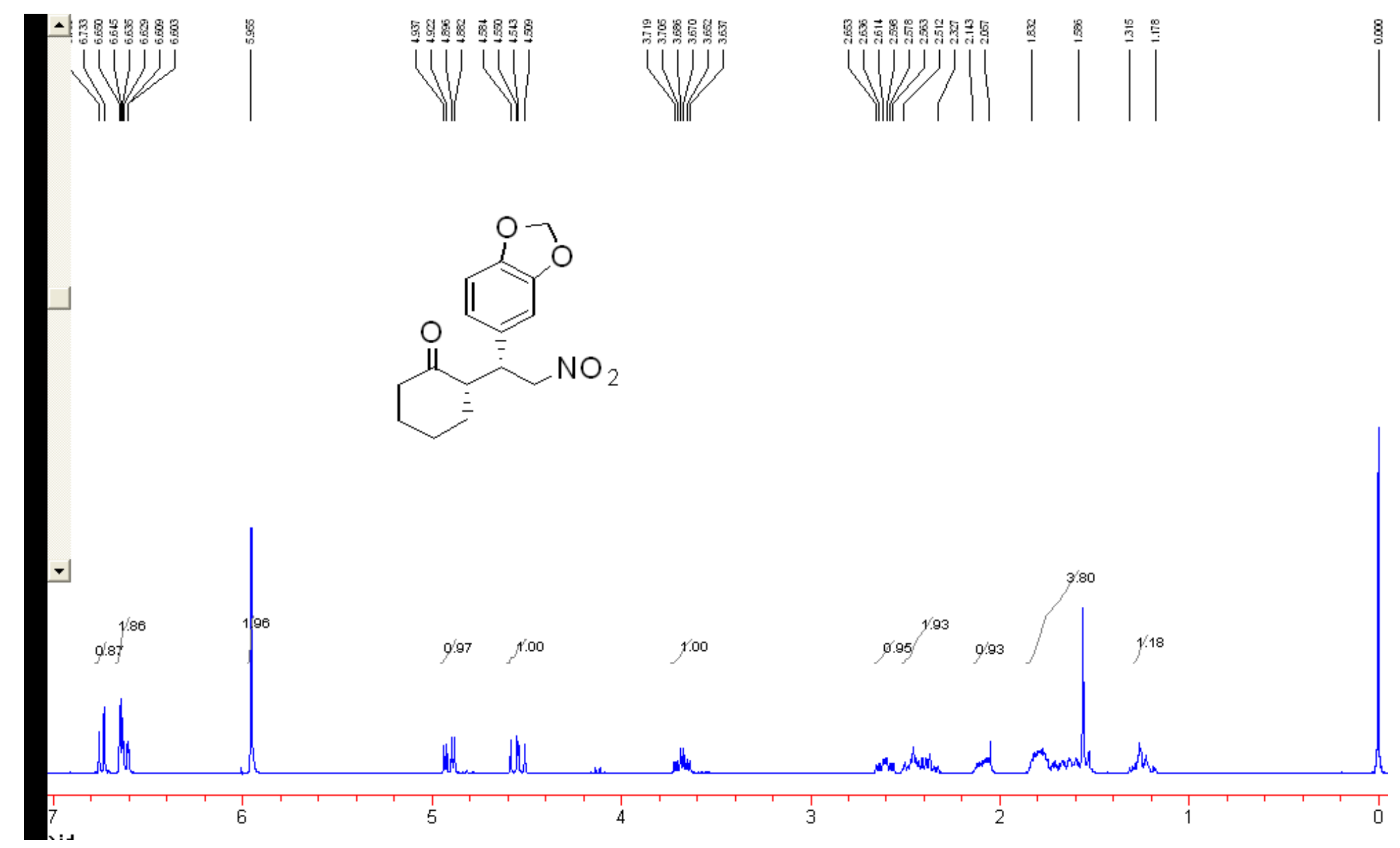



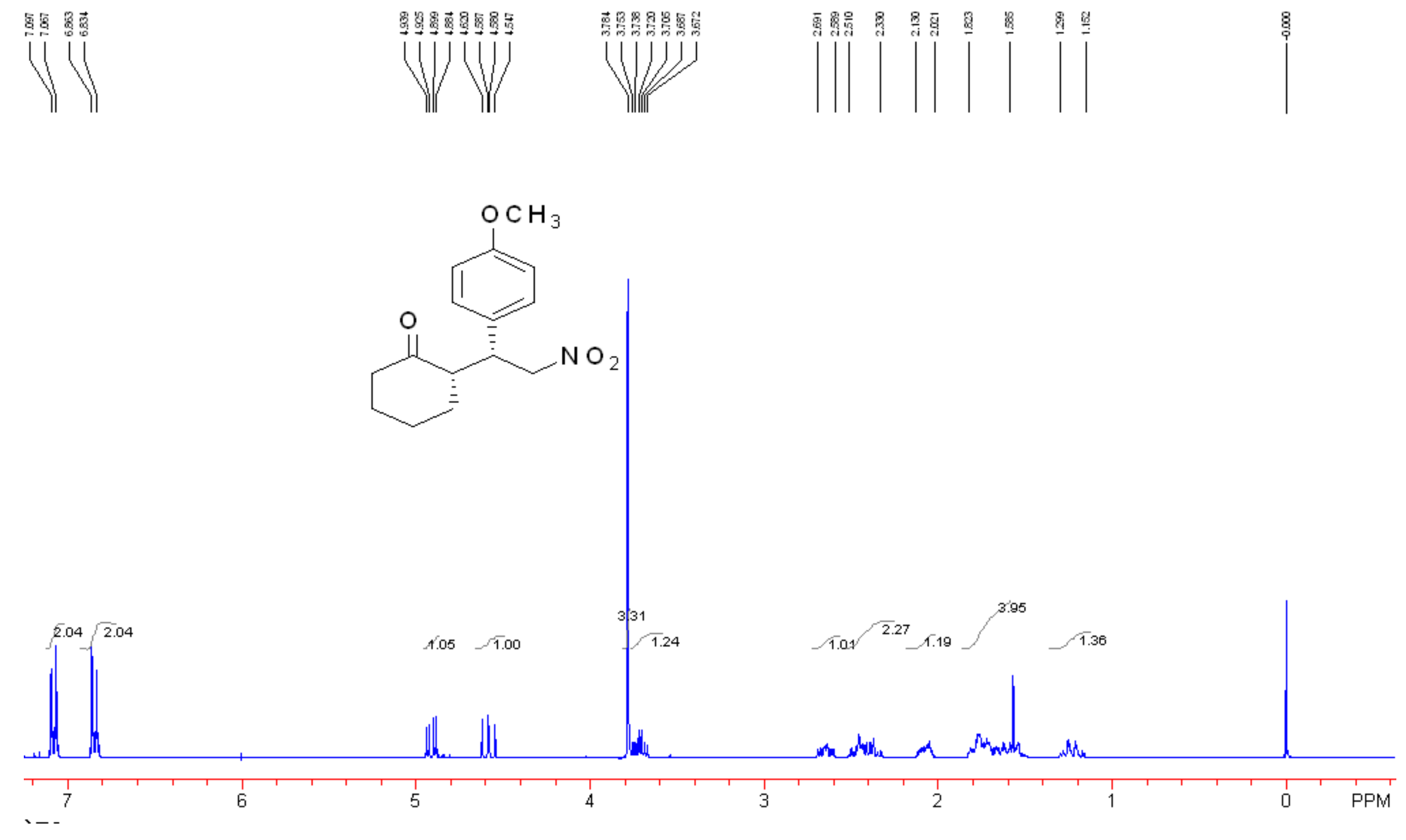


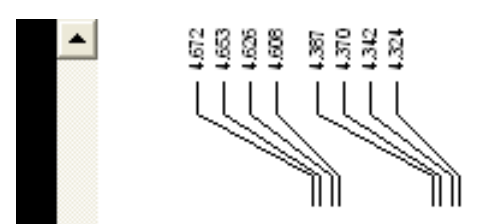

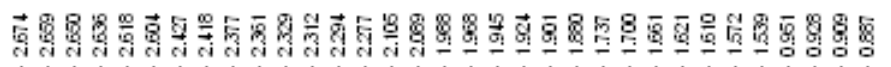

Lu(u) y
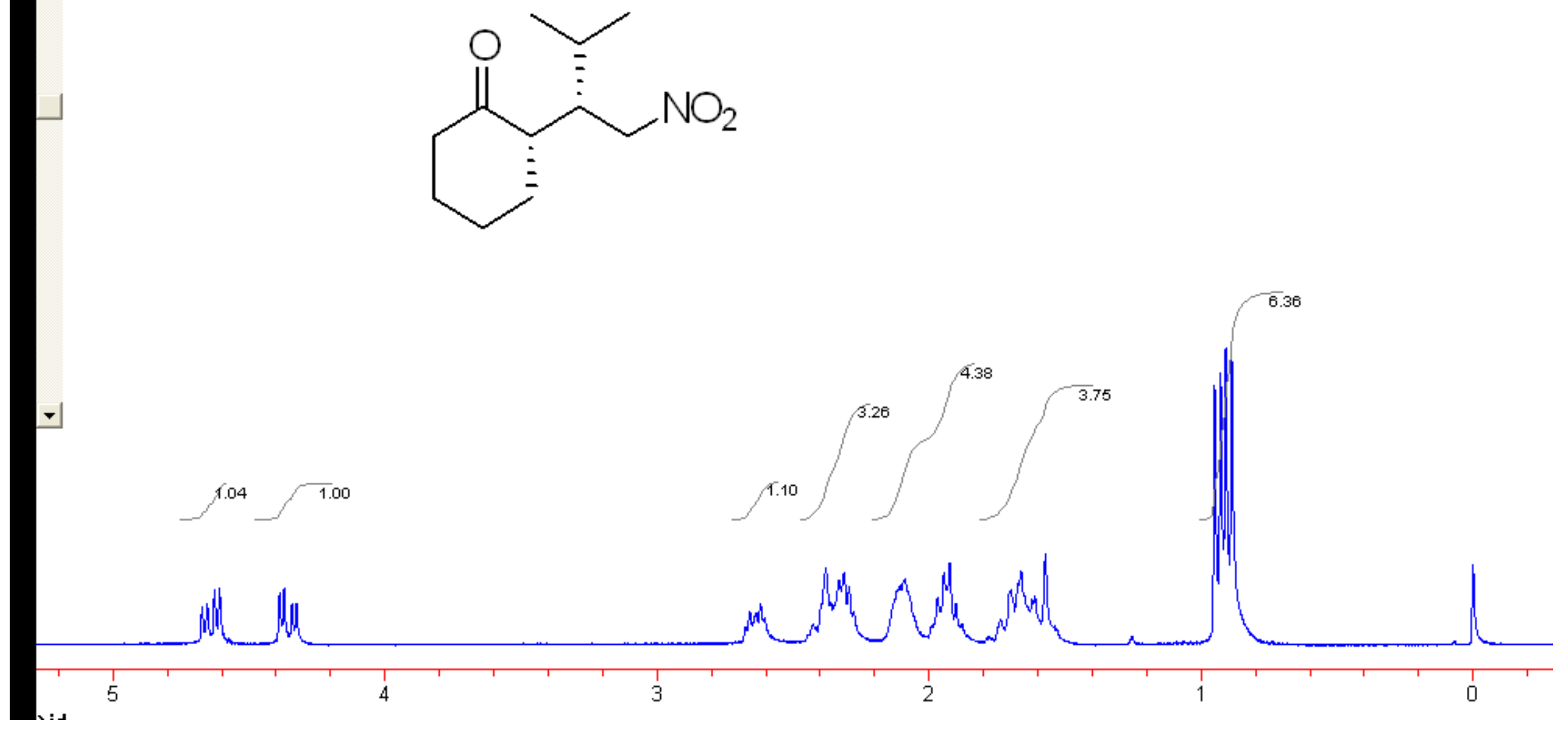

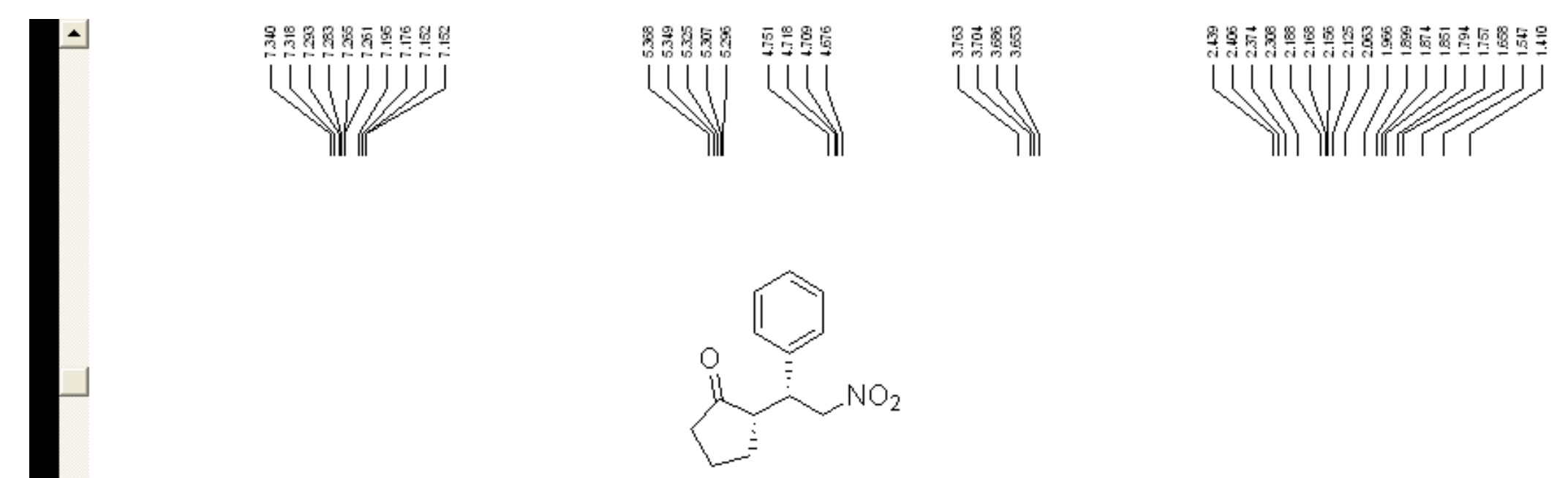

(S)-2-((R)-2-nitro-1-phenylethyl)cyclopentanone

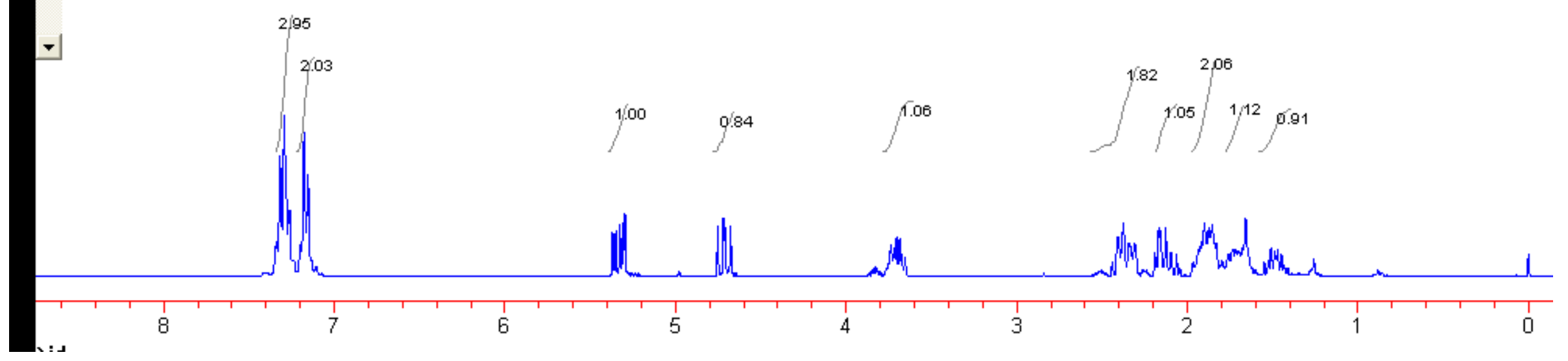



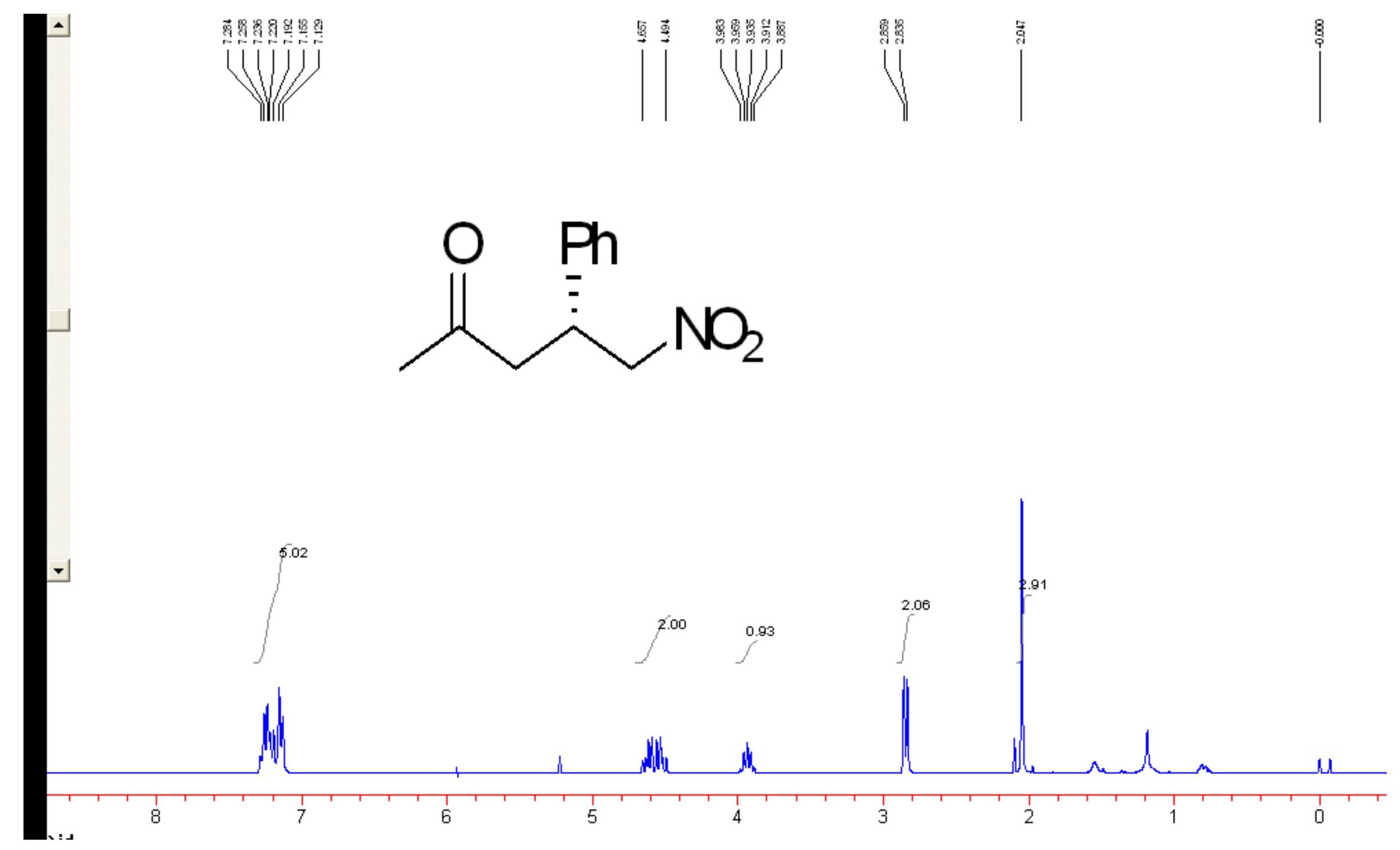

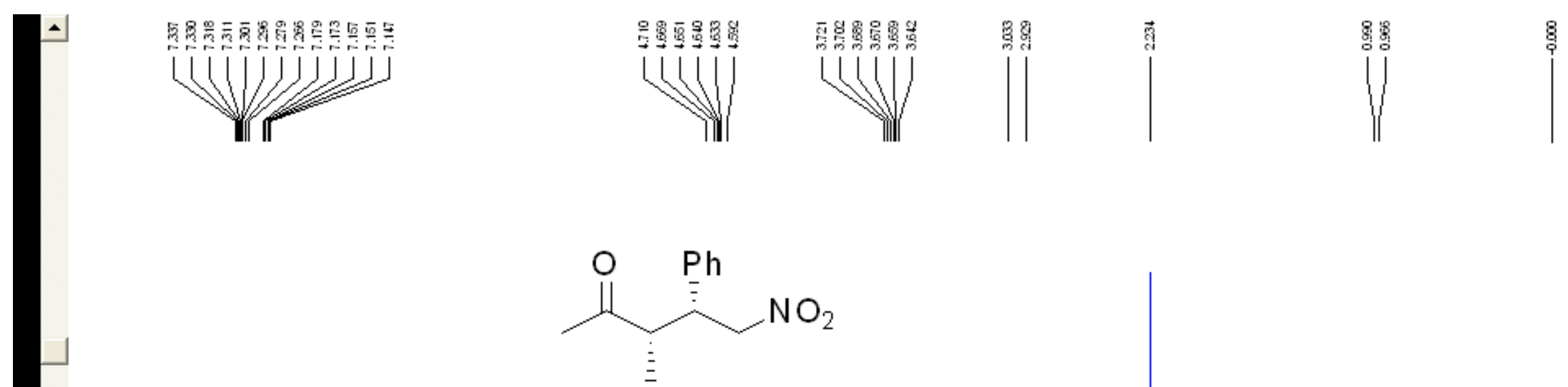

\section{Chemical Formula: $\mathrm{C}_{12} \mathrm{H}_{15} \mathrm{NO}_{3}$}

Molecular Weight: 221.3
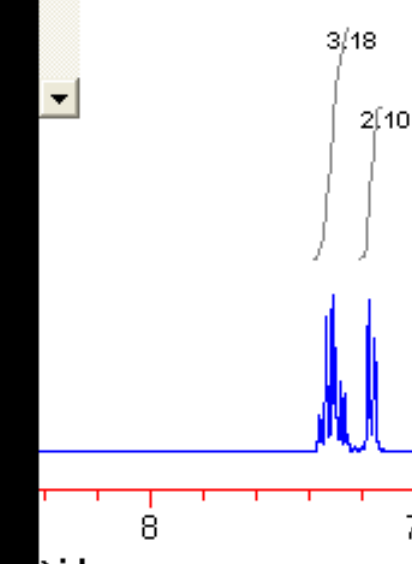

18 10 

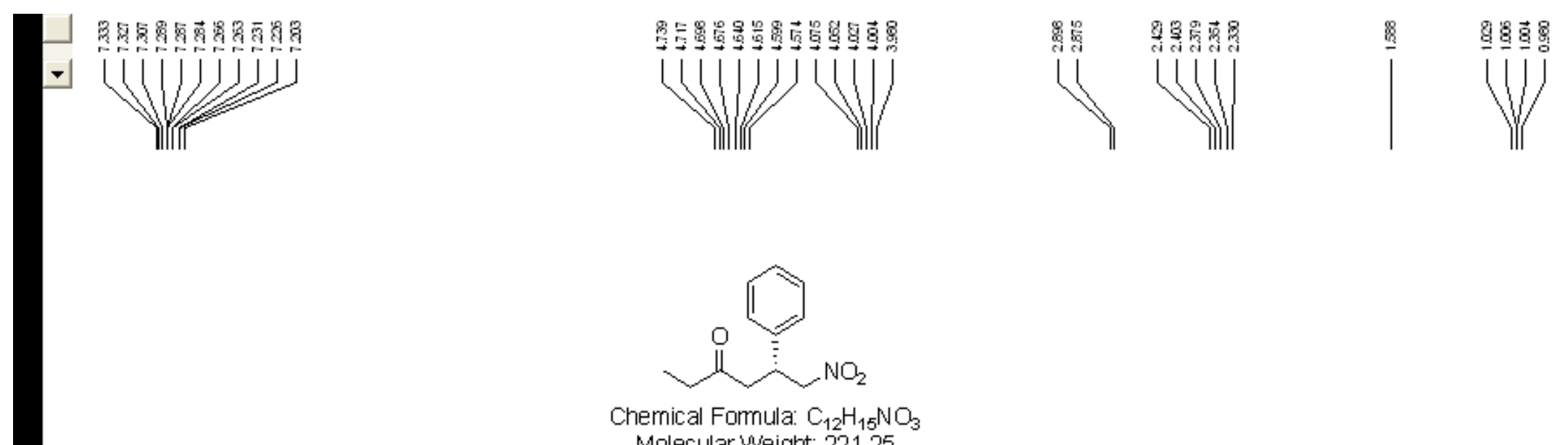

Molecular

Elemental Analysis: $\mathrm{C}, 65.14 ; \mathrm{H}, 6.83 ; \mathrm{N}, 6.33 ; 0,21.69$

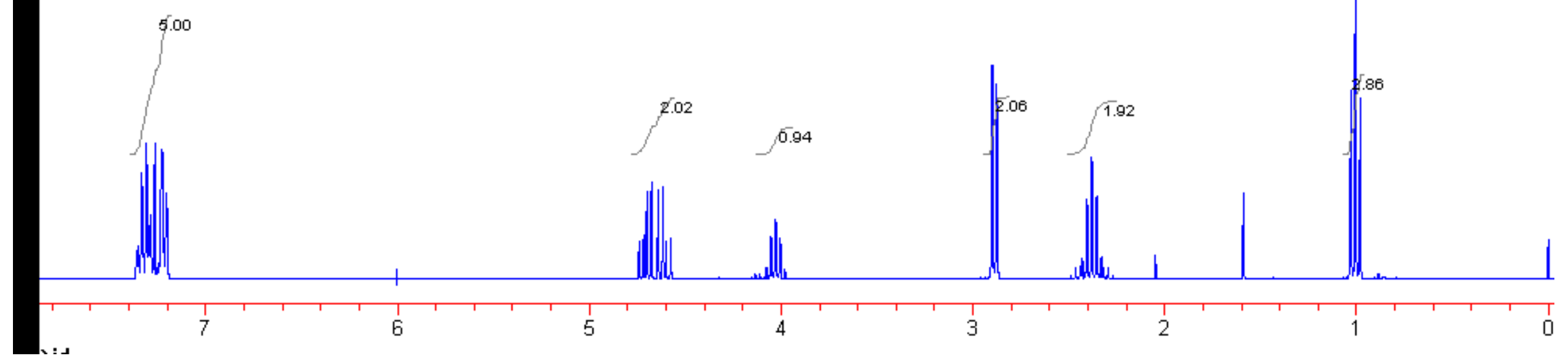




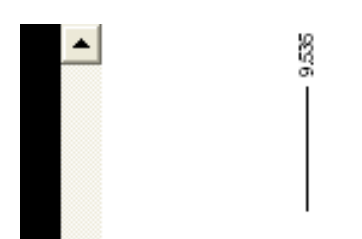

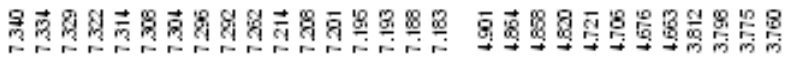

UUUWJJ)
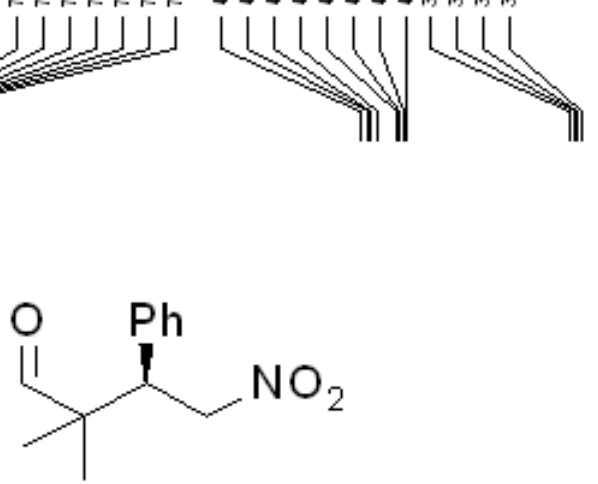

Chemical Formula: $\mathrm{C}_{12} \mathrm{H}_{15} \mathrm{NO}_{3}$

Molecular Weight: 221.3
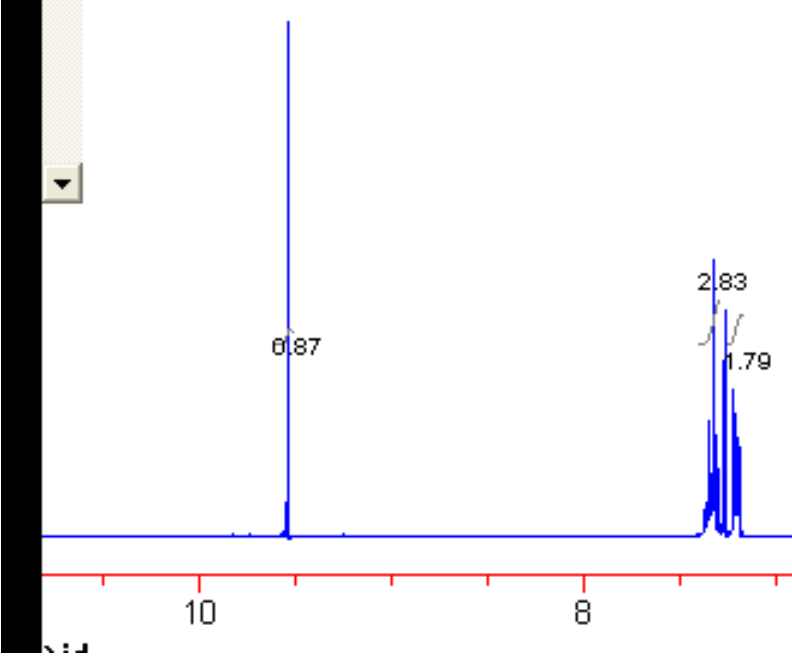

1.00

691

8

6 

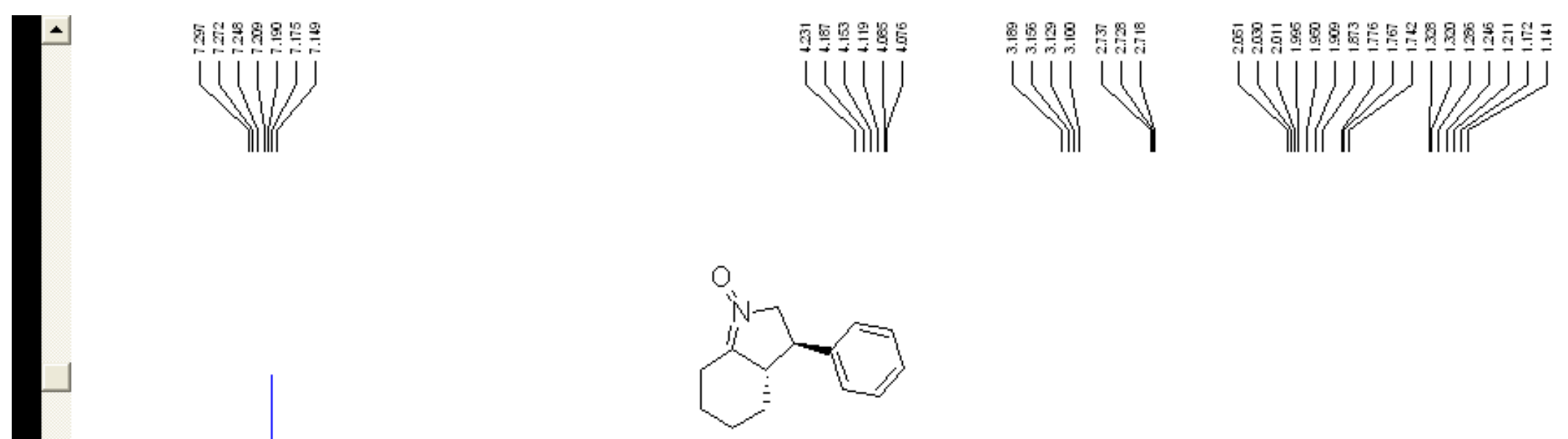

Chemical Formula: $\mathrm{C}_{14} \mathrm{H}_{17} \mathrm{NO}$

Molecular Weight: 215.29

Elemental Analysis: $C_{1} 78.10 ; H, 7.96 ; N, 6.51 ; 0,7.43$ 
$\Delta$

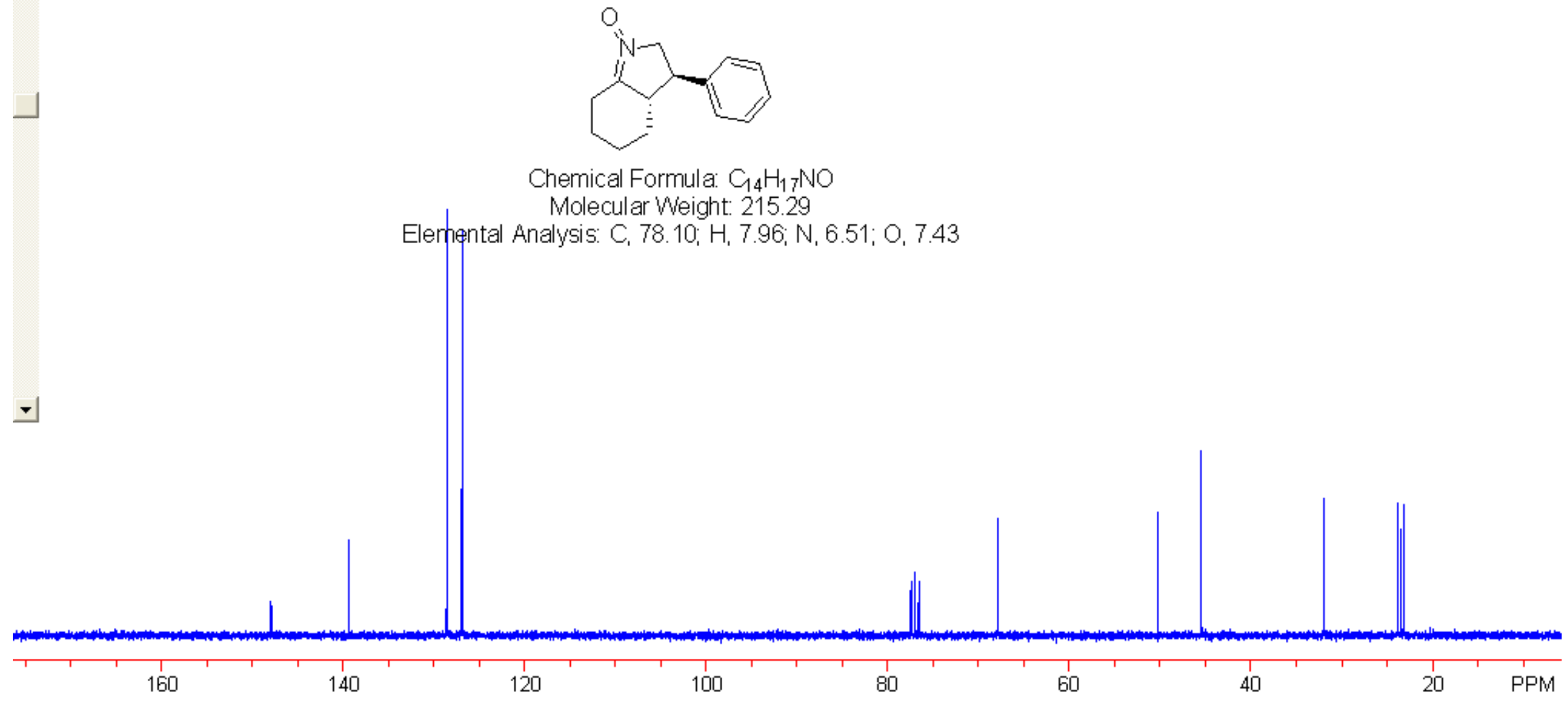



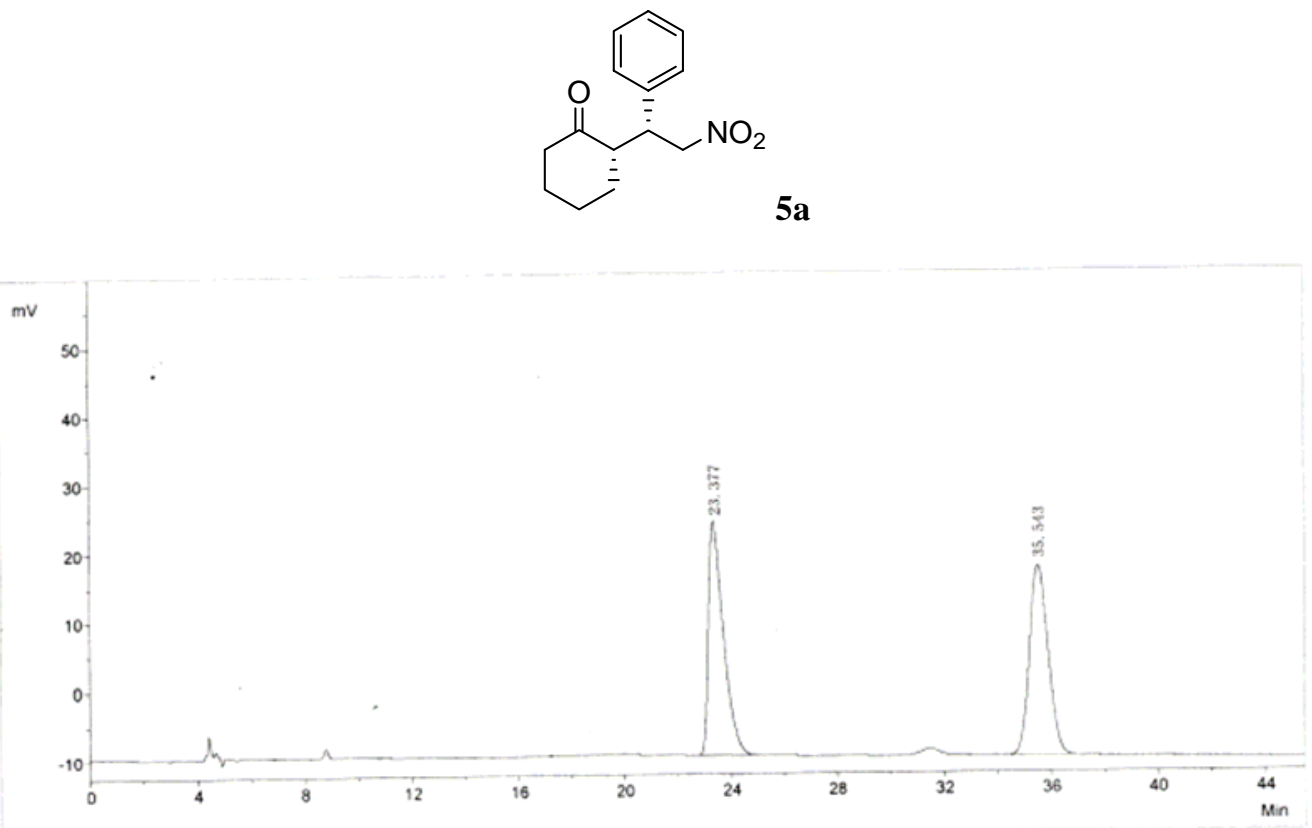

\begin{tabular}{rrrrr}
\multicolumn{1}{c}{ No. } & R. Time & PeakHeight & PeakArea & \multicolumn{1}{c}{ PerCent } \\
\hline 1 & 23.377 & 33661.7 & 1321860.2 & 49.9768 \\
2 & 35.543 & 27397.3 & 1323087.5 & 50.0232 \\
\hline Total & 61059.0 & 2644947.7 & 100.0000
\end{tabular}

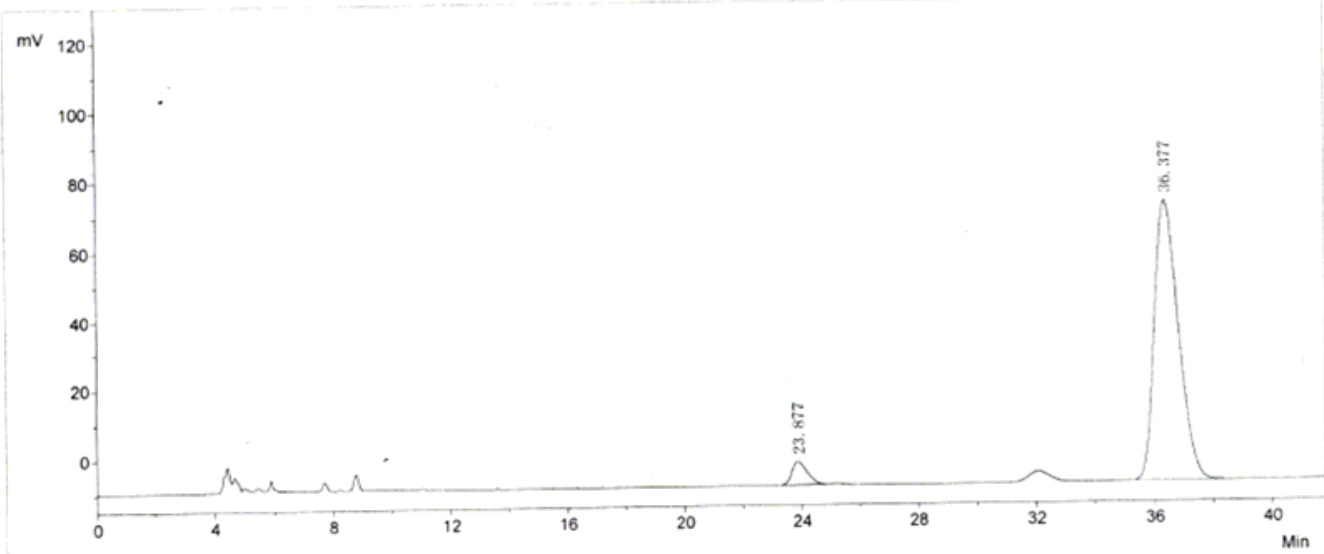

\begin{tabular}{rrrrr}
\multicolumn{1}{r}{ No. } & R. Time & PeakHeight & PeakArea & PerCent \\
\hline 1 & 23.877 & 658.5 .1 & 238170.5 & 4.9749 \\
2 & 36.377 & 80529.7 & 4549228.7 & 95.0251 \\
\hline Total & 87114.8 & 4787399.2 & 100.0000
\end{tabular}




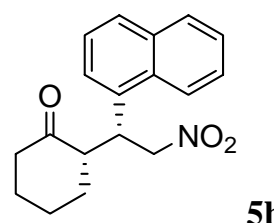

$5 b$

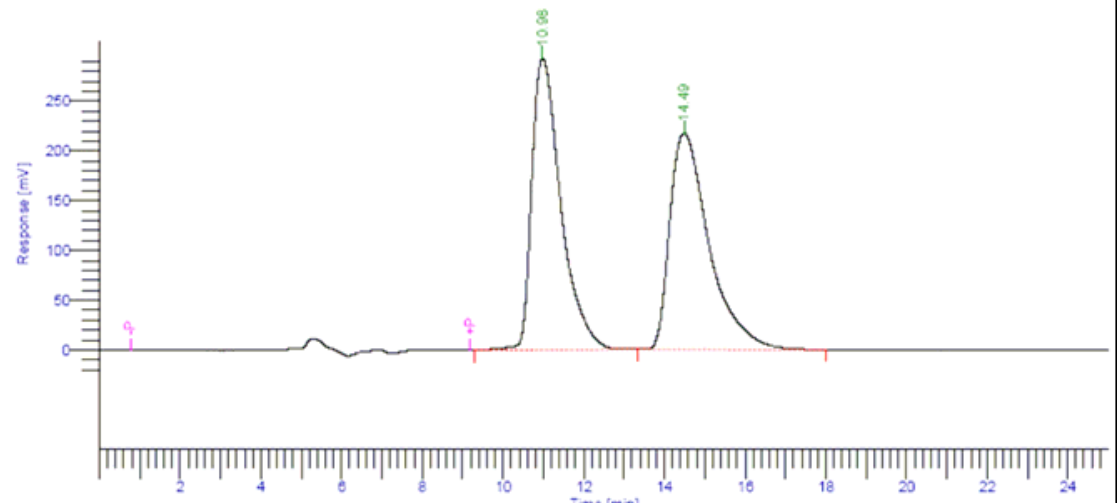

\section{$\mathrm{ccl}$}

ccl-5-80a-race

Peak Time Area Height Area Norm. Area

${ }_{1}^{\#} \frac{[\mathrm{min}]}{10.98} \frac{\left[\mathrm{uV}{ }^{*} \mathrm{sec}\right]}{1.4704 \mathrm{e}+07} \frac{[\mathrm{uV}]}{2.933 \mathrm{e}+05} \frac{[\%]}{49.93} \frac{[\%]}{49.93}$

$214.49 \underline{1.4745 e+07} \underline{2.177 \mathrm{e}+05} \underline{50.07}$ $\overline{2.9449 e+07} \overline{5.111 e+05} \overline{100.00} \quad 100.00$

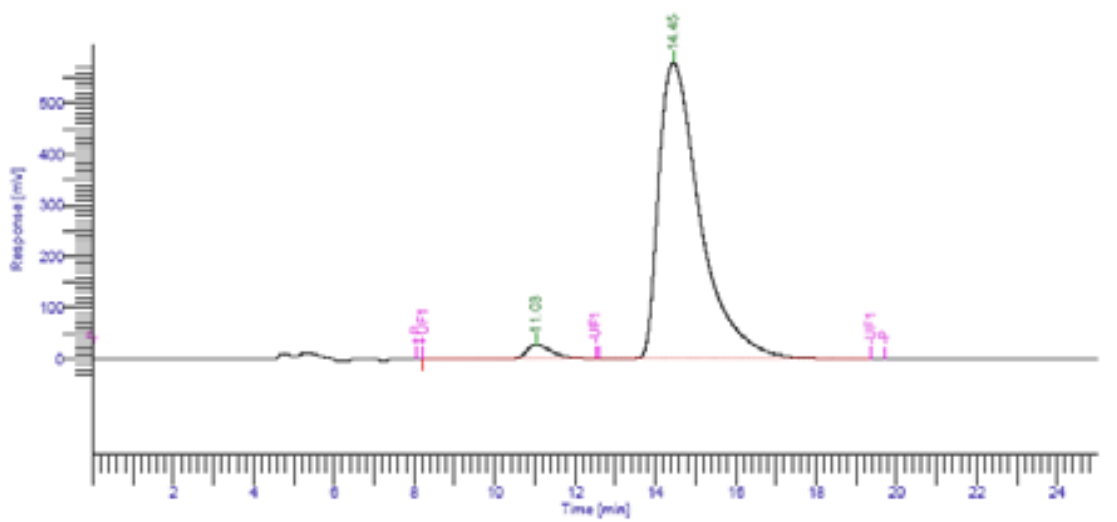

$\mathrm{ccl}$

cel-5-80a-biao

Peak Time Area Height Area Norm, Area

\begin{tabular}{|c|c|c|c|c|c|}
\hline II & [min] & [uv'sec] & [uV] & [\%] & {$[\%]$} \\
\hline \multirow{3}{*}{2} & 11.03 & $1.1321 \mathrm{e}+06$ & $2.658 \mathrm{e}+04$ & 2.67 & 2.67 \\
\hline & 14.45 & $4.1249 e+07$ & $5.770 \mathrm{e}+05$ & 97.33 & 97.33 \\
\hline & & 4.2381e+07 & $6.036 e+05$ & 100.00 & $100 \mathrm{c}$ \\
\hline
\end{tabular}



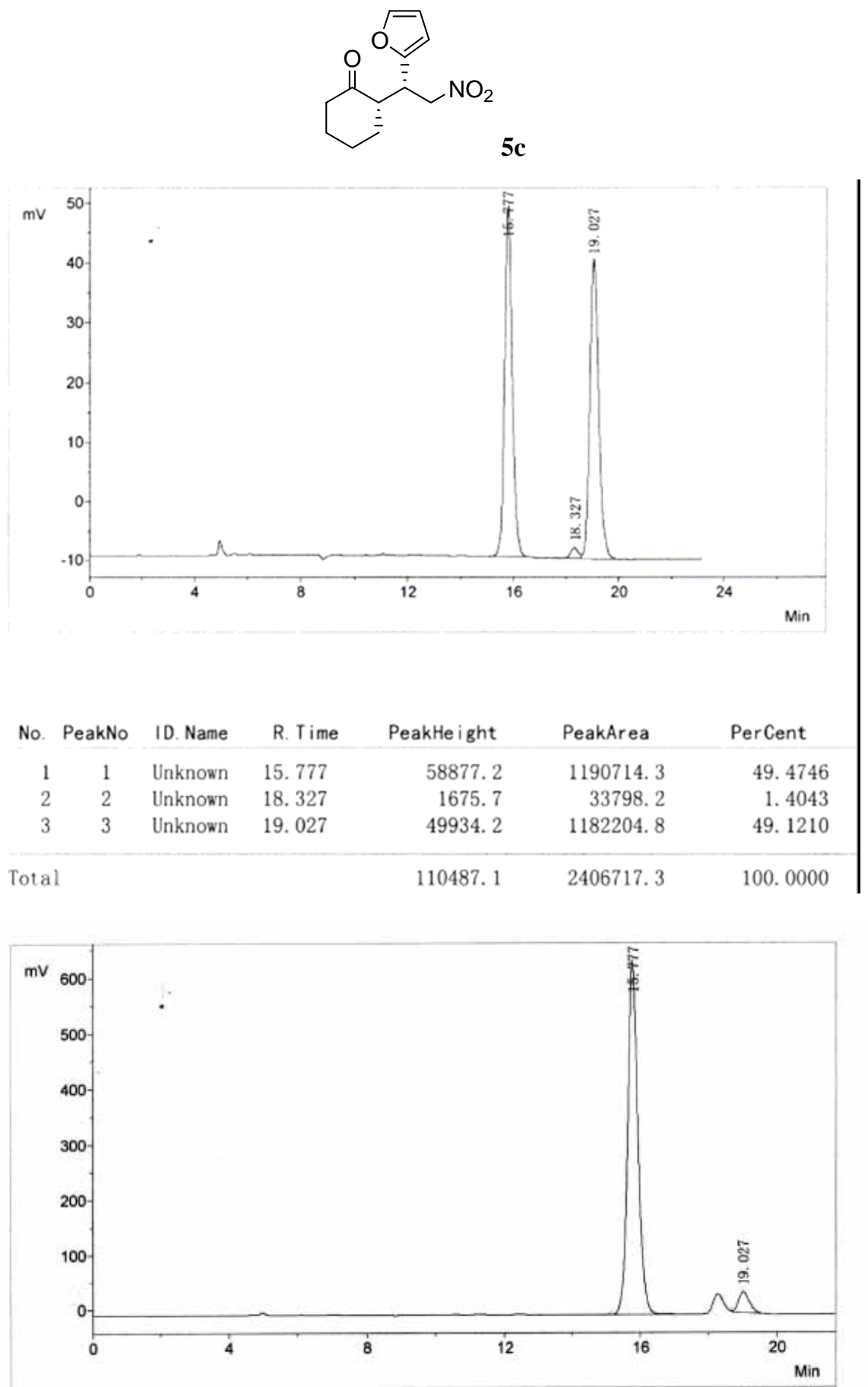

\begin{tabular}{rrrrrrr} 
No. PeakNo & ID. Name & R. Time & PeakHeight & PeakArea & PerCent \\
\hline 1 & 1 & Unknown & 15.777 & 633515.0 & 13270053.8 & 94.6858 \\
2 & 2 & Unknown & 19.027 & 35710.4 & 744778.0 & 5.3142 \\
Total & & & 669225.4 & 14014831.8 & 100.0000
\end{tabular}



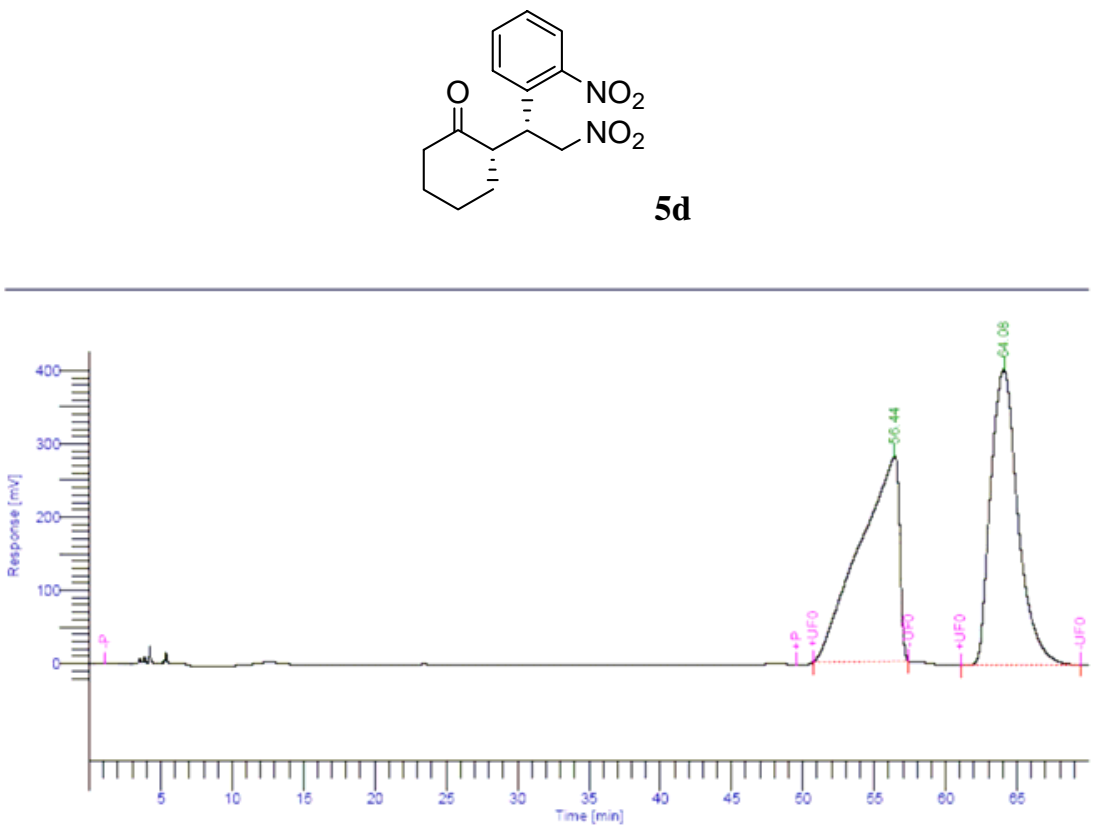

$\mathrm{ccl}$

ccl-5-79b-race

\begin{tabular}{|c|c|c|c|c|c|}
\hline $\begin{array}{c}\text { Peak } \\
\#\end{array}$ & $\begin{array}{l}\text { Time } \\
\text { [min] }\end{array}$ & $\begin{array}{c}\text { Area } \\
{\left[\mathrm{uV}^{*} \mathrm{sec}\right]}\end{array}$ & $\begin{array}{c}\text { Height } \\
\text { [uV] }\end{array}$ & $\begin{array}{c}\text { Area } \\
{[\%]}\end{array}$ & $\begin{array}{c}\text { Norm. Area } \\
{[\%]}\end{array}$ \\
\hline & $\begin{array}{l}56.44 \\
64.08\end{array}$ & $\begin{array}{l}5.3760 e+07 \\
5.3934 e+07\end{array}$ & $\begin{array}{l}2.800 \mathrm{e}+05 \\
4.032 \mathrm{e}+05\end{array}$ & $\begin{array}{l}49.92 \\
50.08\end{array}$ & $\begin{array}{l}49.92 \\
50.08\end{array}$ \\
\hline & & & & & \\
\hline
\end{tabular}

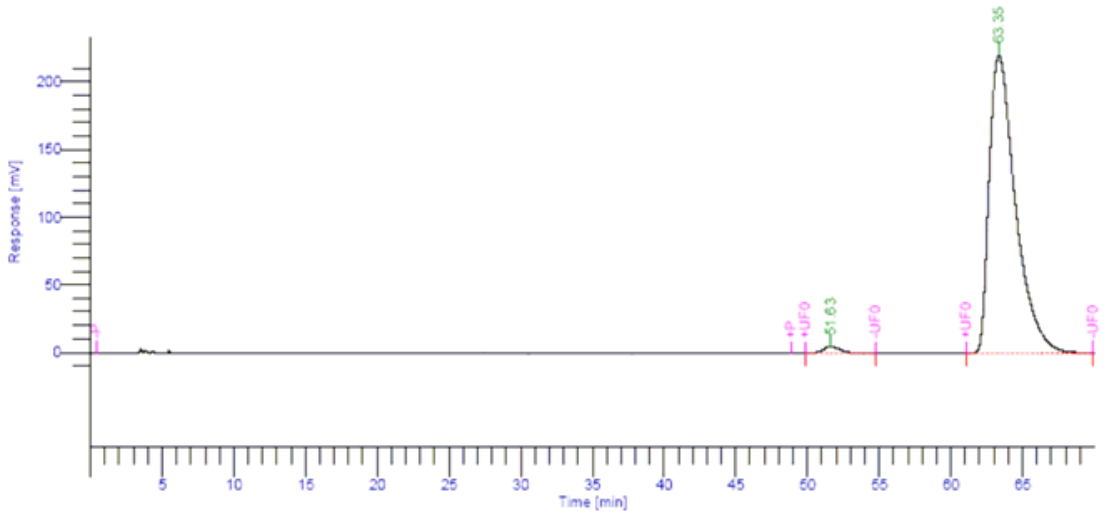

$\mathrm{ccl}$

ccl-5-79b-biao

Peak Time Area Height Area Norm. Area

\begin{tabular}{|c|c|c|c|c|c|}
\hline $\begin{array}{l}\text { eak } \\
\#\end{array}$ & $\begin{array}{l}\text { Time } \\
\text { [min] }\end{array}$ & $\begin{array}{c}\text { Area } \\
\text { [uv*sec] }\end{array}$ & $\begin{array}{l}\text { Height } \\
\text { [uV] }\end{array}$ & $\begin{array}{l}\text { Area } \\
{[\%]}\end{array}$ & $\begin{array}{c}\text { Norm. Area } \\
{[\%]}\end{array}$ \\
\hline & \multirow{3}{*}{$\begin{array}{ll}1 & 51.63 \\
2 & 63.35\end{array}$} & 442 & & & 1.55 \\
\hline & & $2.8145 \mathrm{e}+07$ & $2.206 \mathrm{e}$ & 98.45 & 98.4 \\
\hline & & $2.8587 e+07$ & $2.256 \mathrm{e}+05$ & 100.00 & 100.00 \\
\hline
\end{tabular}




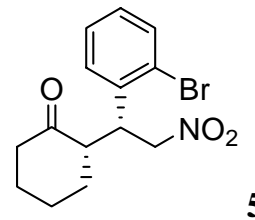

$5 e$

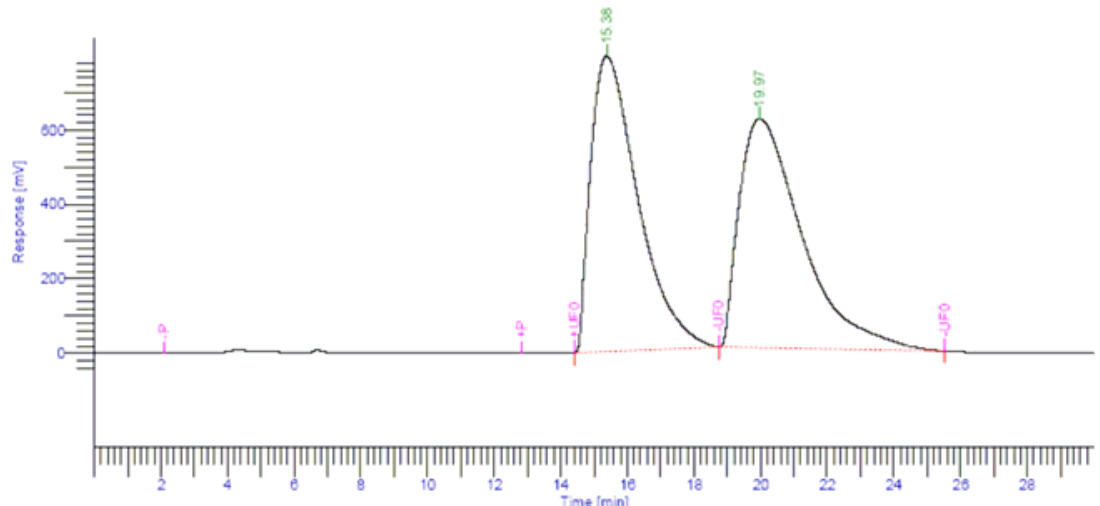

ccl

ccl-5-83a-race

\begin{tabular}{|c|c|c|c|c|c|}
\hline $\begin{array}{c}\text { Peak } \\
\#\end{array}$ & $\begin{array}{l}\text { Time } \\
\text { [min] }\end{array}$ & $\begin{array}{c}\text { Area } \\
{\left[\mathrm{uV}^{2} \mathrm{sec}\right]}\end{array}$ & $\begin{array}{l}\text { Height } \\
\text { [uV] }\end{array}$ & $\begin{array}{c}\text { Area } \\
{[\%]}\end{array}$ & $\begin{array}{c}\text { Norm. Area } \\
{[\%]}\end{array}$ \\
\hline 1 & 15.38 & $7.8169 \mathrm{e}+07$ & $7.943 e$ & 49.24 & 49.24 \\
\hline 2 & 19.97 & $8.0587 e+07$ & $6.157 \mathrm{e}+05$ & 50.76 & 50.76 \\
\hline
\end{tabular}

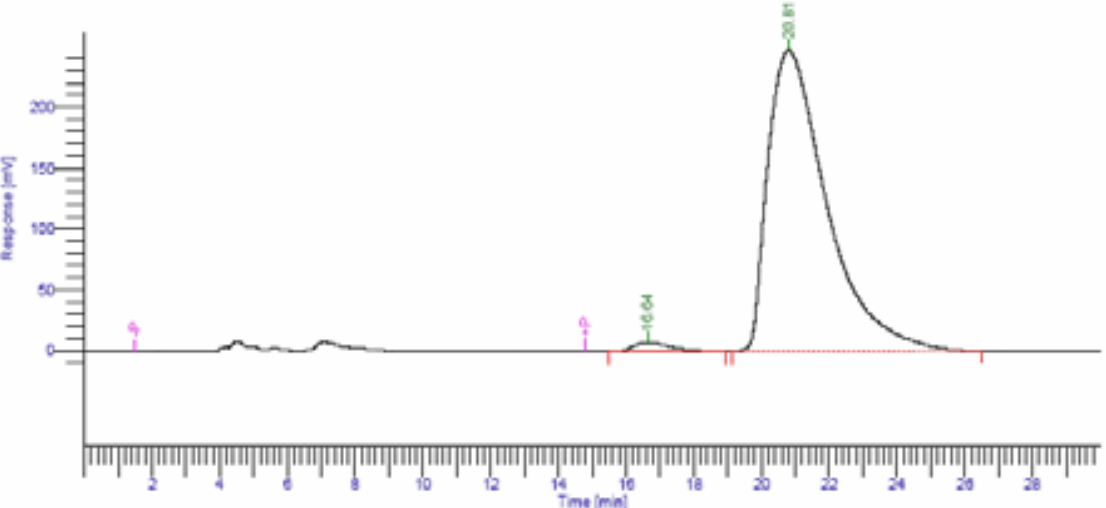

ccl

ccl-5-83a-race

\begin{tabular}{|c|c|c|c|c|c|}
\hline $\begin{array}{l}\text { eak } \\
*\end{array}$ & $\begin{array}{l}\text { Time } \\
\text { [min] }\end{array}$ & $\begin{array}{c}\text { Area } \\
{\left[u^{*} v^{*} \sec \right]}\end{array}$ & $\begin{array}{l}\text { Height } \\
\text { [uV] }\end{array}$ & $\begin{array}{l}\text { Area } \\
{[\%]}\end{array}$ & $\begin{array}{c}\text { Norm. Area } \\
{[\%]}\end{array}$ \\
\hline $\begin{array}{l}1 \\
2\end{array}$ & \multirow[t]{2}{*}{$\begin{array}{l}16.64 \\
20.81\end{array}$} & & & & $\begin{array}{r}1.78 \\
98.22\end{array}$ \\
\hline & & 0500 & $40 e+05$ & 0.00 & 100.00 \\
\hline
\end{tabular}


<smiles>O=C1CCCC[C@H]1C(C[N+](=O)[O-])c1ccc(Br)cc1</smiles>

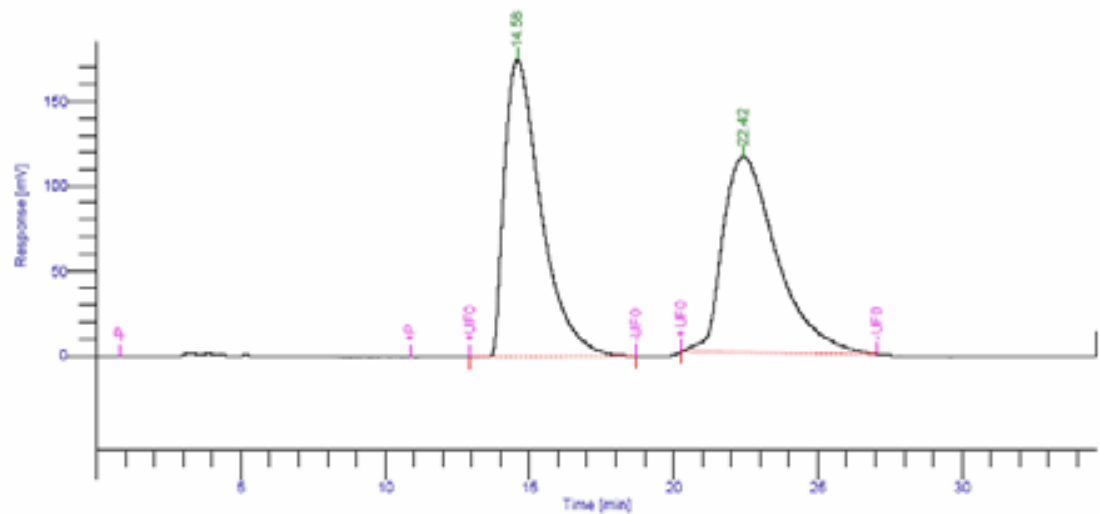

ccl

ccl-5-80b-race

Peak Time Area Height Area Norm. Area

\# [min] $\left[\mathrm{uV}^{*} \mathrm{sec}\right]$

$114.58 \frac{1.5361 \mathrm{e}+07}{1.750 \mathrm{e}+05} \overline{50.53} \frac{50.53}{4.53}$

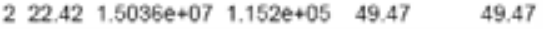

$\overline{3.0397 e+07} \overline{2.902 e+05} \overline{100.00} \overline{100.00}$

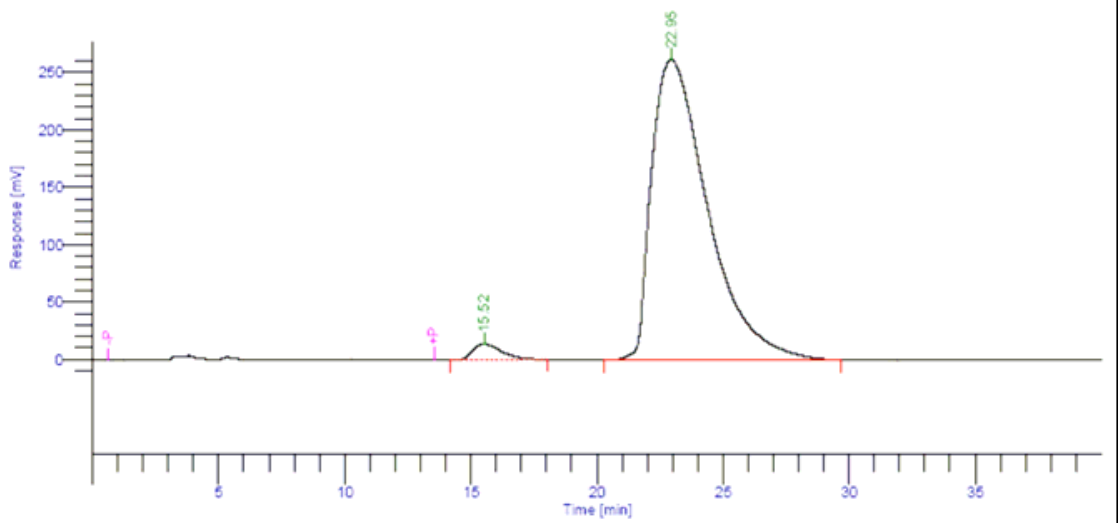

$\mathrm{ccl}$

ccl-5-80b-biao

Peak Time Area Height Area Norm. Area

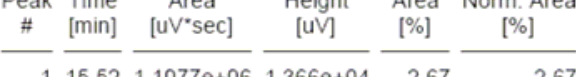

$\begin{array}{rrrrrr}1 & 15.52 & 1.1077 \mathrm{e}+06 & 1.366 \mathrm{e}+04 & 2.67 & 2.67 \\ 2 & 22.95 & 4.0407 \mathrm{e}+07 & 2.614 \mathrm{e}+05 & 97.33 & 97.33\end{array}$

$\overline{4.1515 e+07} \overline{2.751 e+05} \overline{100.00} \overline{100.00}$ 

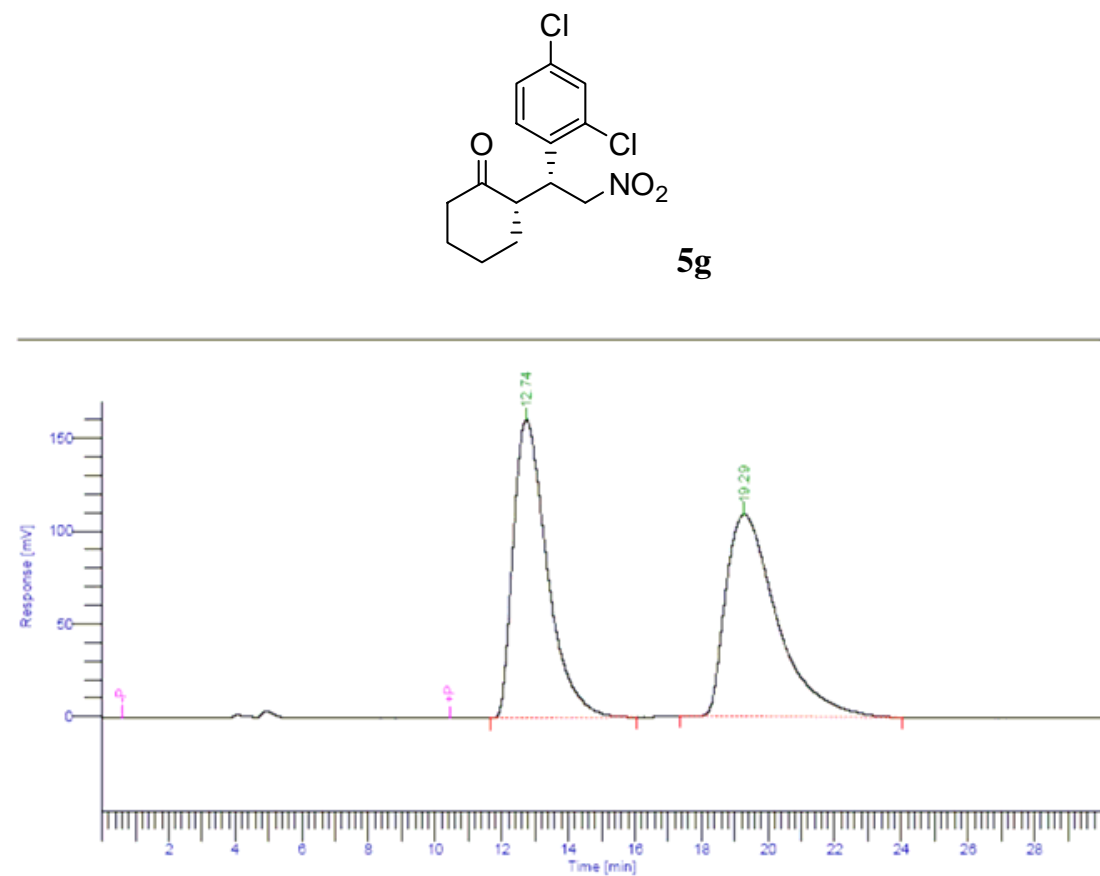

\section{ccl}

\begin{tabular}{|c|c|c|c|c|c|}
\hline \multicolumn{6}{|c|}{ ccl-5-84-race } \\
\hline $\begin{array}{c}\text { Peak } \\
\#\end{array}$ & $\begin{array}{l}\text { Time } \\
\text { [min] }\end{array}$ & $\begin{array}{c}\text { Area } \\
\text { [uv*sec] }\end{array}$ & $\begin{array}{l}\text { Height } \\
\text { [uV] }\end{array}$ & $\begin{array}{l}\text { Area } \\
{[\%]}\end{array}$ & $\begin{array}{c}\text { Norm. Area } \\
{[\%]}\end{array}$ \\
\hline \multirow{3}{*}{$\begin{array}{l}1 \\
2\end{array}$} & 12.74 & $1.1415 \mathrm{e}+07$ & $1.608 \mathrm{e}+05$ & 49.60 & 49.60 \\
\hline & 19.29 & $1.1601 \mathrm{e}+07$ & $1.088 \mathrm{e}+05$ & 50.40 & 50.40 \\
\hline & & $2.3017 \mathrm{e}+07$ & $2.696 e+05$ & 100.00 & 100.00 \\
\hline
\end{tabular}

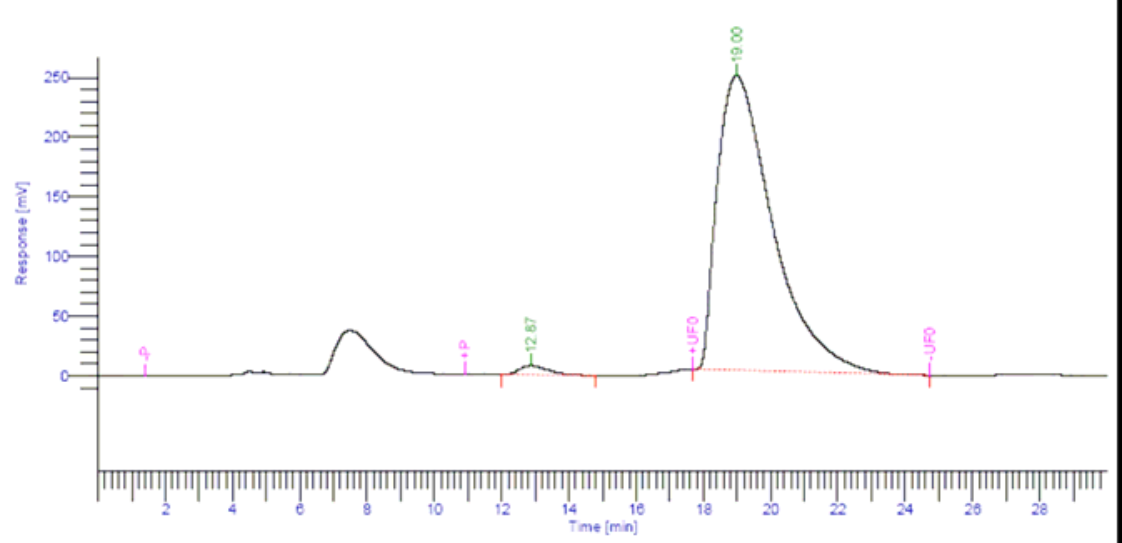

$\mathrm{ccl}$

\begin{tabular}{|c|c|c|c|c|c|}
\hline \multicolumn{6}{|c|}{ ccl-5-84-biao } \\
\hline $\begin{array}{c}\text { Peak } \\
\#\end{array}$ & $\begin{array}{l}\text { Time } \\
\text { [min] }\end{array}$ & $\begin{array}{c}\text { Area } \\
{\left[\mathrm{uV}^{*} \mathrm{sec}\right]}\end{array}$ & $\begin{array}{l}\text { Height } \\
{[\mathrm{uV}]}\end{array}$ & $\begin{array}{c}\text { Area } \\
{[\%]}\end{array}$ & $\begin{array}{c}\text { Norm. Area } \\
{[\%]}\end{array}$ \\
\hline \multirow{3}{*}{$\begin{array}{l}1 \\
2\end{array}$} & 12.87 & 467172.0400 & 7798.8437 & 1.63 & 1.63 \\
\hline & 19.00 & $2.8256 \mathrm{e}+07$ & $2.468 \mathrm{e}+05$ & 98.37 & 98.37 \\
\hline & & $2.8723 e+07$ & $2.546 \mathrm{e}+05$ & 100.00 & 100.00 \\
\hline
\end{tabular}



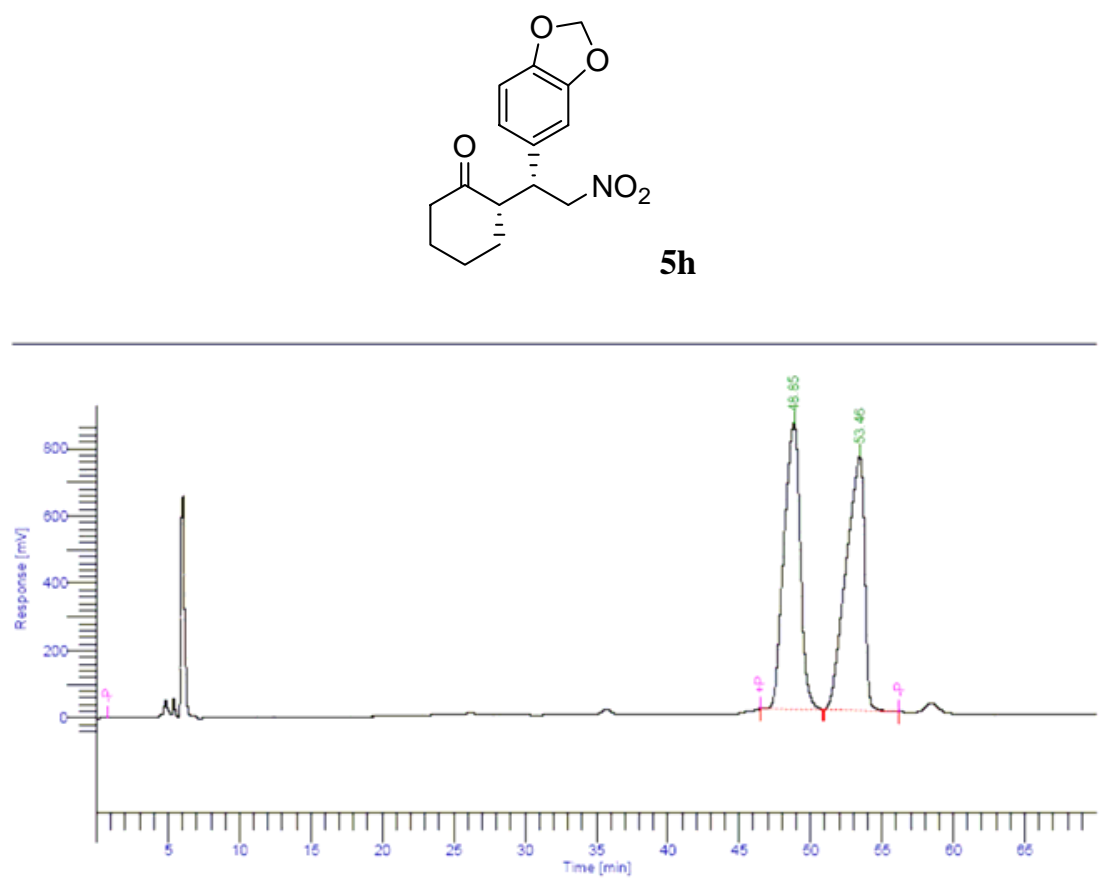

$\mathrm{ccl}$

\begin{tabular}{|c|c|c|c|c|c|}
\hline \multicolumn{6}{|c|}{ ccl-5-81-biao } \\
\hline $\begin{array}{c}\text { Peak } \\
\#\end{array}$ & $\begin{array}{l}\text { Time } \\
\text { [min] }\end{array}$ & $\begin{array}{c}\text { Area } \\
{\left[u v^{\prime} \text { sec] }\right.}\end{array}$ & $\begin{array}{l}\text { Height } \\
\text { [uV] }\end{array}$ & $\begin{array}{l}\text { Area } \\
{[\%]}\end{array}$ & $\begin{array}{c}\text { Norm, Area } \\
{[\%]}\end{array}$ \\
\hline \multirow{3}{*}{2} & 48.85 & $6.7568 \mathrm{e}+07$ & $8.484 \mathrm{e}+05$ & 49.81 & 49.81 \\
\hline & 53.46 & $6.8093 e+07$ & $7.550 \mathrm{e}+05$ & 50.19 & 50.19 \\
\hline & & $1.3566 e+08$ & $1.603 e+06$ & 100.00 & 100.00 \\
\hline
\end{tabular}

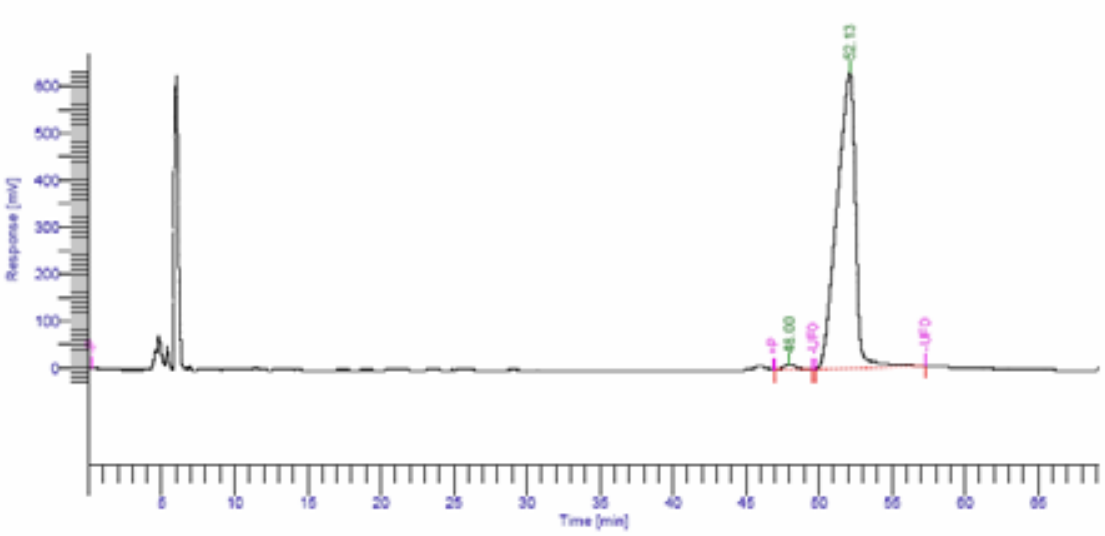

ccl

ccl-5-81-biao

\begin{tabular}{|c|c|c|c|c|c|}
\hline $\begin{array}{c}\text { Peak } \\
\#\end{array}$ & $\begin{array}{l}\text { Time } \\
\text { [min] }\end{array}$ & $\begin{array}{c}\text { Area } \\
{\left[u^{*} \text { 'sec] }\right.}\end{array}$ & $\begin{array}{l}\text { Height } \\
\text { [uv] }\end{array}$ & $\begin{array}{l}\text { Area } \\
{[\%]}\end{array}$ & $\begin{array}{c}\text { Norm, Area } \\
{[\%]}\end{array}$ \\
\hline & 48.00 & 676737.0806 & $1.041 \mathrm{e}+04$ & 1.20 & 1.20 \\
\hline & 52.13 & $5.5547 \mathrm{e}+07$ & $6.305 \mathrm{e}+05$ & 98.80 & 98.80 \\
\hline & & $5.6224 \mathrm{e}+07$ & $6.409 e+05$ & 100.00 & 100.00 \\
\hline
\end{tabular}


<smiles>COc1ccc([C@H](C[N+](=O)[O-])[C@H]2CCCCC2=O)cc1</smiles>

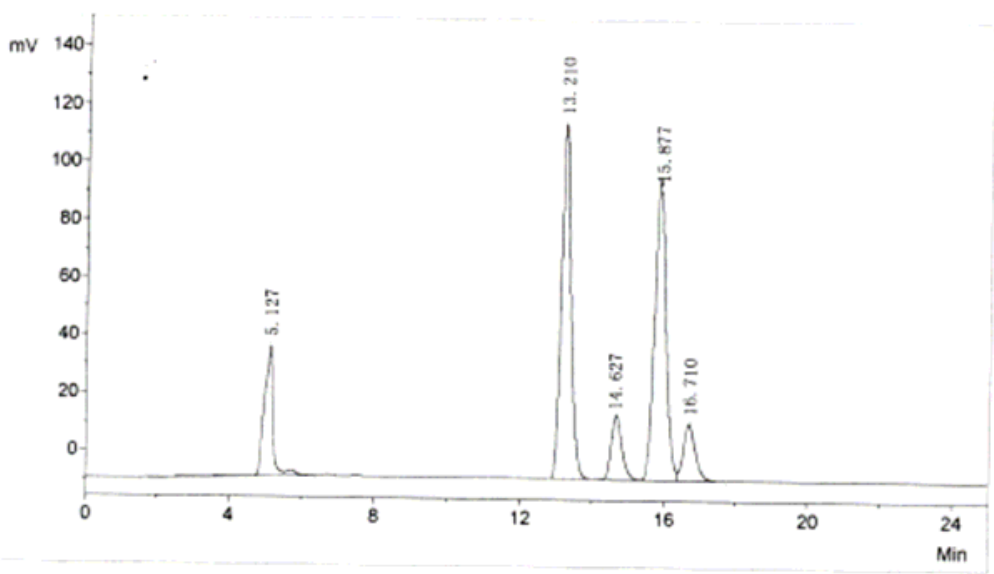

\begin{tabular}{rrrrrrr} 
No. PeakNo & ID. Name & R. Time & PeakHeight & \multicolumn{1}{c}{ PeakArea } & \multicolumn{1}{c}{ PerCent } \\
1 & 1 & Unknown & 5.127 & 45058.4 & 742648.2 & 11.9475 \\
2 & 2 & Unknown & 13.210 & 123005.5 & 2298414.4 & 36.9762 \\
3 & 3 & Unknown & 14.627 & 21073.9 & 450657.8 & 7.2500 \\
4 & 4 & Unknown & 15.877 & 100093.5 & 2277580.0 & 36.6410 \\
5 & 5 & Unknown & 16.710 & 19288.3 & 446636.9 & 7.1854 \\
Total & & & 308519.6 & 6215937.3 & 100.0000
\end{tabular}

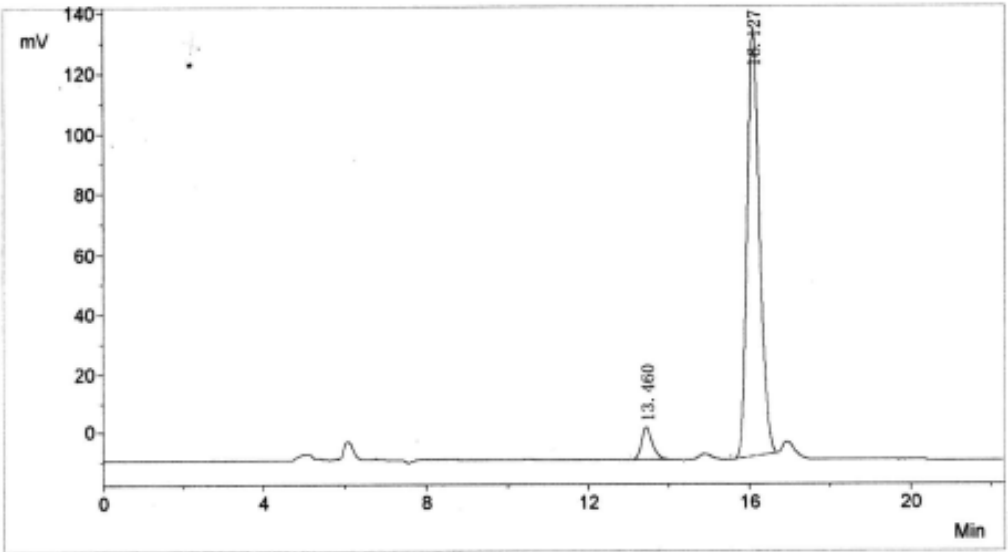

\begin{tabular}{cccrrrr} 
No. PeakNo & ID. Name & R. Time & PeakHeight & \multicolumn{1}{c}{ PeakArea } & \multicolumn{1}{c}{ PerCent } \\
\hline 1 & 1 & Unknown & 13.460 & 10298.3 & 197598.8 & 6.0314 \\
2 & 2 & Unknown & 16.127 & 141002.5 & 3078544.1 & 93.9686 \\
\hline Total & & & 151300.8 & 3276142.9 & 100.0000
\end{tabular}




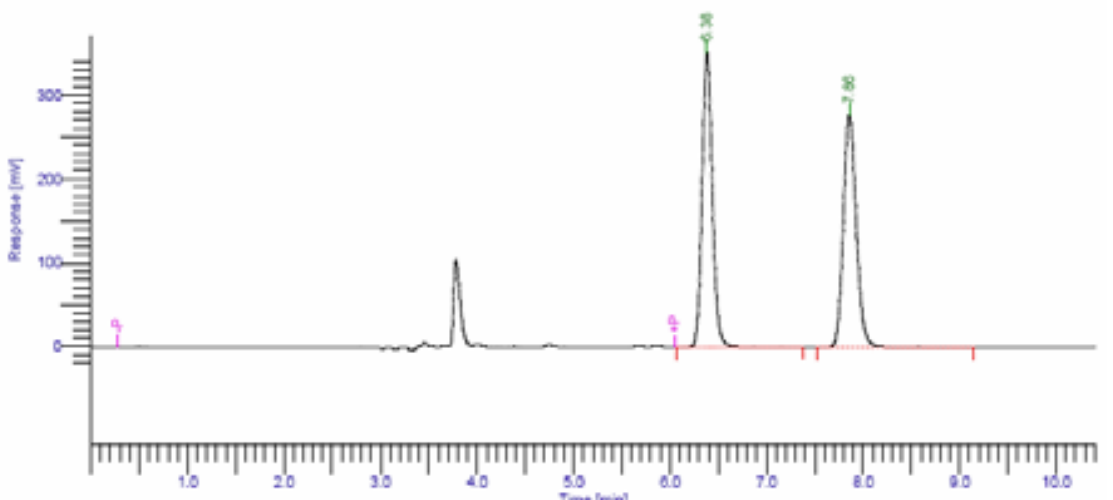

\section{$\mathrm{ccl}$}

ccl-5-900-race

\begin{tabular}{|c|c|c|c|c|c|}
\hline $\begin{array}{c}\text { Peak } \\
=\end{array}$ & $\begin{array}{l}\text { Time } \\
\text { [min] }\end{array}$ & $\begin{array}{c}\text { Area } \\
\text { [u'v'sec] }\end{array}$ & $\begin{array}{l}\text { Height } \\
\text { [uv] }\end{array}$ & $\begin{array}{c}\text { Area } \\
{[\%]}\end{array}$ & $\begin{array}{c}\text { Norm. Area } \\
{[\%]}\end{array}$ \\
\hline & 6.38 & $2.6235 \mathrm{e}+06$ & 3.511 & 50.06 & 50.06 \\
\hline & 7.86 & $2.6169 e+06$ & $2.775 e+05$ & 49.94 & 49.94 \\
\hline & & $5.2404 \mathrm{e}+06$ & $6.286 e+05$ & 100.00 & 100.00 \\
\hline
\end{tabular}

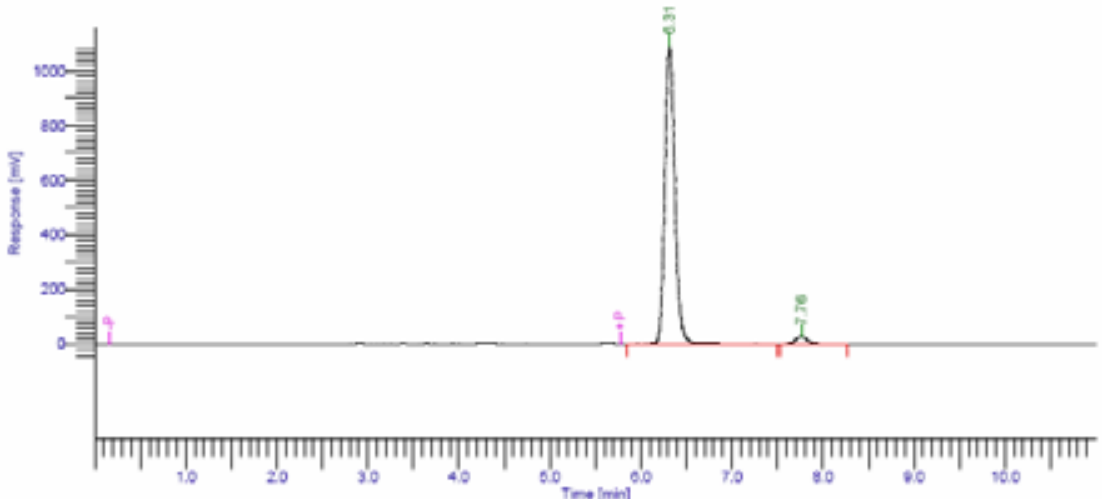

$\mathrm{ccl}$

ccl-5-90b-biac

\begin{tabular}{|c|c|c|c|c|c|}
\hline $\begin{array}{c}\text { Peak } \\
=\end{array}$ & $\begin{array}{l}\text { Time } \\
\text { [min] }\end{array}$ & $\begin{array}{c}\text { Area } \\
\text { [u'v'sec] }\end{array}$ & $\begin{array}{l}\text { Height } \\
\text { [uV] }\end{array}$ & $\begin{array}{c}\text { Area } \\
{[\%]}\end{array}$ & $\begin{array}{c}\text { Norm. Area } \\
{[\%]}\end{array}$ \\
\hline \multirow{3}{*}{2} & 6.31 & 8. $3790 \mathrm{e}+06$ & $1.089 \mathrm{e}+06$ & 97.00 & 97.00 \\
\hline & 7.76 & 259396.4899 & $2.919 \mathrm{e}+04$ & 3.00 & 3.00 \\
\hline & & $8.6304 \mathrm{e}+06$ & $1.110 \mathrm{e}+06$ & 100.00 & 100. \\
\hline
\end{tabular}



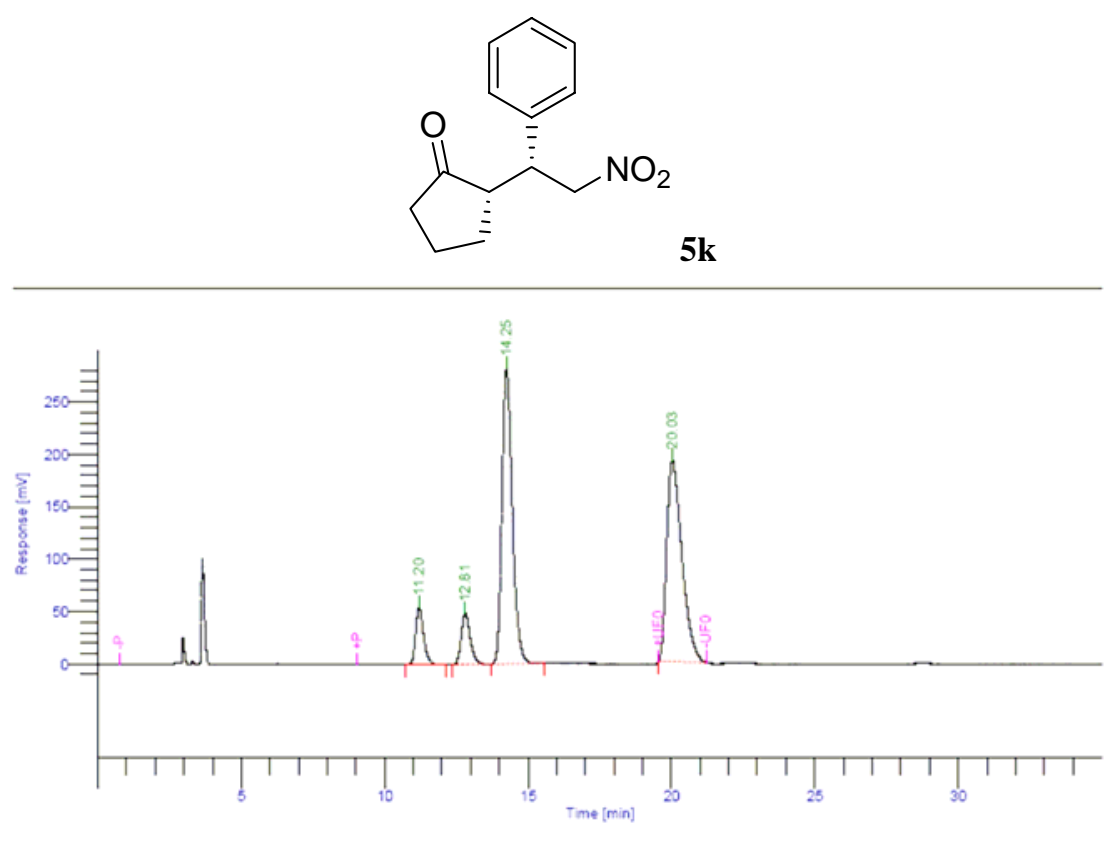

$\mathrm{ccl}$

\begin{tabular}{|c|c|c|c|c|c|}
\hline \multicolumn{6}{|c|}{ ccl-6-7a-race-biao } \\
\hline $\begin{array}{c}\text { Peak } \\
\#\end{array}$ & $\begin{array}{l}\text { Time } \\
\text { [min] }\end{array}$ & $\begin{array}{c}\text { Area } \\
\text { [uV*sec] }\end{array}$ & $\begin{array}{l}\text { Height } \\
\text { [uV] }\end{array}$ & $\begin{array}{l}\text { Area } \\
{[\%]}\end{array}$ & $\begin{array}{c}\text { Norm. Area } \\
{[\%]}\end{array}$ \\
\hline \multirow{5}{*}{$\begin{array}{l}1 \\
2 \\
3 \\
4\end{array}$} & 11.20 & $1.0576 e+06$ & $5.511 \mathrm{e}+04$ & 6.52 & 6.52 \\
\hline & 12.81 & $1.0600 \mathrm{e}+06$ & $4.879 \mathrm{e}+04$ & 6.54 & 6.54 \\
\hline & 14.25 & $7.0449 \mathrm{e}+06$ & $2.822 \mathrm{e}+05$ & 43.44 & 43.44 \\
\hline & 20.03 & $7.0564 \mathrm{e}+06$ & $1.918 \mathrm{e}+05$ & 43.51 & 43.51 \\
\hline & & $1.6219 e+07$ & $5.779 \mathrm{e}+05$ & 100.00 & 100. \\
\hline
\end{tabular}

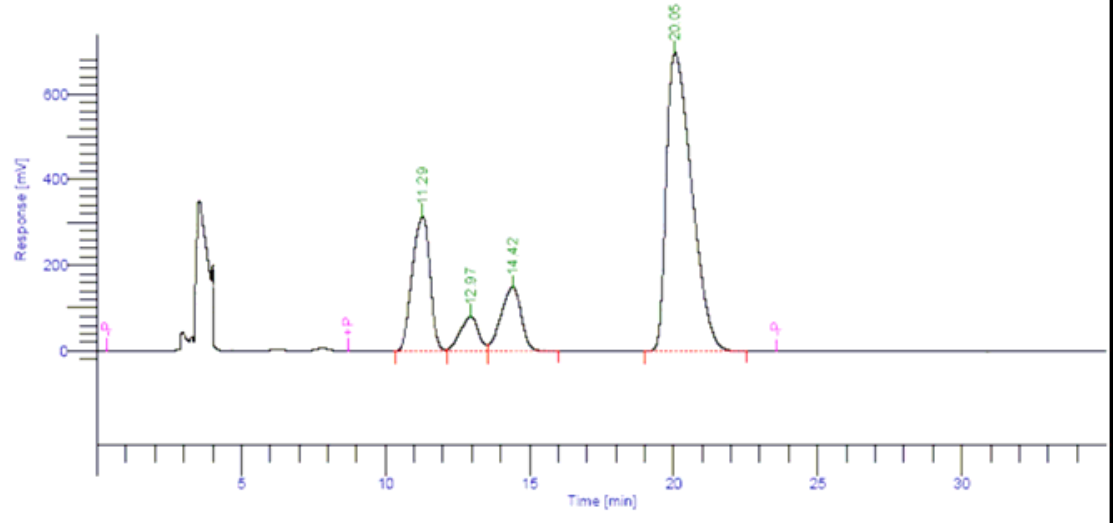

$\mathrm{ccl}$

ccl-6-7a-biao

\begin{tabular}{|c|c|c|c|c|c|}
\hline $\begin{array}{c}\text { Peak } \\
\#\end{array}$ & $\begin{array}{l}\text { Time } \\
\text { [min] }\end{array}$ & $\begin{array}{c}\text { Area } \\
{\left[\mathrm{uV}^{*} \mathrm{sec}\right]}\end{array}$ & $\begin{array}{l}\text { Height } \\
\text { [uV] }\end{array}$ & $\begin{array}{c}\text { Area } \\
{[\%]}\end{array}$ & $\begin{array}{c}\text { Norm, Area } \\
{[\%]}\end{array}$ \\
\hline 1 & \multirow{5}{*}{$\begin{array}{l}11.29 \\
12.97 \\
14.42 \\
20.05\end{array}$} & $1.3526 e+07$ & $3.149 \mathrm{e}+05$ & 20.23 & 20.23 \\
\hline 2 & & $3.5609 \mathrm{e}+06$ & $8.061 \mathrm{e}+04$ & 5.33 & 5.33 \\
\hline 3 & & $7.2435 e+06$ & $1.493 e+05$ & 10.84 & 10.84 \\
\hline 4 & & $4.2519 e+07$ & $6.973 e+05$ & 63.60 & 63.60 \\
\hline & & $849 e+07$ & 42 & 0 & 100 \\
\hline
\end{tabular}


<smiles>CC(=O)CC(CN(O)O[Na])c1ccccc1</smiles>

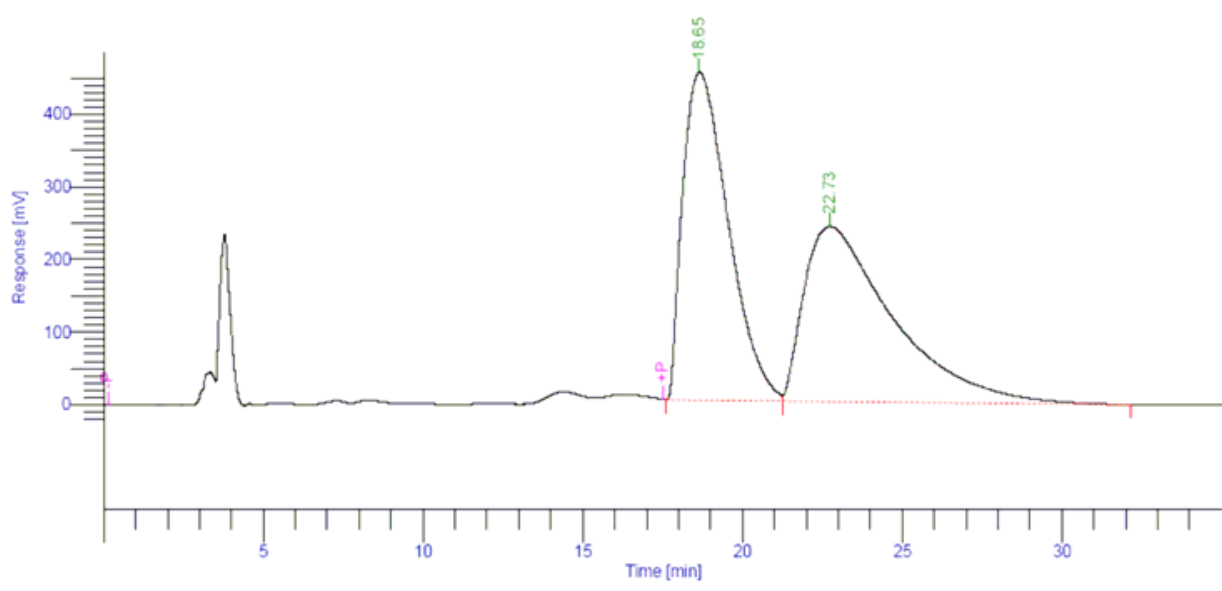

$\mathrm{ccl}$

ccl-5-86-race-biao

\begin{tabular}{|c|c|c|c|c|c|}
\hline $\begin{array}{c}\text { Peak } \\
\#\end{array}$ & $\begin{array}{l}\text { Time } \\
\text { [min] }\end{array}$ & $\begin{array}{c}\text { Area } \\
{\left[\mathrm{uV}^{*} \mathrm{sec}\right]}\end{array}$ & $\begin{array}{l}\text { Height } \\
\text { [uV] }\end{array}$ & $\begin{array}{l}\text { Area } \\
{[\%]}\end{array}$ & $\begin{array}{c}\text { Norm. Area } \\
{[\%]}\end{array}$ \\
\hline 1 & 18.65 & $4.4634 \mathrm{e}+07$ & $4.515 e+05$ & 49.06 & 49.06 \\
\hline 2 & 22.73 & $4.6349 e+07$ & $2.411 e+05$ & 50.94 & 50.94 \\
\hline & & $9.0983 e+07$ & $6.926 e+05$ & 100.00 & 100.00 \\
\hline
\end{tabular}

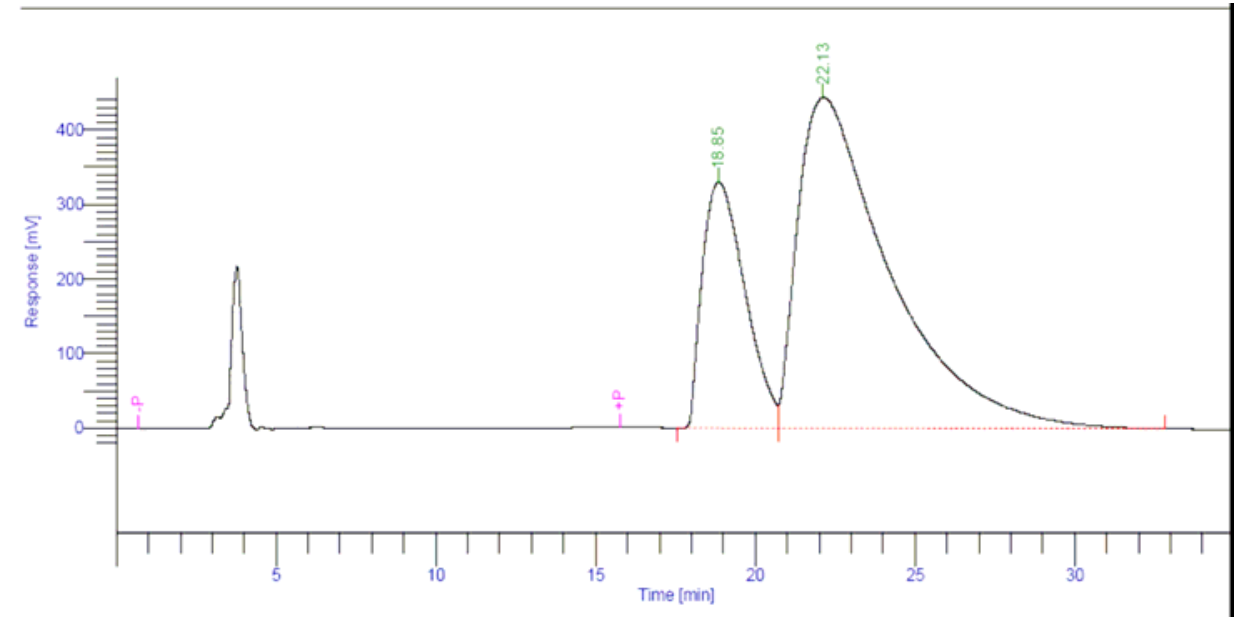

$\mathrm{ccl}$

ccl-5-86-biao

\begin{tabular}{|c|c|c|c|c|c|}
\hline $\begin{array}{c}\text { Peak } \\
\#\end{array}$ & $\begin{array}{l}\text { Time } \\
\text { [min] }\end{array}$ & $\begin{array}{c}\text { Area } \\
{\left[\mathrm{uV}^{*} \mathrm{sec}\right]}\end{array}$ & $\begin{array}{l}\text { Height } \\
\text { [uV] }\end{array}$ & $\begin{array}{c}\text { Area } \\
{[\%]}\end{array}$ & $\begin{array}{c}\text { Norm. Area } \\
{[\%]}\end{array}$ \\
\hline & 18.85 & $3.0622 e+07$ & $3.302 \mathrm{e}+05$ & 25,66 & 25.66 \\
\hline & 22.13 & $8,8702 e+07$ & $4.434 e+05$ & 74.34 & 74.34 \\
\hline & & $1.1932 \theta+08$ & $7.735 e+05$ & 100.0 & 100.00 \\
\hline
\end{tabular}


<smiles>CC(=O)[C@H](C)[C@H](C[N+](=O)[O-])c1ccccc1</smiles><smiles>O=C(C[18OH])C[C@H](C[N+](=O)[O-])c1ccccc1</smiles>

$5 \mathrm{~m}$

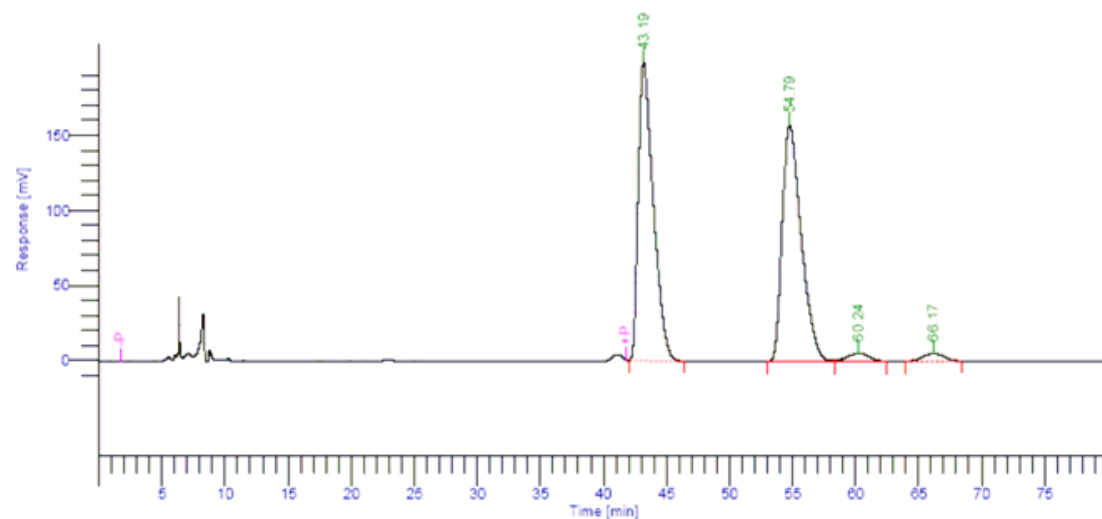

$\mathrm{ccl}$

\begin{tabular}{|c|c|c|c|c|c|}
\hline \multicolumn{6}{|c|}{ ccl-6-84b-race-biao-qiandianxiao-houdianda } \\
\hline $\begin{array}{c}\text { Peak } \\
\#\end{array}$ & $\begin{array}{l}\text { Time } \\
\text { [min] }\end{array}$ & $\begin{array}{c}\text { Area } \\
\text { [uv*sec] }\end{array}$ & $\begin{array}{l}\text { Height } \\
\text { [uV] }\end{array}$ & $\begin{array}{l}\text { Area } \\
{[\%]}\end{array}$ & $\begin{array}{c}\text { Norm. Area } \\
{[\%]}\end{array}$ \\
\hline \multirow[t]{5}{*}{1} & \multirow{5}{*}{$\begin{array}{l}43.19 \\
54.79 \\
60.24 \\
66.17\end{array}$} & $1.6459 e+07$ & $1.993 e+05$ & 48.02 & 48.02 \\
\hline & & $1.6553 e+07$ & $1.576 \mathrm{e}+05$ & 48.30 & 48.30 \\
\hline & & 635720.9397 & 5566.9825 & 1.85 & 1.85 \\
\hline & & 624451.5423 & 5387.0975 & 1.82 & 1.82 \\
\hline & & $3.4271 \mathrm{e}+07$ & $3.679 e+05$ & 100.00 & 100.00 \\
\hline
\end{tabular}

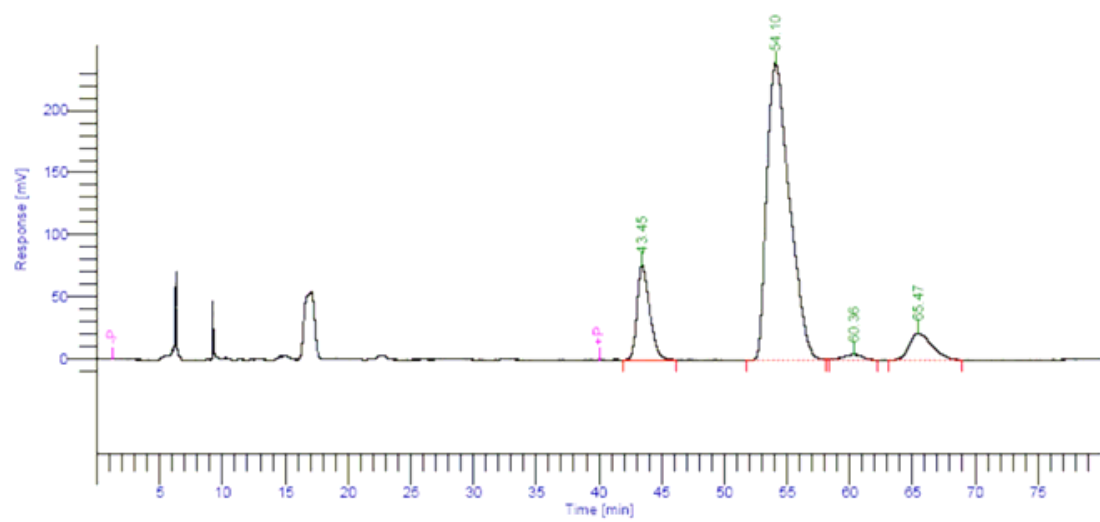

$\mathrm{ccl}$

ccl-6-84b-biao-qiandianxiao-houdianda

\begin{tabular}{|c|c|c|c|c|c|}
\hline $\begin{array}{c}\text { Peak } \\
\#\end{array}$ & $\begin{array}{l}\text { Time } \\
\text { [min] }\end{array}$ & $\begin{array}{c}\text { Area } \\
{\left[\mathrm{uV}^{*} \mathrm{sec}\right]}\end{array}$ & $\begin{array}{l}\text { Height } \\
\text { [uV] }\end{array}$ & $\begin{array}{l}\text { Area } \\
{[\%]}\end{array}$ & $\begin{array}{c}\text { Norm. Area } \\
{[\%]}\end{array}$ \\
\hline 1 & \multirow{5}{*}{$\begin{array}{l}43.45 \\
54.10 \\
60.36 \\
65.47\end{array}$} & $5.2952 e+06$ & $7.642 \mathrm{e}+04$ & 13.70 & 13.70 \\
\hline 2 & & $3.0168 \mathrm{e}+07$ & $2.392 \mathrm{e}+05$ & 78.04 & 78.04 \\
\hline 3 & & 459152.1056 & 4211.3901 & 1.19 & 1.19 \\
\hline 4 & & $2.7349 \mathrm{e}+06$ & $2.181 e+04$ & 7.07 & 7.07 \\
\hline & & $3.8657 e+07$ & $3.417 e+05$ & 100.00 & 100.00 \\
\hline
\end{tabular}



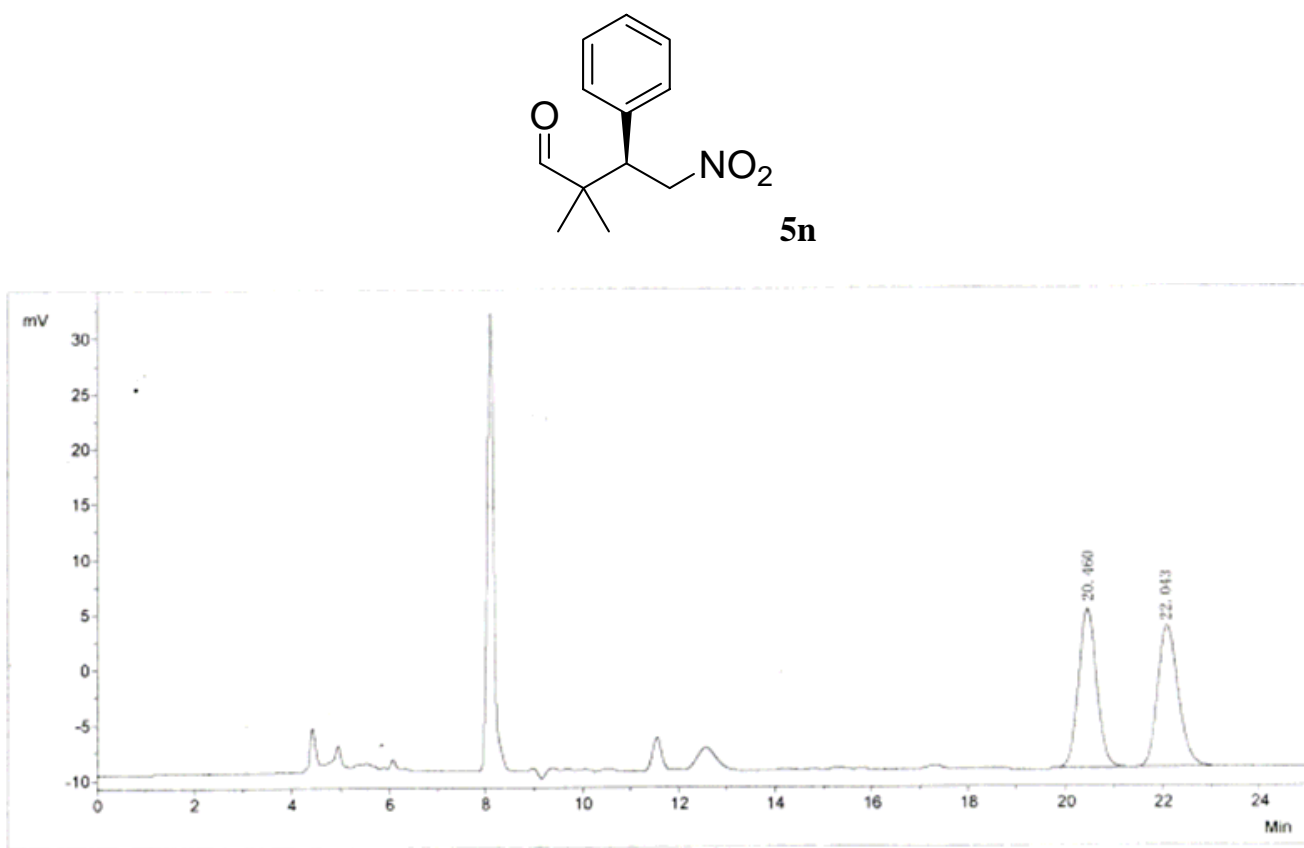

\begin{tabular}{rrrrr} 
No. & R. Time & PeakHeight & PeakArea & PerCent \\
\hline 1 & 20.160 & 14223.0 & 371065.2 & 49.9135 \\
2 & 22.043 & 12401.0 & 372350.6 & 50.0865 \\
\hline Total & & 26624.0 & 743415.8 & 100.0000
\end{tabular}

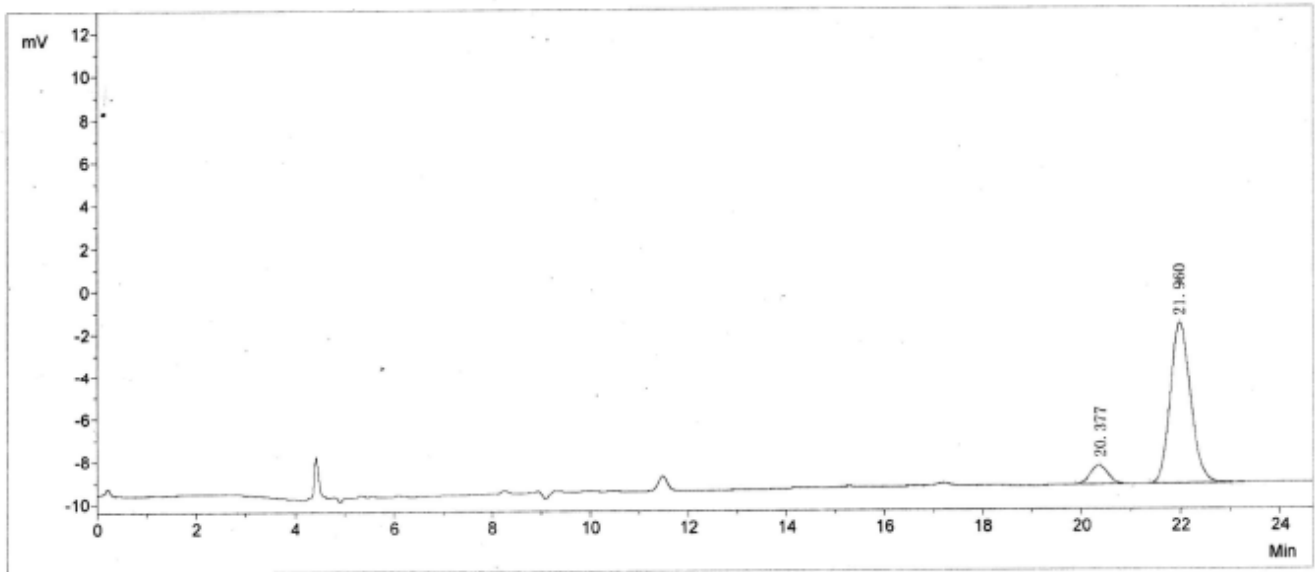

\begin{tabular}{rrrrr} 
No. & R. Time & PeakHeight & PeakArea & \multicolumn{1}{c}{ PerCent } \\
1 & 20.377 & 871.4 & 21817.1 & 9.1964 \\
2 & 21.960 & 7529.3 & 215418.2 & 90.8036 \\
\hline Total & 8400.7 & 237235.3 & 100.0000
\end{tabular}



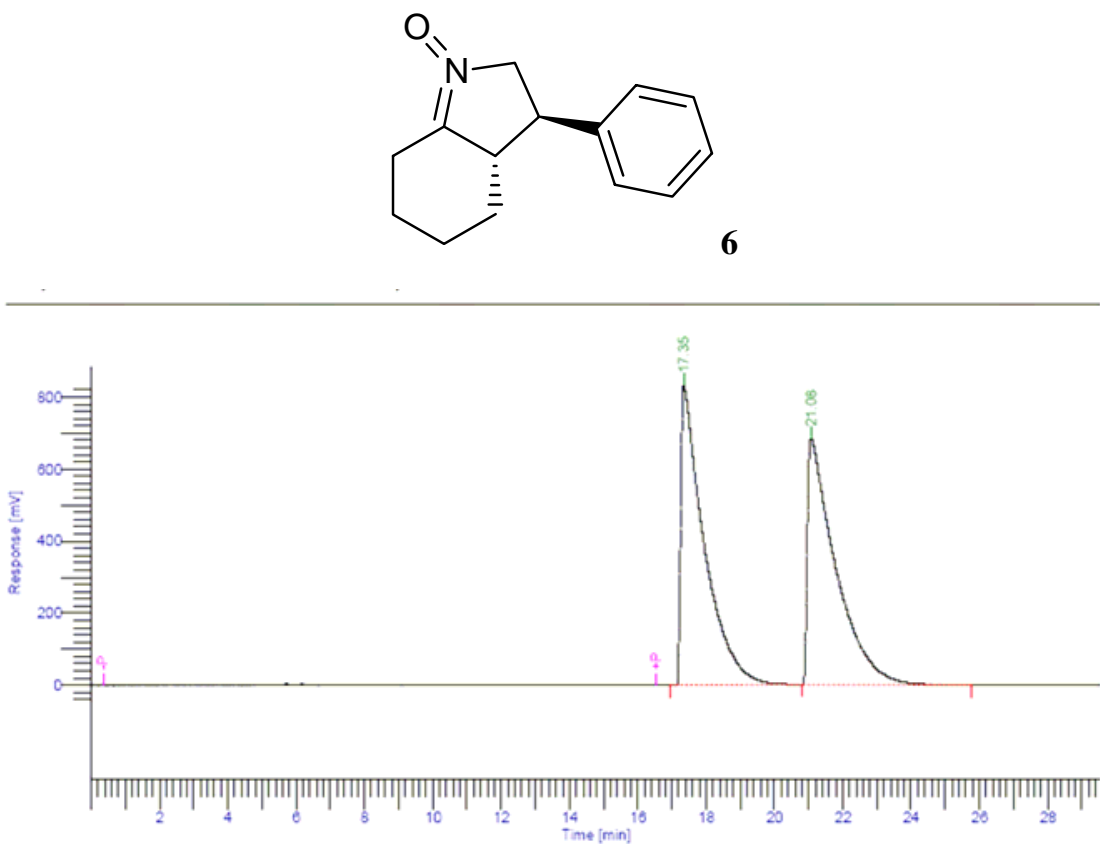

$\mathrm{ccl}$

ccl-6-29-race-biao

\begin{tabular}{|c|c|c|c|c|c|}
\hline $\begin{array}{l}\text { Peak } \\
\#\end{array}$ & $\begin{array}{l}\text { Time } \\
\text { [min] }\end{array}$ & $\begin{array}{c}\text { Area } \\
{\left[\mathrm{uV}^{*} \mathrm{sec}\right]}\end{array}$ & $\begin{array}{l}\text { Height } \\
\text { [uV] }\end{array}$ & $\begin{array}{l}\text { Area } \\
{[\%]}\end{array}$ & $\begin{array}{c}\text { Norm. Area } \\
{[\%]}\end{array}$ \\
\hline 1 & \multirow{3}{*}{$\begin{array}{l}17.35 \\
21.08\end{array}$} & 3.91 & 8.3. & 50.08 & \\
\hline 2 & & 3.900 & $6.841 e+05$ & 49.92 & 49. \\
\hline & & .8133 & $1.518 e+06$ & 100.00 & 100 \\
\hline
\end{tabular}

Result File

Sequence File : D:HPLCicclivend-1, seq

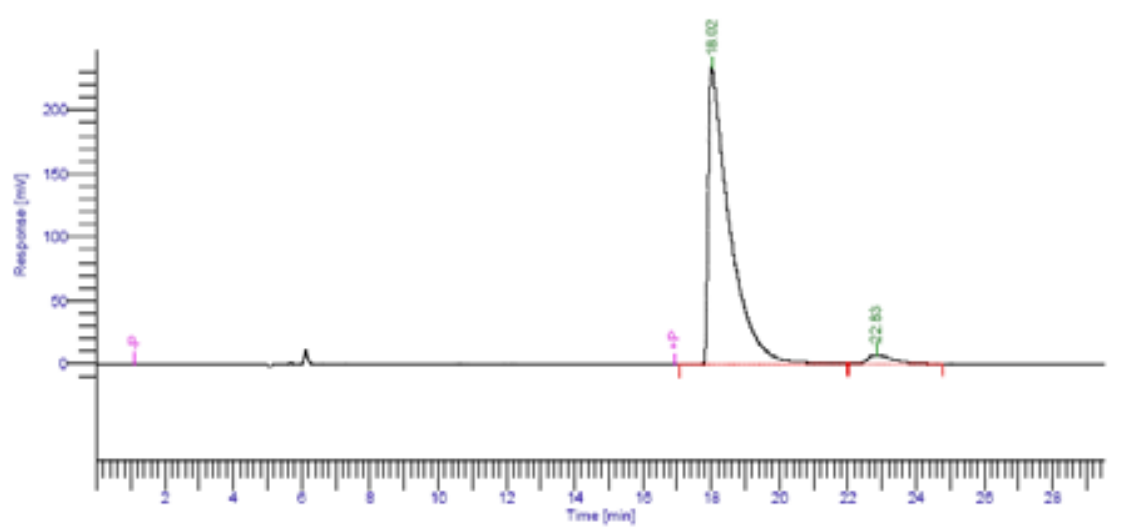

$\mathrm{ccl}$

ccl-6-30-biao

\begin{tabular}{|c|c|c|c|c|c|}
\hline $\begin{array}{c}\text { Peak } \\
\#\end{array}$ & $\begin{array}{l}\text { Time } \\
\text { [min] }\end{array}$ & $\begin{array}{c}\text { Area } \\
\text { [u'v"sec] }\end{array}$ & $\begin{array}{l}\text { Height } \\
\text { [uV] }\end{array}$ & $\begin{array}{c}\text { Area } \\
{[\%]}\end{array}$ & $\begin{array}{c}\text { Norm. Area } \\
{[\%]}\end{array}$ \\
\hline & 18.02 & $1.0250 \mathrm{e}+07$ & $2.342 e+05$ & 96.06 & 96.06 \\
\hline & 22.83 & 419961.8226 & 7652.7734 & 394 & 3.94 \\
\hline & & 1.0 & $2.418 \mathrm{e}+05$ & 0.00 & 100 \\
\hline
\end{tabular}

